\title{
Three-Component Aminoselenation of Arynes
}

\author{
Rahul N. Gaykar, Avishek Guin, Subrata Bhattacharjee, and Akkattu T. Biju* \\ Department of Organic Chemistry, Indian Institute of Science, Bangalore-560012, \\ India \\ atbiju@iisc.ac.in
}

\section{Supporting Information}

$\begin{array}{ll}\text { 1. General Information } & \text { S02 }\end{array}$

2. General Procedure for the Synthesis of Aniline Derivatives $\quad$ S03

3. General Procedure for the Synthesis of Diselenides $\quad$ S03

4. General Procedure for the Optimization of the Reaction Conditions $\quad$ S04

5. General Procedure for the Aminoselenation of Arynes $\quad$ S04

6. Procedure for the Synthesis of $N$-methyl- $N$-phenyl-2-(phenylthio)aniline S06

$\begin{array}{ll}\text { 7. Mechanistic Experiments } & \text { S07 }\end{array}$

$\begin{array}{ll}\text { 8. X-ray Data of } \mathbf{4 f} \text { and } \mathbf{1 3} & \text { S07 }\end{array}$

9. Synthesis and Characterization of 2-Selanyl Aniline Derivatives $\quad$ S11

10. ${ }^{1} \mathrm{H}$ and ${ }^{13} \mathrm{C}$ NMR Spectra of 2-Selanyl Aniline Derivatives $\quad \mathrm{S} 31$ 


\section{General Information}

Unless otherwise specified, all reactions were carried out under an atmosphere of argon in flame-dried reaction vessels with Teflon screw caps. $25{ }^{\circ} \mathrm{C}$ Corresponds to the room temperature (rt) of the lab when the experiments were carried out. Dry $\mathrm{CH}_{3} \mathrm{CN}$ was purchased from commercial sources and was stored under argon over $4 \AA$ molecular sieves. CsF was dried by heating at $110{ }^{\circ} \mathrm{C}$ for $12 \mathrm{~h}$ and left to cool under argon. The 2-(trimethylsilyl)phenyl trifluoromethanesulfonate $\mathbf{2 a}$ and the other symmetric and unsymmetrical aryne precursors were synthesized following literature procedure. ${ }^{1}$

Analytical thin layer chromatography was performed on TLC Silica gel $60 \mathrm{~F}_{254}$. Visualization was accomplished with short wave UV light or $\mathrm{KMnO}_{4}$ staining solutions followed by heating. Flash chromatography was performed on silica gel (230-400 mesh) by standard techniques eluting with Pet. Ether- $\mathrm{CH}_{2} \mathrm{Cl}_{2}$ solvent system.

All compounds were fully characterized. ${ }^{1} \mathrm{H}$ and ${ }^{13} \mathrm{C}$ NMR spectra were recorded on Bruker Ultrashield spectrometer or Jeol ECZ spectrometer in $\mathrm{CDCl}_{3}$ as solvent. Chemical shifts ( $\delta)$ are given in ppm. The residual solvent signals were used as references and the chemical shifts converted to the TMS scale $\left(\mathrm{CDCl}_{3}: \delta_{\mathrm{H}}=7.26 \mathrm{ppm}, \delta_{\mathrm{C}}=77.16 \mathrm{ppm}\right)$. Infrared (FT-IR) spectra were recorded on a Perkin Elmer Spectrum BX spectrophotometer, $v$-max in $\mathrm{cm}^{-1}$. HRMS (ESI) data were recorded on a Waters Xevo G2-XS Q-TOF instrument.

\footnotetext{
${ }^{1}$ (a) Sato, Y.; Tamura, T.; Kinbara, A.; Morib, M Adv. Synth. Catal. 2007, 349, 647. (b) Peña, D.; Cobas,
} A.; Pérez, D.; Guitián, E. Synthesis, 2002, 1454. 


\section{General Procedure for the Synthesis of Aniline Derivatives ${ }^{2}$}

The tertiary amines derivatives $2 \mathbf{a}, \mathbf{2 f}, \mathbf{2 p}$ and $7 \mathbf{a}$ were purchased from commercial sources and used as received without further purification. Substrates $\mathbf{2 b - e , ~} \mathbf{2 g - 0}$ and $7 \mathbf{c}$ were prepared using modified procedure ( $2 c$ as an example below). ${ }^{2}$

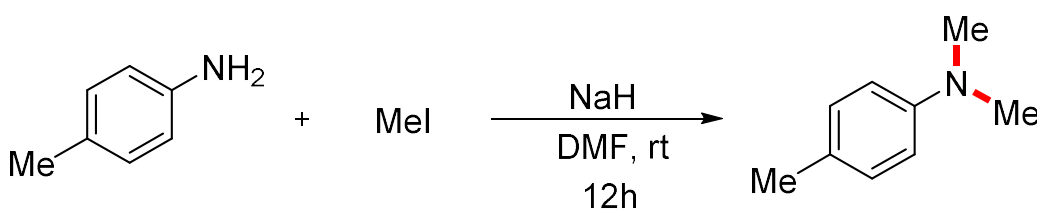

To a suspension of $\mathrm{NaH}(1.1 \mathrm{~g}, 60 \%$ dispersion in mineral oil, $28 \mathrm{mmol})$ in DMF $(15 \mathrm{~mL})$ at $0{ }^{\circ} \mathrm{C}$ was added an $p$-toluidine $(1.0 \mathrm{~g}, 9.0 \mathrm{mmol})$ followed by the subsequent addition of methyl iodide $(28.0 \mathrm{mmol})$ under argon, and the reaction mixture was stirred for $12 \mathrm{~h}$ at $25{ }^{\circ} \mathrm{C}$. The reaction was quenched with water, and organic materials were extracted with EtOAc three times. The combined extracts were washed with brine and dried over $\mathrm{Na}_{2} \mathrm{SO}_{4}$. After removal of the solvent under reduced pressure, the residue was purified by column chromatography (eluent: EtOAc: petroleum ether $=5: 95)$ to afford the tertiary amine derivative $2 \mathrm{c}(0.830 \mathrm{~g}, 66 \%)$.

\section{General Procedure for the Synthesis of Diselenides ${ }^{3}$}<smiles>Ic1c[R1]ccc1I</smiles>

$$
\begin{aligned}
& \underset{2.0 \text { equiv Se, } \mathrm{KOH} \text { (2.0 equiv) }}{\stackrel{\text { CuO nanoparticles ( } 10 \mathrm{~mol} \%)}{\longrightarrow}} \\
& \text { DMSO, } 90^{\circ} \mathrm{C}
\end{aligned}
$$

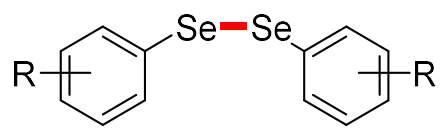

To a stirred solution of Se metal $(2.0 \mathrm{mmol})$ and aryl iodide $(1.0 \mathrm{mmol})$ in dry DMSO (2.0 $\mathrm{mL}$ ) was added $\mathrm{CuO}$ nanoparticles $(10.0 \mathrm{~mol} \%$ ) followed by $\mathrm{KOH}$ (2.0 equiv) under argon atmosphere at $90{ }^{\circ} \mathrm{C}$. The progress of the reaction was monitored by TLC. After the reaction was complete, the reaction mixture was allowed to cool to room temperature and it was then quenched with water and extracted with EtOAc. The combined organic layers were dried over anhydrous $\mathrm{Na}_{2} \mathrm{SO}_{4}$. The solvent was removed under reduced pressure, and the residue was

\footnotetext{
${ }^{2}$ Lv, Y.; Zheng, Y.; Li, Y.; Xiong, T.; Zhang, J.; Liu, Q.; Zhang, Q. Chem. Commun. 2013, 49, 8866.

${ }^{3}$ Singh, D.; Deobald, A. M.; Camargo, L. R. S.; Tabarelli, G.; Rodrigues, O. E. D.; Braga, A. L. Org. Lett. 2010, 12, 3288.
} 
purified by flash chromatography on a silica gel column chromatography (Pet Ether) to give the pure diselenides.

\section{General Procedure for the Optimization of Reaction Conditions}<smiles>CCOc1ccccc1C</smiles>

$1 \mathrm{a}$<smiles>CN(C)c1ccccc1</smiles>

3a

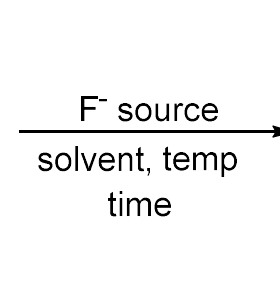<smiles>C=C(/C=C\c1ccccc1)N(C)c1ccccc1[Se]c1ccccc1</smiles>

$4 a$<smiles>CN(c1ccccc1)c1ccccc1</smiles>

$5 a$

To a flame-dried screw-capped test tube equipped with a magnetic stir bar was added the fluoride source $(0.60 \mathrm{mmol})$ inside the glove-box. Phenylselenyl bromide 3a $(0.25 \mathrm{mmol})$ was added and the mixture was dissolved in $1.0 \mathrm{~mL}$ of THF outside the glove-box under argon and to this stirring solution was added $N, N$-dimethyl aniline $\mathbf{2 a}(0.030 \mathrm{~g}, 32 \mu \mathrm{L}, 0.25 \mathrm{mmol})$ and 2(trimethylsilyl)phenyl trifluoromethanesulfonate $1 \mathrm{a}(0.090 \mathrm{~g}, 73 \mu \mathrm{L}, 0.30 \mathrm{mmol})$ at $25^{\circ} \mathrm{C}$. Then the reaction mixture was allowed to react at indicated temperature and time. After indicated time over, the reaction was stopped, the solvent was evaporated and the crude residue pre-adsorbed on silica gel and purified by flash column chromatography on silica gel to afford the corresponding 2-selanyl aniline derivatives 4 .

Table S1: Optimization of the Reaction Conditions ${ }^{\mathrm{a}}$

\begin{tabular}{|c|l|l|c|c|c|c|}
\hline entry & \multicolumn{1}{|c|}{ F source } & solvent & $\begin{array}{c}\text { Temp } \\
\left({ }^{\circ} \mathrm{C}\right)\end{array}$ & $\begin{array}{c}\text { Time } \\
(\mathrm{h})\end{array}$ & $\begin{array}{c}\text { Yield of 4a } \\
(\%)^{\mathrm{b}}\end{array}$ & $\begin{array}{c}\text { Yield of 5 } \\
(\%)^{\mathrm{b}}\end{array}$ \\
\hline 1 & $\mathrm{KF} / 18$-crown-6 & THF & 25 & 12 & 55 & 18 \\
\hline $2^{\mathrm{c}}$ & $\mathrm{KF} / 18$-crown-6 & THF & 25 & 12 & 36 & 20 \\
\hline $3^{\mathrm{d}}$ & $\mathrm{KF} / 18$-crown-6 & THF & 25 & 12 & 50 & 24 \\
\hline 4 & $\mathrm{KF} / 18$-crown-6 & DME & 25 & 12 & 48 & 16 \\
\hline 5 & $\mathrm{CsF}$ & $\mathrm{CH}_{3} \mathrm{CN}$ & 25 & 12 & $<5$ & $<5$ \\
\hline 6 & $\mathrm{TBAF}$ & THF & 25 & 12 & $<5$ & $<5$ \\
\hline 7 & $\mathrm{TBAT}$ & toluene & 25 & 12 & $<5$ & $<5$ \\
\hline 8 & $\mathrm{KF} / 18$-crown-6 & THF & $-10-25$ & 12 & 27 & 29 \\
\hline 9 & $\mathrm{KF} / 18$-crown-6 & THF & 60 & 12 & 16 & 38 \\
\hline $10^{\mathrm{e}}$ & $\mathrm{KF} / 18$-crown-6 & THF & 25 & 12 & 51 & $<5$ \\
\hline $11^{\mathrm{f}}$ & $\mathrm{KF} / 18$-crown-6 & THF & 25 & 12 & 72 & $<5$ \\
\hline $13^{\mathrm{g}}$ & $\mathrm{KF} / 18$-crown-6 & THF & 25 & 24 & 72 & $<5$ \\
\hline
\end{tabular}


a Standard conditions: 1a $(0.30 \mathrm{mmol}), \mathbf{2 a}(0.25 \mathrm{mmol}), \mathbf{3 a}(0.25 \mathrm{mmol})$, fluoride source (2.4 equiv), solvent $(1.0 \mathrm{~mL}), 25^{\circ} \mathrm{C}$ and $12 \mathrm{~h}$. ${ }^{\mathrm{b}}$ Yield of isolated product(s) is given. ${ }^{\mathrm{c}} \mathrm{PhSeCl}(0.25 \mathrm{mmol})$ was used instead of PhSeBr. ${ }^{d} \mathrm{PhSeSPh}(0.375 \mathrm{mmol})$ was used instead of $\mathrm{PhSeBr}$ and $0.5 \mathrm{mmol}$ of $1 \mathrm{a}$, fluoride source (4.0 equiv) was used, ${ }^{\mathrm{e}} 1.5$ eqiv. of $\mathbf{2 a}$ was used. ${ }^{\mathrm{f}} 2.0$ equiv of $\mathbf{1 a}, 1.5$ equiv of $\mathbf{2 a}$, fluoride source (4.0 equiv) was used. ${ }^{\mathrm{g}}$ Reaction was stirred for $24 \mathrm{~h}$

\section{General Procedure for the Aminoselenation of Arynes}

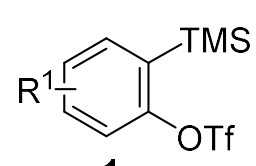

1<smiles>CN(C)c1ccc([As])cc1</smiles>

3

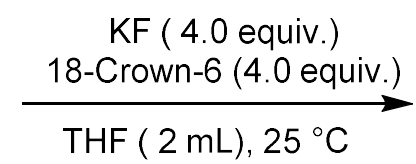

$12 \mathrm{~h}$<smiles>[R]c1ccccc1N(C)c1ccccc1Sc1ccccc1</smiles>

$$
\mathrm{Y}=\mathrm{Br}, \mathrm{PhSe}
$$

To a flame-dried screw-capped test tube equipped with a magnetic stir bar was added the 18crown-6 (0.528 g, $2.0 \mathrm{mmol}), \mathrm{KF}(0.116 \mathrm{~g}, 2.0 \mathrm{mmol})$ inside the glove-box. Electrophilic selenium source $3(0.75 \mathrm{mmol})$ was added and the mixture was dissolved in $2.0 \mathrm{~mL}$ of THF outside the glove box under argon and kept stirring for five minutes. To this stirring mixture was added $0.5 \mathrm{mmol}$ of the $N, N$-dimethyl aniline derivatives 2 and aryne precursor 1 (1.0 mmol). Then the reaction mixture was allowed to react at $25{ }^{\circ} \mathrm{C}$ for $12 \mathrm{~h}$. After $12 \mathrm{~h}$, the reaction was stopped, the solvent was evaporated and the crude residue pre-adsorbed on silica gel and purified by flash column chromatography on silica gel to afford the corresponding 2-selanyl aniline derivatives 4 in moderate to good yield.

\section{Procedure for the $1.0 \mathrm{mmol}$ scale}
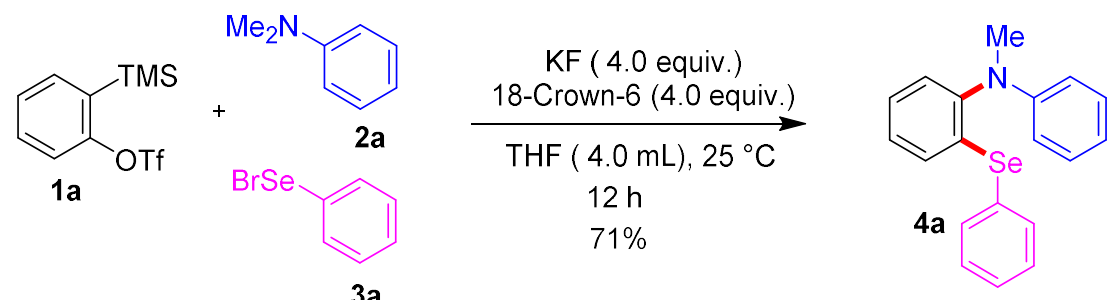

3a

To a flame-dried screw-capped test tube equipped with a magnetic stir bar was added the 18crown-6 (1.056 g, $4.0 \mathrm{mmol}), \mathrm{KF}(0.232 \mathrm{~g}, 4.0 \mathrm{mmol})$ inside the glove-box. Phenylselenyl 
bromide 3a (0.354 g, $1.5 \mathrm{mmol})$ was added and the mixture was dissolved in $4.0 \mathrm{~mL}$ of THF outside the glove-box under argon and kept stirring for five minutes. To this stirring mixture was added $N, N$-dimethyl aniline $\quad \mathbf{2 a}(0.121 \quad \mathrm{~g}, \quad 127 \mu \mathrm{L}, \quad 1.0 \quad \mathrm{mmol})$ and 2(trimethylsilyl)phenyltrifluoromethanesulfonate $1 \mathrm{a}(0.597 \mathrm{~g}, 486 \mu \mathrm{L}, 2.0 \mathrm{mmol})$. Then the reaction mixture was allowed to react at $25{ }^{\circ} \mathrm{C}$ for $12 \mathrm{~h}$. After $12 \mathrm{~h}$, the reaction was stopped, the solvent was evaporated and the crude residue pre-adsorbed on silica gel and purified by flash column chromatography (Pet.ether/DCM $=95 / 05$ ) on silica gel to afforded $N$-methyl- $N$-phenyl2-(phenylselanyl)aniline $4 \mathbf{a}$ as a white solid (0.240 g, 71\% yield).

\section{Procedure for the Preparation of $N$-methyl- $N$-phenyl-2- (phenylthio)aniline ${ }^{4}$}

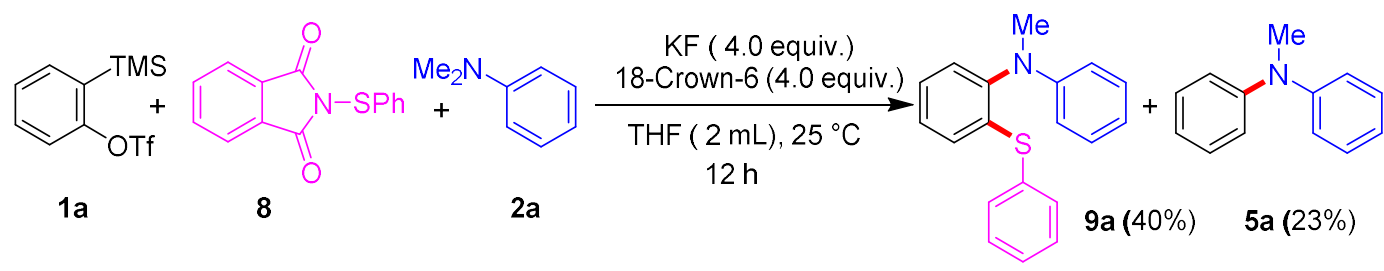

To a flame-dried screw-capped test tube equipped with a magnetic stir bar was added the 18-crown-6 (0.264 g, $1.0 \mathrm{mmol}), \mathrm{KF}(0.058 \mathrm{~g}, 1.0 \mathrm{mmol})$ inside the glove-box. 2(phenylthio)isoindoline-1,3-dione $8(0.096 \mathrm{~g}, 0.375 \mathrm{mmol})$ was added and the mixture was dissolved in $1.0 \mathrm{~mL}$ of THF outside the glove-box under argon and kept stirring for five minutes. To this stirring mixture was added $N, N$-dimethylaniline $2 \mathrm{a}(0.030 \mathrm{~g}, 32 \mu \mathrm{L}, 0.25 \mathrm{mmol})$ and 2(trimethylsilyl)phenyltrifluoromethanesulfonate $1 \mathrm{a}(0.149 \mathrm{~g}, 121 \mu \mathrm{L}, 0.5 \mathrm{mmol})$. Then the reaction mixture was allowed to react at $25{ }^{\circ} \mathrm{C}$ for $12 \mathrm{~h}$. After $12 \mathrm{~h}$, the reaction was stopped, the solvent was evaporated and the crude residue pre-adsorbed on silica gel and purified by flash column chromatography on silica gel to afford the $N$-methyl- $N$-phenyl-2-(phenylthio)aniline 9a in $40 \%$ yield along with $N$-arylated product $\mathbf{5 a}$ in $23 \%$ yield.

\footnotetext{
${ }^{4}$ Gaykar, R. N.; Bhattacharjee, S.; Biju, A. T. Org. Lett. 2019, 21, 737.
} 


\section{Mechanistic Experiments}

\section{Experiments to show the role of nucleophile in the dealkylation step}

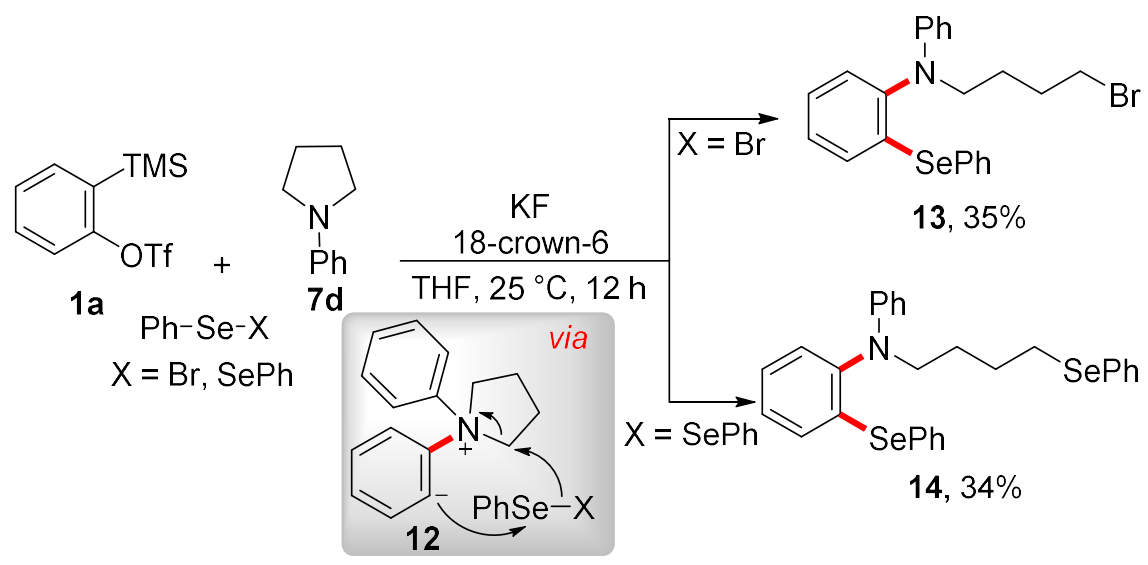

To a flame-dried screw-capped test tube equipped with a magnetic stir bar was added the 18-crown-6 (0.528 g, $2.0 \mathrm{mmol}), \mathrm{KF}(0.116 \mathrm{~g}, 2.0 \mathrm{mmol})$ inside the glove-box. Electrophilic selenium source $3 \mathbf{a}$ or $\mathbf{6 a}(0.75 \mathrm{mmol})$ was added and the mixture was dissolved in $2.0 \mathrm{~mL}$ of THF outside the glove-box under argon and kept stirring for five minutes. To this stirring mixture was added 1-phenylpyrrolidine $7 \mathbf{d}^{5}(0.074 \mathrm{~g}, 0.5 \mathrm{mmol})$ and aryne precursor $1 \mathrm{a}(0.298 \mathrm{~g}, 243 \mu \mathrm{L}, 1.0 \mathrm{mmol})$. Then the reaction mixture was allowed to react at $25{ }^{\circ} \mathrm{C}$ for $12 \mathrm{~h}$. After $12 \mathrm{~h}$, the reaction was stopped, the solvent was evaporated and the crude residue pre-adsorbed on silica gel and purified by flash column chromatography on silica gel to afforded the corresponding 2-selanyl aniline derivatives 13 and $\mathbf{1 4}$ in 35\% and $34 \%$ yield respectively.

This experiment indicates the role of nucleophile $(X=\mathrm{Br}$ or SePh) in the dealkylation step in the three-component reaction.

\section{X-ray Data of $4 f$ and 13}

Single crystal of $\mathbf{4 f}$ (recrystallized from Pet. ether at $25^{\circ} \mathrm{C}$ ) was mounted and the diffraction data was collected at $296 \mathrm{~K}$ on a Bruker SMART APEX CCD diffractometer using SMART/SAINT software. Intensity data were collected using graphite-monochromatized Mo-Ka

${ }^{5}$ Cano, R.; Ramón, D. J.; Yus, M. J. Org. Chem. 2011, 76, 654. 
radiation $(71.073 \mathrm{pm})$. The structure was solved by direct methods using the ShelXS ${ }^{6}$ and refined with ShelXS ${ }^{6}$. Empirical absorption corrections were applied with SADABS. ${ }^{7}$ All Non-hydrogen atoms were refined anisotropically and hydrogen atoms were included in geometric positions. Structure was drawn using Olex-2 and ORTEP-3. The crystallographic refinement parameters are given below:

\section{Table S2 Crystal data and structure refinement for $4 \mathrm{f}$}

\begin{tabular}{|c|c|}
\hline Identification code & 4f \\
\hline CCDC Number & 1953258 \\
\hline Empirical formula & $\mathrm{C}_{19} \mathrm{H}_{16} \mathrm{BrNSe}$ \\
\hline Formula weight & 417.20 \\
\hline Temperature/K & 296.15 \\
\hline Crystal system & monoclinic \\
\hline Space group & $\mathrm{P} 21 / \mathrm{n}$ \\
\hline $\mathrm{a} / \AA$ & $14.2571(4)$ \\
\hline $\mathrm{b} / \AA$ & $6.2778(2)$ \\
\hline $\mathrm{c} / \AA$ & $19.5208(5)$ \\
\hline$\alpha /{ }^{\circ}$ & 90.00 \\
\hline$\beta /{ }^{\circ}$ & $98.404(2)$ \\
\hline$\gamma /{ }^{\circ}$ & 90.00 \\
\hline Volume $/ \AA^{3}$ & $1728.41(9)$ \\
\hline Z & 4 \\
\hline pcalcmg $/ \mathrm{mm}^{3}$ & 1.603 \\
\hline$\mu / \mathrm{mm}^{-1}$ & 4.480 \\
\hline $\mathrm{F}(000)$ & 824.0 \\
\hline Radiation & $\operatorname{MoK} \alpha(\lambda=0.71073)$ \\
\hline Crystal size $/ \mathrm{mm}^{3}$ & $0.273 \times 0.251 \times 0.238$ \\
\hline $2 \Theta$ range for data collection & 3.32 to $55.2^{\circ}$ \\
\hline Index ranges & $-18 \leq \mathrm{h} \leq 18,-8 \leq \mathrm{k} \leq 8,-25 \leq 1 \leq 25$ \\
\hline Reflections collected & 28032 \\
\hline Independent reflections & $3991[\mathrm{R}($ int $)=0.0613]$ \\
\hline Data/restraints/parameters & $3991 / 0 / 200$ \\
\hline Goodness-of-fit on $\mathrm{F}^{2}$ & 1.012 \\
\hline Final $\mathrm{R}$ indexes & {$[\mathrm{I}>=2 \sigma(\mathrm{I})] \mathrm{R}_{1}=0.0398, \mathrm{wR}_{2}=0.0752$} \\
\hline Final $\mathrm{R}$ indexes [all data] & $\mathrm{R}_{1}=0.0847, \mathrm{wR}_{2}=0.0868$ \\
\hline Largest diff. peak/hole / e $\AA^{-3}$ & $0.59 /-0.78$ \\
\hline
\end{tabular}

${ }^{6}$ SHELXS, G.M. Sheldrick, Acta Cryst. 2008, A64, 112.

${ }^{7}$ Sheldrick, G. M. SADABS, University of Göttingen, Göttingen, Germany, 1999. 


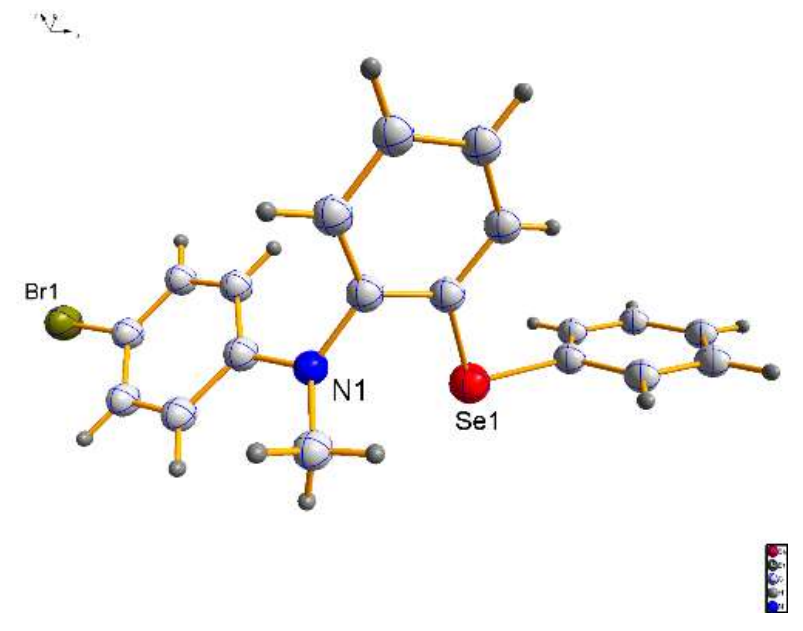

Figure S1. Crystal Structure of $\mathbf{4 f}$ (Thermal ellipsoids are shown with 50\% probability)

\section{X-ray data of 13}

Single crystal of $\mathbf{1 3}$ (recrystallized from DCM/Pet Ether at $25^{\circ} \mathrm{C}$ ) was mounted and the diffraction data was collected at $298 \mathrm{~K}$ on a Bruker SMART APEX CCD diffractometer using SMART/SAINT software. Intensity data were collected using graphite-monochromatized Mo-Ka radiation $(71.073 \mathrm{pm})$. The structure was solved by direct methods using the ShelXS ${ }^{6}$ and refined with ShelXS. Empirical absorption corrections were applied with SADABS. ${ }^{7}$ All Non-hydrogen atoms were refined anisotropically and hydrogen atoms were included in geometric positions. Structure was drawn using Olex-2 and ORTEP-3. The crystallographic refinement parameters are given below:

\section{Table S3 Crystal data and structure refinement for}

$\begin{array}{ll}\text { Identification code } & \mathbf{1 3} \\ \text { CCDC no } & 1961216 \\ \text { Empirical formula } & \mathrm{C}_{22} \mathrm{H}_{22} \mathrm{BrNSe} \\ \text { Formula weight } & 459.289 \\ \text { Temperature/K } & 298 \\ \text { Crystal system } & \text { triclinic } \\ \text { Space group } & \mathrm{P}-1 \\ \mathrm{a} / \AA & 8.6022(8) \\ \mathrm{b} / \AA & 10.7583(10) \\ \mathrm{c} / \AA & 11.7185(11) \\ \alpha /{ }^{\circ} & 73.280(4) \\ \beta /{ }^{\circ} & 74.058(4) \\ \gamma^{\circ} & 82.430(4) \\ \text { Volume } / \AA^{3} & 997.06(16) \\ \mathrm{Z} & 2\end{array}$


$\rho_{\text {calc } \mathrm{mg} / \mathrm{mm}^{3}}$

$\mu / \mathrm{mm}^{-1}$

$\mathrm{F}(000)$

Crystal size $/ \mathrm{mm}^{3}$

$2 \Theta$ range for data collection

Index ranges

Reflections collected

Independent reflections

Data/restraints/parameters

Goodness-of-fit on

Final $\mathrm{R}$ indexes

Final $\mathrm{R}$ indexes [all data]

Largest diff. peak/hole / e $\AA^{-3}$
1.530

3.891

460.0

$0.6 \times 0.5 \times 0.4$

3.748 to 49.998

$-10 \leq \mathrm{h} \leq 10,-12 \leq \mathrm{k} \leq 12,-13 \leq 1 \leq 13$

24565

$3510[$ Rint $=0.0383$, Rsigma $=0.0236]$

$3510 / 1 / 226$

1.033

$\mathrm{R} 1=0.0898, \mathrm{wR} 2=0.2104$

$\mathrm{R} 1=0.1097, \mathrm{wR} 2=0.2210$

$1.88 /-2.14$

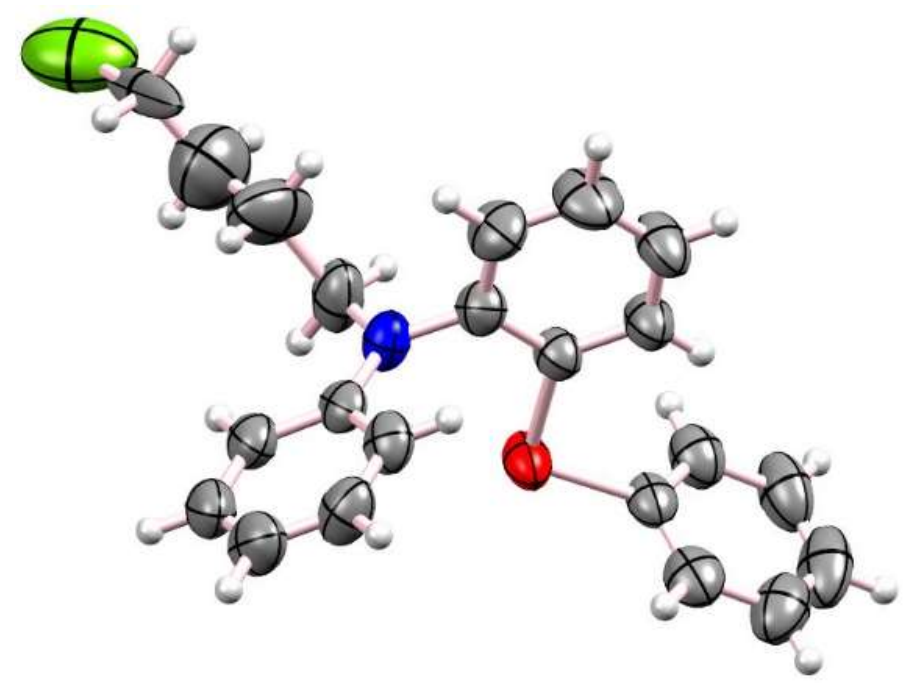

Figure S2. Crystal Structure of $\mathbf{1 3}$ 


\section{Synthesis and Characterization of 2-Selanyl Aniline Derivatives}

\section{$N$-Methyl- $N$-phenyl-2-(phenylselanyl)aniline (4a)}<smiles>CN(c1ccccc1)c1ccccc1[Se]c1ccccc1</smiles>

Following the general procedure, treatment of 2-(trimethylsilyl)phenyl trifluoromethanesulfonate $1 \mathrm{a}(0.298 \mathrm{~g}, 243 \mu \mathrm{L}, 1.0 \mathrm{mmol})$ and $\mathrm{N}, \mathrm{N}$ dimethylaniline 2a $(0.061 \mathrm{~g}, 63 \mu \mathrm{L}, 0.5 \mathrm{mmol})$ with Phenylselenyl bromide 3a $(0.177 \mathrm{~g}, 0.75 \mathrm{mmol})$ in the presence of $\mathrm{KF}(0.116 \mathrm{~g}, 2.0 \mathrm{mmol})$ and 18 crown-6 $(0.528 \mathrm{~g}, 2.0 \mathrm{mmol})$ in THF $(2.0 \mathrm{~mL})$ at $25{ }^{\circ} \mathrm{C}$ for $12 \mathrm{~h}$ followed by flash column chromatography (Pet.ether/DCM = 95/05) of the crude reaction mixture using silica gel afforded $N$-methyl- $N$-phenyl-2-(phenylselanyl)aniline $4 \mathbf{a}$ as a white solid $(0.121 \mathrm{~g}, 72 \%$ yield $)$.

$\boldsymbol{R}_{\mathbf{f}}\left(\right.$ Pet. ether /DCM = 95/05): 0.44; ${ }^{1} \mathbf{H}$ NMR (400 MHz, $\left.\mathbf{C D C l}_{3}\right) \delta$ 7.67-7.65 (m, 2H), 7.42-7.35 $(\mathrm{m}, 3 \mathrm{H}), 7.26-7.21(\mathrm{~m}, 3 \mathrm{H}), 7.16\left(\mathrm{dd}, J_{1}=7.8 \mathrm{~Hz}, J_{2}=1.4 \mathrm{~Hz}, 1 \mathrm{H}\right), 7.11-7.07(\mathrm{~m}, 1 \mathrm{H}), 7.04$ $\left(\mathrm{dd}, J_{l}=7.9 \mathrm{~Hz}, J_{2}=1.4 \mathrm{~Hz}, 1 \mathrm{H}\right), 6.81(\mathrm{t}, J=7.3 \mathrm{~Hz}, 1 \mathrm{H}), 6.68(\mathrm{~d}, J=8.0 \mathrm{~Hz}, 2 \mathrm{H}), 3.27(\mathrm{~s}, 3 \mathrm{H})$. ${ }^{13}$ C NMR (100 MHz, CDCl $) \delta$ 149.2, 146.9, 136.6, 136.3, 130.3, 129.7, 129.1, 128.6, 128.5, 128.4, 127.7, 127.6, 118.1, 114.0, 39.2. HRMS (ESI) calculated $[\mathrm{M}+\mathrm{H}]{ }^{+}$for $\mathrm{C}_{19} \mathrm{H}_{18} \mathrm{NSe}$ : 340.0599, found: 340.0606. FTIR (cm $\left.{ }^{-1}\right)$ 3057, 2922, 1601, 1497, 1336, 1022.

\section{$\mathrm{N}$-(4-Methoxyphenyl)- $\mathrm{N}$-methyl-2-(phenylselanyl)aniline (4b)}

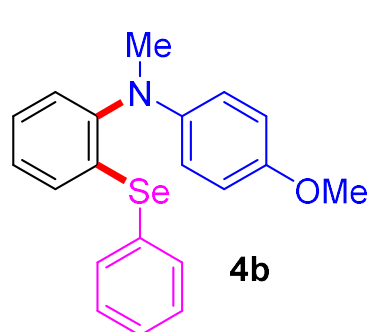

Following the general procedure, treatment of 2-(trimethylsilyl)phenyl trifluoromethanesulfonate $1 \mathrm{a}(0.298 \mathrm{~g}, 243 \mu \mathrm{L}, 1.0 \mathrm{mmol})$ and 4methoxy- $N, N$-dimethylaniline $\quad \mathbf{2 b} \quad(0.076 \quad \mathrm{~g}, \quad 0.5 \quad \mathrm{mmol})$ with Phenylselenyl bromide 3a $(0.177 \mathrm{~g}, 0.75 \mathrm{mmol})$ in the presence of $\mathrm{KF}$ $(0.116 \mathrm{~g}, 2.0 \mathrm{mmol})$ and 18-crown-6 $(0.528 \mathrm{~g}, 2.0 \mathrm{mmol})$ in THF (2.0 $\mathrm{mL}$ ) at $25{ }^{\circ} \mathrm{C}$ for $12 \mathrm{~h}$ followed by flash column chromatography (Pet.ether/DCM $=85 / 15$ ) of the crude reaction mixture using silica gel afforded $N$-(4-methoxyphenyl)- $N$-methyl-2(phenylselanyl)aniline $\mathbf{4 b}$ as a white solid ( $0.135 \mathrm{~g}, 73 \%$ yield).

$\boldsymbol{R}_{\mathbf{f}}($ Pet. ether $/ \mathrm{DCM}=90 / 10)$ : 0.24; ${ }^{1} \mathbf{H}$ NMR (400 $\left.\mathbf{M H z}, \mathbf{C D C l}_{3}\right) \delta$ 7.67-7.64(m, 2H), 7.427.34(m, 3H), 7.21-7.17 (m, 1H), 7.11(dd, $\left.J_{l}=7.8 \mathrm{~Hz}, J_{2}=1.4 \mathrm{~Hz}, 1 \mathrm{H}\right), 7.06-7.02(\mathrm{~m}, 1 \mathrm{H}), 6.98$ $\left(\mathrm{dd}, J_{1}=7.9 \mathrm{~Hz}, J_{2}=1.5 \mathrm{~Hz}, 1 \mathrm{H}\right), 6.84-6.80(\mathrm{~m}, 2 \mathrm{H}), 6.69-6.65(\mathrm{~m}, 2 \mathrm{H}), 3.78(\mathrm{~s}, 3 \mathrm{H}), 3.21(\mathrm{~s}$, 3H). ${ }^{13} \mathbf{C}$ NMR (100 MHz, $\left.\mathbf{C D C l}_{3}\right) \delta 152.8,147.8,143.8,136.7,135.9,130.0,129.6,128.6$, 128.5, 127.6, 127.5, 127.0, 116.2, 114.6, 55.8, 40.0. HRMS (ESI) calculated $[\mathrm{M}+\mathrm{H}]{ }^{+}$for 
$\mathrm{C}_{20} \mathrm{H}_{20}$ NOSe: 370.0705 , found: 370.0690. FTIR (cm $\left.{ }^{-1}\right)$ 3053, 2950, 1576, 1508, 1469, 1241, 1037.

\section{$N$-Methyl-2-(phenylselanyl)- $N$-(p-tolyl)aniline (4c)}<smiles>Cc1ccc(N(C)c2ccccc2[Se]c2ccccc2)cc1</smiles>

Following the general procedure, treatment of 2-(trimethylsilyl)phenyl trifluoromethanesulfonate $1 \mathrm{a}(0.149 \mathrm{~g}, 121 \mu \mathrm{L}, 0.5 \mathrm{mmol})$ and $N, N, 4-$ trimethylaniline $2 \mathrm{c}(0.034 \mathrm{~g}, 0.25 \mathrm{mmol})$ with Phenylselenyl bromide 3a $(0.088 \mathrm{~g}, 0.375 \mathrm{mmol})$ in the presence of $\mathrm{KF}(0.058 \mathrm{~g}, 1.0 \mathrm{mmol})$ and 18 crown-6 (0.264 g, $1.0 \mathrm{mmol})$ in THF $(1.0 \mathrm{~mL})$ at $25^{\circ} \mathrm{C}$ for $12 \mathrm{~h}$ followed by flash column chromatography (Pet.ether/DCM $=95 / 05$ ) of the crude reaction mixture using silica gel afforded $N$-methyl-2-(phenylselanyl)- $N$-(p-tolyl)aniline $4 \mathbf{c}$ as a pale yellow oil (0.060 g, 68\% yield).

$\boldsymbol{R}_{\mathbf{f}}\left(\right.$ Pet. ether $/ \mathrm{DCM}=$ 95/05): 0.49; ${ }^{1} \mathbf{H}$ NMR (400 $\left.\mathbf{M H z}, \mathbf{C D C l}_{3}\right) \delta$ 7.68-7.65 (m, 2H), 7.43 $7.35(\mathrm{~m}, 3 \mathrm{H}), 7.23-7.19(\mathrm{~m}, 1 \mathrm{H}), 7.14\left(\mathrm{dd}, J_{1}=7.9 \mathrm{~Hz}, J_{2}=1.3 \mathrm{~Hz}, 1 \mathrm{H}\right), 7.09-7.04(\mathrm{~m}, 3 \mathrm{H}), 7.01$ $\left(\mathrm{dd}, J_{l}=7.9 \mathrm{~Hz}, J_{2}=1.3 \mathrm{~Hz}, 1 \mathrm{H}\right), 6.61(\mathrm{~d}, J=8.5 \mathrm{~Hz}, 2 \mathrm{H}), 3.25(\mathrm{~s}, 3 \mathrm{H}), 2.30(\mathrm{~s}, 3 \mathrm{H}) .{ }^{13} \mathbf{C} \mathbf{~ N M R}$ (100 MHz, CDCl 3$) \delta 147.3,147.1,136.6,136.3,130.1,129.64,129.62,128.6,128.5,128.2$, 127.6, 127.4, 127.3, 114.4, 39.5, 20.5. HRMS (ESI) calculated $[\mathrm{M}+\mathrm{H}]{ }^{+}$for $\mathrm{C}_{20} \mathrm{H}_{20} \mathrm{NSe}$ : 354.0755, found: 354.0765. FTIR (cm-1) 3449, 3058, 1614, 1574, 1512, 1470, 1021.

\section{$N$-(4-Ethylphenyl)- $N$-methyl-2-(phenylselanyl)aniline (4d)}<smiles>CCc1ccc(N(C)c2ccccc2[Se]c2ccccc2)cc1</smiles>

Following the general procedure, treatment of 2 (trimethylsilyl)phenyl trifluoromethanesulfonate 1a $(0.298 \mathrm{~g}, 243 \mu \mathrm{L}, 1.0 \mathrm{mmol})$ and 4-ethyl$N, N$-dimethylaniline 2d $(0.075 \mathrm{~g}, 0.5 \mathrm{mmol})$ with Phenylselenyl bromide

3a $(0.177 \mathrm{~g}, 0.75 \mathrm{mmol})$ in the presence of $\mathrm{KF}(0.116 \mathrm{~g}, 2.0 \mathrm{mmol})$ and 18-crown-6 $(0.528 \mathrm{~g}, 2.0 \mathrm{mmol})$ in THF $(2.0 \mathrm{~mL})$ at $25{ }^{\circ} \mathrm{C}$ for $12 \mathrm{~h}$ followed by flash column chromatography (Pet.ether/DCM $=95 / 05$ ) of the crude reaction mixture using silica gel afforded $N$-(4-ethylphenyl)- $N$-methyl-2-(phenylselanyl)aniline $\mathbf{4 d}$ as a pale yellow oil ( $0.135 \mathrm{~g}, 74 \%$ yield $)$.

$\boldsymbol{R}_{\mathbf{f}}($ Pet. ether $/ \mathrm{DCM}=95 / 05)$ : 0.50; ${ }^{1} \mathbf{H}$ NMR (400 $\left.\mathbf{M H z}, \mathbf{C D C l}_{3}\right) \delta$ 7.72-7.69 (m, 2H), 7.45$7.38(\mathrm{~m}, 3 \mathrm{H}), 7.26-7.22(\mathrm{~m}, 1 \mathrm{H}), 7.18\left(\mathrm{dd}, J_{1}=7.8 \mathrm{~Hz}, J_{2}=1.6 \mathrm{~Hz}, 1 \mathrm{H}\right), 7.13-7.08(\mathrm{~m}, 3 \mathrm{H}), 7.04$ $\left(\mathrm{dd}, J_{1}=7.9 \mathrm{~Hz}, J_{2}=1.4 \mathrm{~Hz}, 1 \mathrm{H}\right), 6.69-6.66(\mathrm{~m}, 2 \mathrm{H}), 3.28(\mathrm{~s}, 3 \mathrm{H}), 2.64(\mathrm{q}, J=7.7 \mathrm{~Hz}, 2 \mathrm{H}), 1.27$ 


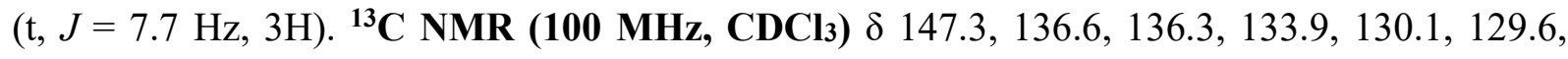
129.1, 128.8, 128.6, 128.4, 128.3, 127.5, 127.3, 114.3, 39.4, 28.0, 15.9. HRMS (ESI) calculated $[\mathrm{M}+\mathrm{H}]^{+}$for $\mathrm{C}_{21} \mathrm{H}_{22} \mathrm{NSe}: 368.0912$, found: 368.0915. FTIR $\left(\mathbf{c m}^{-1}\right)$ 3450, 2961, 2866, 1613, $1512,1333$.

\section{$N$-(4-Iodophenyl)- $N$-methyl-2-(phenylselanyl)aniline (4e)}<smiles>CN(c1ccc(I)cc1)c1ccccc1[Se]c1ccccc1</smiles>

Following the general procedure, treatment of 2-(trimethylsilyl)phenyl trifluoromethanesulfonate 1a $(0.298 \mathrm{~g}, 243 \mu \mathrm{L}, 1.0 \mathrm{mmol})$ and 4-iodo- $N, N$ dimethylaniline 2e $(0.124 \mathrm{~g}, 0.5 \mathrm{mmol})$ with Phenylselenyl bromide 3a $(0.177 \mathrm{~g}, 0.75 \mathrm{mmol})$ in the presence of $\operatorname{KF}(0.116 \mathrm{~g}, 2.0 \mathrm{mmol})$ and 18 crown-6 $(0.528 \mathrm{~g}, 2.0 \mathrm{mmol})$ in THF $(2.0 \mathrm{~mL})$ at $25^{\circ} \mathrm{C}$ for $12 \mathrm{~h}$ followed by flash column chromatography (Pet.ether/DCM = 97/03) of the crude reaction mixture using silica gel afforded $N$-(4-iodophenyl)- $N$-methyl-2-(phenylselanyl)aniline $4 \mathbf{e}$ as a pale yellow oil (0.147 g,63\% yield).

$\boldsymbol{R}_{\mathbf{f}}\left(\right.$ Pet. ether $/ \mathrm{DCM}=$ 95/05): 0.60; ${ }^{1} \mathbf{H}$ NMR (400 MHz, $\left.\mathbf{C D C l}_{3}\right) \delta$ 7.65-7.62 (m, 2H), 7.47 (d, $J$ $=8.8 \mathrm{~Hz}, 2 \mathrm{H}), 7.41-7.36(\mathrm{~m}, 3 \mathrm{H}), 7.25-7.21(\mathrm{~m}, 1 \mathrm{H}), 7.14-7.09(\mathrm{~m}, 2 \mathrm{H}), 7.04\left(\mathrm{dd}, J_{1}=7.8 \mathrm{~Hz}, J_{2}\right.$ $=1.2 \mathrm{~Hz}, 1 \mathrm{H}), 6.42(\mathrm{~d}, J=8.8 \mathrm{~Hz}, 2 \mathrm{H}), 3.23(\mathrm{~s}, 3 \mathrm{H}) .{ }^{13} \mathbf{C}$ NMR (125 MHz, CDCl$) \delta ~ 148.6$, 146.0, 137.6, 136.4, 136.1, 130.6, 129.7, 128.7, 128.5, 128.1, 127.9, 127.8, 116.0, 79.4, 39.1. HRMS (ESI) calculated $[\mathrm{M}+\mathrm{H}]^{+}$for $\mathrm{C}_{19} \mathrm{H}_{17} \mathrm{INSe}: 465.9565$, found: 465.9568. FTIR (cm ${ }^{-}$ 1) $3057,2871,1576,1489,1340,1115$.

\section{$\mathrm{N}$-(4-Bromophenyl)- $\mathrm{N}$-methyl-2-(phenylselanyl)aniline (4f)}<smiles>CN(c1ccc(Br)cc1)c1ccccc1[Se]c1ccccc1</smiles>

Following the general procedure, treatment of 2-(trimethylsilyl)phenyl trifluoromethanesulfonate $1 \mathrm{a}(0.149 \mathrm{~g}, 121 \mu \mathrm{L}, 0.5 \mathrm{mmol})$ and 4-bromo$N, N$-dimethylaniline $2 \mathbf{f}(0.050 \mathrm{~g}, 0.25 \mathrm{mmol})$ with Phenylselenyl bromide 3a $(0.088 \mathrm{~g}, 0.375 \mathrm{mmol})$ in the presence of $\mathrm{KF}(0.058 \mathrm{~g}, 1.0 \mathrm{mmol})$ and 18-crown-6 $(0.264 \mathrm{~g}, 1.0 \mathrm{mmol})$ in THF $(1.0 \mathrm{~mL})$ at $25{ }^{\circ} \mathrm{C}$ for $12 \mathrm{~h}$ followed by flash column chromatography (Pet.ether/DCM $=97 / 03$ ) of the crude reaction mixture using silica gel afforded $N$-(4-bromophenyl)- $N$-methyl-2-(phenylselanyl)aniline $4 \mathbf{f}$ as a white solid (0.064 g,62\% yield). 
$\boldsymbol{R}_{\mathbf{f}}($ Pet. ether $/ \mathrm{DCM}=95 / 05)$ : 0.60; ${ }^{1} \mathbf{H}$ NMR (400 MHz, $\left.\mathbf{C D C l}_{3}\right) \delta$ 7.67-7.65 (m, 2H), 7.437.37(m, 3H), 7.34-7.23 (m, 3H), 7.16-7.11(m, 2H), 7.07-7.04 (m, 1H), 6.56-6.52 (m, 2H), 3.25 (s, 3H). ${ }^{13} \mathbf{C}$ NMR (100 MHz, $\left.\mathbf{C D C l}_{3}\right) \delta 148.1,146.1,136.5,136.2,131.7,130.4,129.7,128.8$, 128.5, 128.0, 127.9, 127.8, 115.4, 110.1, 39.2. HRMS (ESI) calculated $[\mathrm{M}+\mathrm{H}]{ }^{+}$for $\mathrm{C}_{19} \mathrm{H}_{17} \mathrm{BrNSe}: 417.9704$, found:417.9704. FTIR ( cm$\left.^{-1}\right)$ 3415, 2922, 1573, 1489, 1337, 1115.

\section{$N$-(4-Chlorophenyl)- $N$-methyl-2-(phenylselanyl)aniline (4g)}<smiles>CN(c1ccc(Cl)cc1)c1ccccc1[Se]c1ccccc1</smiles>

Following the general procedure, treatment of 2-(trimethylsilyl)phenyl trifluoromethanesulfonate $1 \mathrm{a}(0.298 \mathrm{~g}, 243 \mu \mathrm{L}, 1.0 \mathrm{mmol})$ and 4-chloro$N, N$-dimethylaniline $2 \mathrm{~g}(0.078 \mathrm{~g}, 0.5 \mathrm{mmol})$ with Phenylselenyl bromide 3a $(0.177 \mathrm{~g}, 0.75 \mathrm{mmol})$ in the presence of $\mathrm{KF}(0.116 \mathrm{~g}, 2.0 \mathrm{mmol})$ and 18-crown-6 $(0.528 \mathrm{~g}, 2.0 \mathrm{mmol})$ in THF $(2.0 \mathrm{~mL})$ at $25{ }^{\circ} \mathrm{C}$ for $12 \mathrm{~h}$ followed by flash column chromatography (Pet.ether/DCM $=95 / 05)$ of the crude reaction mixture using silica gel afforded $N$-(4-chlorophenyl)- $N$-methyl-2(phenylselanyl)aniline $4 \mathrm{~g}$ as a white solid (0.119 g, 64\% yield).

$\boldsymbol{R}_{\mathbf{f}}$ (Pet. ether /DCM = 95/05): 0.60; ${ }^{1} \mathbf{H}$ NMR (400 MHz, $\left.\mathbf{C D C l}_{3}\right) \delta$ 7.68-7.65 (m, 2H), 7.46-7.36 (m, 3H), 7.28-7.24 (m, 1H), 7.21-7.10 (m, 4H), 7.06 (dd, $\left.J_{l}=7.9 \mathrm{~Hz}, J_{2}=1.5 \mathrm{~Hz}, 1 \mathrm{H}\right), 6.61-6.58$

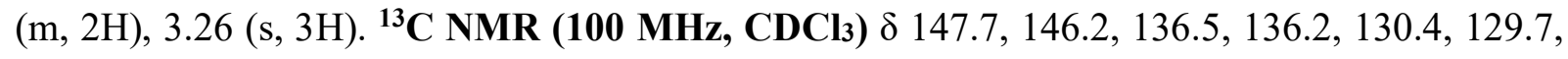
$128.9,128.7,128.5,128.0,127.9,127.8,122.9,115.0$, 39.3. HRMS (ESI) calculated $[\mathrm{M}+\mathrm{H}]^{+}$ for $\mathrm{C}_{19} \mathrm{H}_{17} \mathrm{ClNSe}$ 374.0209, found: 374.0211. FTIR (cm $\left.{ }^{-1}\right)$ 3056, 2873, 1597, 1492, 1338, 111 .

\section{$N$-(4-Fluorophenyl)- $N$-methyl-2-(phenylselanyl)aniline (4h)}<smiles>CN(c1ccc(F)cc1)c1ccccc1[Se]c1ccccc1</smiles>

Following the general procedure, treatment of 2-(trimethylsilyl)phenyl trifluoromethanesulfonate $1 \mathrm{a}(0.298 \mathrm{~g}, 243 \mu \mathrm{L}, 1.0 \mathrm{mmol})$ and 4-fluoro$N, N$-dimethylaniline $\mathbf{2 h}(0.070 \mathrm{~g}, 0.5 \mathrm{mmol})$ with Phenylselenyl bromide 3a $(0.177 \mathrm{~g}, 0.75 \mathrm{mmol})$ in the presence of $\mathrm{KF}(0.116 \mathrm{~g}, 2.0 \mathrm{mmol})$ and 18 crown-6 (0.528 g, $2.0 \mathrm{mmol})$ in THF $(2.0 \mathrm{~mL})$ at $25^{\circ} \mathrm{C}$ for $12 \mathrm{~h}$ followed by flash column chromatography (Pet.ether/DCM $=95 / 05$ ) of the crude reaction mixture using silica gel afforded $N$-(4-fluorophenyl)- $N$-methyl-2-(phenylselanyl)aniline $\mathbf{4 h}$ as a light yellow solid (0.126 g,70\% yield). 
$\boldsymbol{R}_{\mathbf{f}}$ (Pet. ether /DCM = 95/05): 0.60; ${ }^{1} \mathbf{H}$ NMR (400 MHz, $\left.\mathbf{C D C l}_{3}\right) \delta$ 7.66-7.63 (m, 2H), 7.42-7.34 (m, 3H), 7.23-7.19 (m, 1H), 7.13-7.05 (m, 2H), 7.01 (dd, $\left.J_{1}=7.9 \mathrm{~Hz}, J_{2}=1.5 \mathrm{~Hz}, 1 \mathrm{H}\right), 6.95-6.89$ (m, 2H), 6.63-6.57 (m, 2H), 3.23 (s, 3H). ${ }^{13} \mathbf{C}$ NMR (100 MHz, CDCl 3 ) $\delta 156.9(\mathrm{~d}, J=236.4$ $\mathrm{Hz}), 147.2,145.8$ (d, $J=1.4 \mathrm{~Hz}), 136.6,136.1,130.4,129.7,128.7,128.3,128.2,127.6$ (d, $J=$ $20.5 \mathrm{~Hz}), 116.0,115.3(\mathrm{~d}, J=3.5 \mathrm{~Hz}), 115.3,39.8$. HRMS (ESI) calculated $[\mathrm{M}+\mathrm{H}]{ }^{+}$for $\mathrm{C}_{19} \mathrm{H}_{17} \mathrm{FNSe}: 358.0505$, found: 358.0515. FTIR (cm $\left.{ }^{-1}\right)$ 3054, 2870, 1507, 1470, 1335, 1225, 1023.

\section{$N$-Methyl-2-(phenylselanyl)- $N$-(4-(trifluoromethyl)phenyl)aniline (4i)}

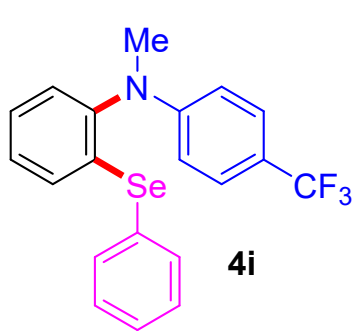

Following the general procedure, treatment of 2-(trimethylsilyl)phenyl trifluoromethanesulfonate $1 \mathrm{a}(0.149 \mathrm{~g}, 121 \mu \mathrm{L}, 0.5 \mathrm{mmol})$ and $N, N$ dimethyl-4-(trifluoromethyl)aniline $2 \mathbf{2 i}(0.047 \mathrm{~g}, 0.25 \mathrm{mmol})$ with Phenylselenyl bromide 3a $(0.088 \mathrm{~g}, 0.375 \mathrm{mmol})$ in the presence of $\mathrm{KF}$

$(0.058 \mathrm{~g}, 1.0 \mathrm{mmol})$ and 18 -crown-6 $(0.264 \mathrm{~g}, 1.0 \mathrm{mmol})$ in THF $(1.0$ $\mathrm{mL}$ ) at $65^{\circ} \mathrm{C}$ for $12 \mathrm{~h}$ followed by flash column chromatography (Pet.ether/DCM $=95 / 05$ ) of the crude reaction mixture using silica gel afforded $N$-methyl-2-(phenylselanyl)- $N$-(4(trifluoromethyl)phenyl)aniline $4 \mathbf{i}$ as a pale yellow oil (0.028 g, 28\% yield).

$\boldsymbol{R}_{\mathbf{f}}($ Pet. ether $/ \mathrm{DCM}=95 / 05)$ : 0.53; ${ }^{1} \mathbf{H}$ NMR (400 MHz, $\left.\mathbf{C D C l}_{3}\right) \delta$ 7.60-7.58 (m, 2H), $7.42(\mathrm{~d}, J$ $=8.7 \mathrm{~Hz}, 2 \mathrm{H}), 7.39-7.32(\mathrm{~m}, 3 \mathrm{H}), 7.25-7.23(\mathrm{~m}, 1 \mathrm{H}), 7.15-7.11(\mathrm{~m}, 2 \mathrm{H}), 7.08-7.06(\mathrm{~m}, 1 \mathrm{H})$, $6.60(\mathrm{~d}, J=8.6 \mathrm{~Hz}, 2 \mathrm{H}), 3.27$ (s, 3H). ${ }^{13} \mathbf{C}$ NMR (100 MHz, CDCl 3$) \delta 151.2,145.5,136.4$, 136.1, 131.0, 129.8, 128.9, 128.8, 128.3, 128.1, 127.9, 136.4 (q, $J=3.5 \mathrm{~Hz}), 125.2$ (q, $J=270.0$ $\mathrm{Hz}), 119.3(\mathrm{q}, J=33.0 \mathrm{~Hz}), 112.7,39.0$. HRMS (ESI) calculated $[\mathrm{M}+\mathrm{H}]^{+}$for $\mathrm{C}_{20} \mathrm{H}_{17} \mathrm{~F}_{3} \mathrm{NSe}$ : 408.0473. found: 408.0479. FTIR (cm $\left.{ }^{-1}\right)$ 3058, 2924, 2363, 1616, 1472, 1326, 1111.

\section{$N$-Methyl-2-(phenylselanyl)- $N$-(m-tolyl)aniline $(4 \mathbf{j})$}<smiles>Cc1cccc(N(C)c2ccccc2[Se]c2ccccc2)c1</smiles>

Following the general procedure, treatment of 2-(trimethylsilyl)phenyl trifluoromethanesulfonate $1 \mathrm{a}(0.298 \mathrm{~g}, 243 \mu \mathrm{L}, 1.0 \mathrm{mmol})$ and $N, N, 3-$ trimethylaniline $2 \mathbf{j}$ ( $0.068 \mathrm{~g}, 0.5 \mathrm{mmol})$ with Phenylselenyl bromide 3a $(0.177 \mathrm{~g}, 0.75 \mathrm{mmol})$ in the presence of KF $(0.116 \mathrm{~g}, 2.0 \mathrm{mmol})$ and 18 crown-6 $(0.528 \mathrm{~g}, 2.0 \mathrm{mmol})$ in THF $(2.0 \mathrm{~mL})$ at $25^{\circ} \mathrm{C}$ for $12 \mathrm{~h}$ followed by flash column chromatography (Pet.ether/DCM $=95 / 05)$ of the crude reaction mixture using 
silica gel afforded $N$-methyl-2-(phenylselanyl)- $N$-( $m$-tolyl)aniline $\mathbf{4 j}$ as a white solid $(0.101 \mathrm{~g}$, $57 \%$ yield).

$\boldsymbol{R}_{\mathbf{f}}$ (Pet. ether /DCM = 95/05): 0.50; ${ }^{1} \mathbf{H}$ NMR (400 MHz, $\left.\mathbf{C D C l}_{3}\right) \delta$ 7.72-7.70 (m, 2H), 7.47-7.39 $(\mathrm{m}, 3 \mathrm{H}), 7.28-7.24(\mathrm{~m}, 1 \mathrm{H}), 7.21-7.11(\mathrm{~m}, 3 \mathrm{H}), 7.07\left(\mathrm{dd}, J_{1}=7.9 \mathrm{~Hz}, J_{2}=1.3 \mathrm{~Hz}, 1 \mathrm{H}\right), 6.69(\mathrm{~d}, J$ $=7.9 \mathrm{~Hz}, 1 \mathrm{H}), 6.56(\mathrm{~s}, 1 \mathrm{H}), 6.54-6.52(\mathrm{~m}, 1 \mathrm{H}), 3.30(\mathrm{~s}, 3 \mathrm{H}), 2.36(\mathrm{~s}, 3 \mathrm{H}) .{ }^{13} \mathbf{C}$ NMR (125 MHz, $\left.\mathbf{C D C l}_{3}\right) \delta 149.2,147.0,138.7,136.5,136.3,130.3,129.6,128.9,128.6,128.5,128.4,127.6$, 127.5, 119.1, 114.7, 111.4, 39.3, 22.0. HRMS (ESI) calculated $[\mathrm{M}+\mathrm{H}]{ }^{+}$for $\mathrm{C}_{20} \mathrm{H}_{20} \mathrm{NSe}$ : 354.0755, found: 354.0760. FTIR (cm-1) 3052, 2917, 1604, 1573, 1463, 1339, 1027.

\section{$N$-(3-Chlorophenyl)- $N$-methyl-2-(phenylselanyl)aniline (4k)}

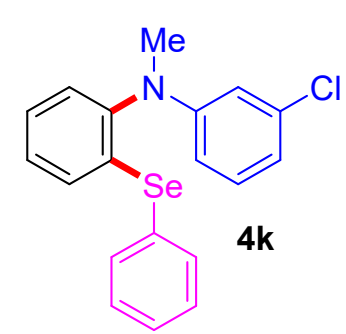

Following the general procedure, treatment of 2-(trimethylsilyl)phenyl trifluoromethanesulfonate $1 \mathrm{a}(0.298 \mathrm{~g}, 243 \mu \mathrm{L}, 1.0 \mathrm{mmol})$ and 3-chloro$N, N$-dimethylaniline $2 \mathbf{k}(0.078 \mathrm{~g}, 0.5 \mathrm{mmol})$ with Phenylselenyl bromide 3a $(0.177 \mathrm{~g}, 0.75 \mathrm{mmol})$ in the presence of $\mathrm{KF}(0.116 \mathrm{~g}, 2.0 \mathrm{mmol})$ and 18-crown-6 (0.528 g, $2.0 \mathrm{mmol})$ in THF $(2.0 \mathrm{~mL})$ at $25{ }^{\circ} \mathrm{C}$ for $12 \mathrm{~h}$ followed by flash column chromatography (Pet.ether/DCM $=95 / 05)$ of the crude reaction mixture using silica gel afforded $N$-(3-chlorophenyl)- $N$-methyl-2-(phenylselanyl)aniline $4 \mathbf{k}$ as a light yellow oil (0.092 g, 49\% yield).

$\boldsymbol{R}_{\mathbf{f}}\left(\right.$ Pet. ether /DCM = 95/05): 0.50; ${ }^{1} \mathbf{H}$ NMR (400 MHz, $\left.\mathbf{C D C l}_{3}\right) \delta$ 7.63-7.61 (m, 2H), 7.39-7.32 (m, 3H), 7.25-7.21 (m, 1H), 7.13-7.08 (m, 3H), 7.04-7.02 (m, 1H), 6.65-6.73 (m, 1H), $6.61(\mathrm{t}, J$ $=2.1 \mathrm{~Hz}, 1 \mathrm{H}), 6.47\left(\mathrm{dd}, J_{1}=8.4 \mathrm{~Hz}, J_{2}=2.3 \mathrm{~Hz}, 1 \mathrm{H}\right), 3.23(\mathrm{~s}, 3 \mathrm{H}) .{ }^{13} \mathbf{C}$ NMR (125 MHz, $\left.\mathbf{C D C l}_{3}\right) \delta 150.2,146.0,136.4,136.1,135.0,134.1,133.1,130.8,130.0,129.7,128.7,128.0$, 127.9, 117.8, 113.5, 112.0, 39.2. HRMS (ESI) calculated $[\mathrm{M}+\mathrm{H}]{ }^{+}$for $\mathrm{C}_{19} \mathrm{H}_{17} \mathrm{ClNSe}: 374.0209$, found: 374.0209. FTIR (cm $\left.{ }^{-1}\right)$ 3057, 2922, 2363, 1596, 1481, 1341.

\section{$N$-(3-Bromophenyl)- $N$-methyl-2-(phenylselanyl)aniline (4I)}<smiles>CN(c1cccc(Br)c1)c1ccccc1[Se]c1ccccc1</smiles>

Following the general procedure, treatment of 2-(trimethylsilyl)phenyl trifluoromethanesulfonate 1a $(0.298 \mathrm{~g}, 243 \mu \mathrm{L}, 1.0 \mathrm{mmol})$ and3-bromo$N, N$-dimethylaniline 21 (0.100 g, $0.5 \mathrm{mmol})$ with Phenylselenyl bromide 3a $(0.177 \mathrm{~g}, 0.75 \mathrm{mmol})$ in the presence of $\mathrm{KF}(0.116 \mathrm{~g}, 2.0 \mathrm{mmol})$ and 18-crown-6 $(0.528 \mathrm{~g}, 2.0 \mathrm{mmol})$ in THF $(2.0 \mathrm{~mL})$ at $25{ }^{\circ} \mathrm{C}$ for $12 \mathrm{~h}$ 
followed by flash column chromatography (Pet.ether/DCM $=95 / 05$ ) of the crude reaction mixture using silica gel afforded $N$-(3-bromophenyl)- $N$-methyl-2-(phenylselanyl)aniline $\mathbf{4 l}$ as a sticky liquid (0.104 g, 50\% yield).

$\boldsymbol{R}_{\mathbf{f}}$ (Pet. ether /DCM = 95/05): 0.53; ${ }^{1} \mathbf{H}$ NMR (400 MHz, $\left.\mathbf{C D C l}_{3}\right) \delta$ 7.64-7.62 (m, 2H), 7.40-7.33 (m, 3H), 7.25-7.21 (m, 1H), 7.13-7.09 (m, 2H), 7.06-7.02 (m, 2H), 6.90-6.88 (m, 1H), $6.78(\mathrm{t}, J=$ $2.0 \mathrm{~Hz}, 1 \mathrm{H}), 6.51\left(\mathrm{dd}, J_{1}=8.3 \mathrm{~Hz}, J_{2}=2.3 \mathrm{~Hz}, 1 \mathrm{H}\right), 3.23(\mathrm{~s}, 3 \mathrm{H}) .{ }^{\mathbf{1 3}} \mathbf{C} \mathbf{~ N M R}(\mathbf{1 2 5} \mathbf{M H z}, \mathbf{C D C l})$ $\delta 150.3,145.9,136.4,136.1,130.8,130.3,129.7,128.73,128.70,128.1,128.04,127.96,123.3$, 120.7, 116.3, 112.5, 39.2. HRMS (ESI) calculated $[\mathrm{M}+\mathrm{H}]^{+}$for $\mathrm{C}_{19} \mathrm{H}_{17} \mathrm{BrNSe}: 417.9704$, found: 417.9704. FTIR (cm-1) 3057, 2922, 2363, 1593, 1479, 1340, 1075.

\section{$N$-Methyl-2-(phenylselanyl)- $N$-(3-(trifluoromethyl)phenyl)aniline (4m)}

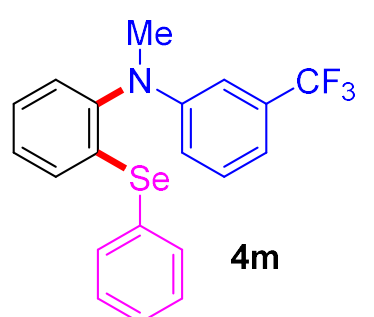

Following the general procedure, treatment of 2-(trimethylsilyl)phenyl trifluoromethanesulfonate 1a $(0.298 \mathrm{~g}, 243 \mu \mathrm{L}, 1.0 \mathrm{mmol})$ and $N, N$ dimethyl-3-(trifluoromethyl)aniline $\mathbf{2 m} \quad(0.095 \mathrm{~g}, 0.5 \quad \mathrm{mmol}) \quad$ with Phenylselenyl bromide 3a $(0.177 \mathrm{~g}, 0.75 \mathrm{mmol})$ in the presence of KF

$(0.116 \mathrm{~g}, 2.0 \mathrm{mmol})$ and 18-crown-6 $(0.528 \mathrm{~g}, 2.0 \mathrm{mmol})$ in THF (2.0 $\mathrm{mL}$ ) at $25^{\circ} \mathrm{C}$ for $12 \mathrm{~h}$ followed by flash column chromatography (Pet.ether/DCM $=95 / 05$ ) of the crude reaction mixture using silica gel afforded $N$-Methyl-2-(phenylselanyl)- $N$-(3(trifluoromethyl)phenyl)aniline $\mathbf{4 m}$ as a pale yellow oil (0.106 g, 52\% yield).

$\boldsymbol{R}_{\mathbf{f}}\left(\right.$ Pet. ether $/ \mathrm{DCM}=$ 95/05): 0.56; ${ }^{1} \mathbf{H}$ NMR (400 MHz, $\left.\mathbf{C D C l}_{3}\right) \delta 7.65(\mathrm{~d}, J=7.1 \mathrm{~Hz}, 2 \mathrm{H})$, 7.43-7.36 (m, 3H), 7.31-7.26 (m, 2H), 7.18-7.11 (m, 3H), 7.05 (d, J=7.6 Hz, 1H), $6.90(\mathrm{~s}, 1 \mathrm{H})$, $6.75(\mathrm{~d}, J=8.3 \mathrm{~Hz}, 1 \mathrm{H}), 3.31(\mathrm{~s}, 3 \mathrm{H}) .{ }^{13} \mathbf{C}$ NMR (100 MHz, $\left.\mathbf{C D C l}_{3}\right) \delta 149.2,145.9,136.4,136.0$, $131.4(\mathrm{q}, J=31.7 \mathrm{~Hz}), 131.0,129.7,129.4,128.7,128.6,128.2,128.1,124.6$ (q, $J=273.6 \mathrm{~Hz})$, $116.9,114.3$ (q, $J=3.8 \mathrm{~Hz}), 109.6(\mathrm{q}, J=3.8 \mathrm{~Hz}), 39.2$. HRMS (ESI) calculated $[\mathrm{M}+\mathrm{H}]^{+}$for $\mathrm{C}_{20} \mathrm{H}_{17} \mathrm{~F}_{3} \mathrm{NSe}: 408.0473$, found: 408.0479. FTIR ( $\left.\mathbf{c m}^{-1}\right)$ 3060, 2922, 1612, 1480, 1354, 1166 , $1122,1073$.

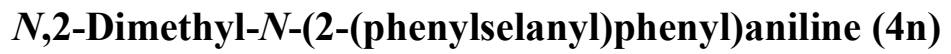

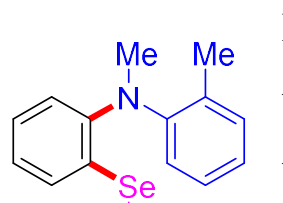

Following the general procedure, treatment of 2-(trimethylsilyl)phenyl trifluoromethanesulfonate 1a $(0.298 \mathrm{~g}, 243 \mu \mathrm{L}, 1.0 \mathrm{mmol})$ and $N, N, 2-$ trimethylaniline 2n (0.068 g, $0.5 \mathrm{mmol})$ with Phenylselenyl bromide 3a $(0.177$ 
$\mathrm{g}, 0.75 \mathrm{mmol})$ in the presence of $\mathrm{KF}(0.116 \mathrm{~g}, 2.0 \mathrm{mmol})$ and 18 -crown-6 $(0.528 \mathrm{~g}, 2.0 \mathrm{mmol})$ in THF $(2.0 \mathrm{~mL})$ at $25{ }^{\circ} \mathrm{C}$ for $12 \mathrm{~h}$ followed by flash column chromatography (Pet.ether/DCM = 95/05) of the crude reaction mixture using silica gel afforded N,2-dimethyl- $N$-(2(phenylselanyl)phenyl)aniline $4 \mathbf{n}$ as a light yellow oil (0.054 g, 31\% yield).

$\boldsymbol{R}_{\mathbf{f}}($ Pet. ether $/ \mathrm{DCM}=95 / 05)$ : 0.47; ${ }^{1} \mathbf{H}$ NMR (400 MHz, $\left.\mathbf{C D C l}_{3}\right) \delta$ 7.63-7.61 (m, 2H), 7.40-7.32 (m, 3H), 7.2-7.14 (m, 2H), 7.10-7.05 (m, 2H), 7.02-6.99 (m, 1H), 6.94-6.92 (m, 1H), 6.89-6.84 (m, 2H), 3.22 (s, 3H), 2.10 (s, 3H). ${ }^{13} \mathbf{C}$ NMR (100 MHz, CDCl 3$) \delta 149.4,136.3,132.7,131.8$, 131.6, 130.6, 129.5, 129.2, 128.3, 126.8, 126.5, 124.5, 123.5, 123.0, 120.7, 41.6, 19.1. HRMS (ESI) calculated $[\mathrm{M}+\mathrm{H}]^{+}$for $\mathrm{C}_{20} \mathrm{H}_{20} \mathrm{NSe}: 354.0755$, found: 354.0760 . FTIR (cm $\left.{ }^{-1}\right)$ 2363, 1636, 1572, 1467, 1278, 1023.

\section{3,4-Difluoro- $N$-methyl- $N$-(2-(phenylselanyl)phenyl)aniline (4o)}<smiles>CN(c1ccc(F)c(F)c1)c1ccccc1[Se]c1ccccc1</smiles>

Following the general procedure, treatment of 2-(trimethylsilyl)phenyl trifluoromethanesulfonate 1a $(0.298 \mathrm{~g}, 243 \mu \mathrm{L}, 1.0 \mathrm{mmol})$ and 3,4difluoro- $N, N$-dimethylaniline $20(0.079 \mathrm{~g}, 0.5 \mathrm{mmol})$ with Phenylselenyl bromide 3a $(0.177 \mathrm{~g}, 0.75 \mathrm{mmol})$ in the presence of $\mathrm{KF}(0.116 \mathrm{~g}, 2.0$ mmol) and 18-crown-6 (0.528 g, $2.0 \mathrm{mmol})$ in THF $(2.0 \mathrm{~mL})$ at $25^{\circ} \mathrm{C}$ for $12 \mathrm{~h}$ followed by flash column chromatography (Pet.ether/DCM $=95 / 05$ ) of the crude reaction mixture using silica gel afforded 3,4-difluoro- $N$-methyl- $N$-(2-(phenylselanyl)phenyl)aniline $\mathbf{4 o}$ as a pale yellow oil ( $0.109 \mathrm{~g}, 58 \%$ yield).

$\boldsymbol{R}_{\mathbf{f}}\left(\right.$ Pet. ether $/ \mathrm{DCM}=$ 95/05): 0.55; ${ }^{1} \mathbf{H}$ NMR (400 MHz, CDCl $) \delta 7.68(\mathrm{~d}, J=7.6 \mathrm{~Hz}, 2 \mathrm{H})$, 7.46-7.39 (m, 3H), 7.30-7.26 (m, 1H), 7.18-7.09 (m, 3H), 7.03 (q, $J=9.4 \mathrm{~Hz}, 1 \mathrm{H}), 6.49-6.44$ (m, 1H), 7.35-7.32 (m, 1H), $3.26(\mathrm{~s}, 3 \mathrm{H}) .{ }^{13} \mathbf{C}$ NMR (100 MHz, CDCl$) \delta 150.7\left(\mathrm{dd}, J_{1}=244.4\right.$ $\left.\mathrm{Hz}, J_{2}=13.4 \mathrm{~Hz}\right), 146.3,146.1,143.5\left(\mathrm{dd}, J_{1}=237.6 \mathrm{~Hz}, J_{2}=13.0 \mathrm{~Hz}\right), 136.4,136.0,130.7$, 129.7, 128.8, 128.5, 128.0, 127.9, $117.2\left(\mathrm{~d}, J_{l}=17.7 \mathrm{~Hz}\right), 108.9\left(\mathrm{dd}, J_{l}=5.1 \mathrm{~Hz}, J_{2}=2.9 \mathrm{~Hz}\right)$, $102.8\left(\mathrm{~d}, J_{1}=21.4 \mathrm{~Hz}\right), 39.5$. HRMS (ESI) calculated $[\mathrm{M}+\mathrm{H}]{ }^{+}$for $\mathrm{C}_{19} \mathrm{H}_{16} \mathrm{~F}_{2} \mathrm{NSe}$ : 376.0411, found: 376.0419. FTIR ( cm$\left.^{-1}\right)$ 3057, 2921, 1600, 1515, 1474, 1269. 


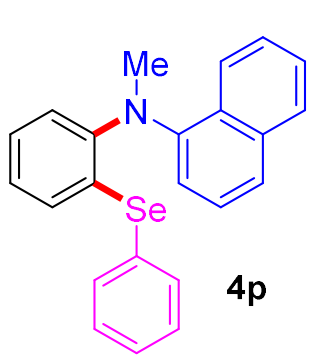

Following the general procedure, treatment of 2-(trimethylsilyl)phenyl trifluoromethanesulfonate 1a $(0.298 \mathrm{~g}, 243 \mu \mathrm{L}, 1.0 \mathrm{mmol})$ and $N, N$ dimethylnaphthalen-1-amine $\quad 2 p\left(\begin{array}{llllll}0.086 & \mathrm{~g}, & 82 & \mu \mathrm{L} 0.5 & \mathrm{mmol}\end{array}\right)$ with Phenylselenyl bromide 3a $(0.177 \mathrm{~g}, 0.75 \mathrm{mmol})$ in the presence of KF $(0.116 \mathrm{~g}, 2.0 \mathrm{mmol})$ and 18 -crown-6 $(0.528 \mathrm{~g}, 2.0 \mathrm{mmol})$ in THF $(2.0 \mathrm{~mL})$ at $25{ }^{\circ} \mathrm{C}$ for $12 \mathrm{~h}$ followed by flash column chromatography (Pet.ether/DCM = 95/05) of the crude reaction mixture using silica gel afforded $\mathrm{N}$-methyl- $\mathrm{N}$-(2(phenylselanyl)phenyl)naphthalen-1-amine $4 p$ as a sticky liquid ( $0.126 \mathrm{~g}, 65 \%$ yield).

$\boldsymbol{R}_{\mathbf{f}}\left(\right.$ Pet. ether /DCM = 95/05): 0.44; ${ }^{1} \mathbf{H}$ NMR (400 MHz, $\left.\mathbf{C D C l}_{3}\right) \delta 8.19(\mathrm{~d}, J=7.4 \mathrm{~Hz}, 1 \mathrm{H}), 7.86$ (d, $J=8.5 \mathrm{~Hz}, 1 \mathrm{H}), 7.88-7.63(\mathrm{~m}, 3 \mathrm{H}), 7.49-7.36(\mathrm{~m}, 6 \mathrm{H}), 7.17(\mathrm{~d}, J=7.4 \mathrm{~Hz}, 1 \mathrm{H}), 7.12-7.04$ (m, 2H), 7.00 (d, $J=7.8 \mathrm{~Hz}, 1 \mathrm{H}), 6.93(\mathrm{t}, J=7.4 \mathrm{~Hz}, 1 \mathrm{H}), 3.42$ (s, 3H). ${ }^{13} \mathbf{C}$ NMR (100 MHz, $\left.\mathbf{C D C l}_{3}\right) \delta 150.4,147.6,136.2,135.2,131.5,131.0,129.6,129.3,129.2,128.4,128.3,127.1$, 125.9, 125.7, 125.6, 125.0, 124.9, 124.1, 123.4, 117.8, 42.8. HRMS (ESI) calculated $[\mathrm{M}+\mathrm{H}]^{+}$ for $\mathrm{C}_{23} \mathrm{H}_{19} \mathrm{NSe}: 390.0755$, found: 390.0760. FTIR ( $\left.\mathbf{c m}^{-1}\right)$ 3052, 2854, 1572, 1466, 1394, 1294, 1022.

\section{Ethyl (E)-3-(4-(methyl(2-(phenylselanyl)phenyl)amino)phenyl)acrylate (4q)}

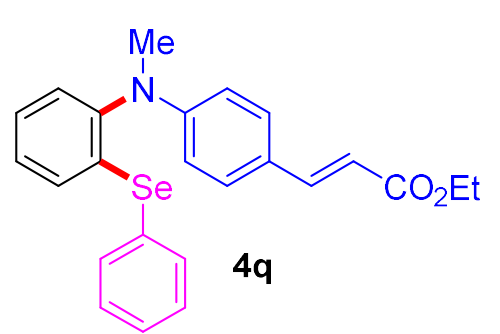

Following the general procedure, treatment of 2(trimethylsilyl)phenyltrifluoromethanesulfonate 1a $(0.149 \mathrm{~g}, 121$ $\mu \mathrm{L}, \quad 0.5 \mathrm{mmol}$ ) and ethyl (E)-3-(4-(dimethylamino)phenyl) acrylate $2 \mathbf{q}(0.055 \mathrm{~g}, 0.25 \mathrm{mmol})$ with Phenylselenyl bromide 3a $(0.088 \mathrm{~g}, 0.375 \mathrm{mmol})$ in the presence of $\mathrm{KF}(0.058 \mathrm{~g}, 1.0 \mathrm{mmol})$ and 18-crown-6 $(0.264 \mathrm{~g}, 1.0 \mathrm{mmol})$ in THF $(1.0 \mathrm{~mL})$ at $65{ }^{\circ} \mathrm{C}$ for $12 \mathrm{~h}$ followed by flash column chromatography (Pet.ether/DCM $=80 / 20$ ) of the crude reaction mixture using silica gel afforded ethyl (E)-3-(4-(methyl(2-(phenylselanyl)phenyl)amino)phenyl)acrylate $\mathbf{4 q}$ as a pale yellow oil ( $0.055 \mathrm{~g}, 50 \%$ yield).

$\boldsymbol{R}_{\mathbf{f}}\left(\right.$ Pet. ether /DCM = 80/20): 0.30; ${ }^{1} \mathbf{H}$ NMR (400 MHz, $\left.\mathbf{C D C l}_{\mathbf{3}}\right) \delta$ 7.65-7.59 (m, 3H), 7.39-7.32 (m, 5H), 7.26-7.22 (m, 1H), 7.15-7.10 (m, 2H), 7.07-7.05 (m, 1H), $6.57(\mathrm{~d}, J=8.7 \mathrm{~Hz}, 2 \mathrm{H}), 6.23$ ( d, $J=15.9 \mathrm{~Hz}, 1 \mathrm{H}), 4.24$ (q, $J=7.1 \mathrm{~Hz}, 2 \mathrm{H}), 3.28$ (s, 3H), 1.33 (t, $J=7.1 \mathrm{~Hz}, 3 \mathrm{H}) .{ }^{13} \mathbf{C}$ NMR $\left(100 \mathrm{MHz}, \mathbf{C D C l}_{3}\right) \delta 167.9,150.6,145.6,145.1,136.4,136.0,130.9,129.7,129.6,128.83$, 128.76, 128.2, 128.02, 128.00, 124.0, 113.5, 113.4, 60.3, 39.1, 14.5. HRMS (ESI) calculated 
$[\mathrm{M}+\mathrm{H}]^{+}$for $\mathrm{C}_{24} \mathrm{H}_{24} \mathrm{NO}_{2} \mathrm{Se}: 438.0967$, found: 438.0970. FTIR $\left(\mathbf{c m}^{-1}\right)$ 3060, 2922, 2329, 1702 , $1624,1171,1038$.

\section{$N$-Methyl- $N$-phenyl-2-(p-tolylselanyl)aniline (4r)}

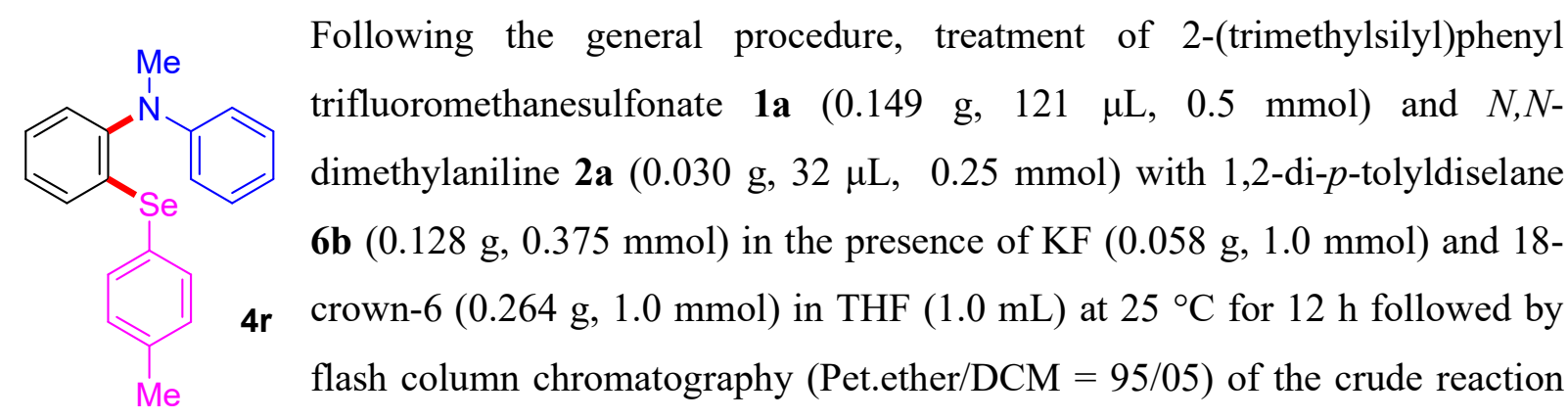
mixture using silica gel afforded $N$-methyl- $N$-phenyl-2-( $p$-tolylselanyl)aniline $\mathbf{4 r}$ as a pale yellow solid $(0.049 \mathrm{~g}, 56 \%$ yield $)$.

$\boldsymbol{R}_{\mathbf{f}}\left(\right.$ Pet. ether $/ \mathrm{DCM}=$ 95/05): 0.45; ${ }^{1} \mathbf{H}$ NMR (400 MHz, CDCl$) \delta 7.53(\mathrm{~d}, J=8.0 \mathrm{~Hz}, 2 \mathrm{H})$, 7.24-7.17 (m, 5H), 7.13-7.11 (m, 1H), 7.08-7.04 (m, 1H), 6.98-6.93 (m, 1H), 6.79 (t, $J=7.3 \mathrm{~Hz}$, 1H), $6.66(\mathrm{~d}, J=8.3 \mathrm{~Hz}, 2 \mathrm{H}), 3.25$ (s, 3H), 2.39 (s, 3H). ${ }^{13} \mathbf{C}$ NMR (100 MHz, CDCl$) \delta 149.2$, 146.6, 138.9, 136.93, 136.88, 130.6, 129.8, 129.1, 128.5, 127.5, 127.4, 124.3, 118.0, 114.0, 39.2, 21.4. HRMS (ESI) calculated $[\mathrm{M}+\mathrm{H}]^{+}$for $\mathrm{C}_{20} \mathrm{H}_{20} \mathrm{NSe}: 354.0755$, found: 354.0760 . FTIR (cm 1) $3058,2920,1642,1601,1497,1337$.

\section{2-((4-Bromophenyl)selanyl)- $N$-methyl- $N$-phenylaniline (4s)}<smiles>CN(c1ccccc1)c1ccccc1[Se]c1ccc(Br)cc1</smiles>

Following the general procedure, treatment of 2-(trimethylsilyl)phenyl trifluoromethanesulfonate $1 \mathrm{a}(0.149 \mathrm{~g}, 121 \mu \mathrm{L}, 0.5 \mathrm{mmol})$ and $\mathrm{N}, \mathrm{N}$ dimethylaniline 2a $(0.030 \mathrm{~g}, 32 \mu \mathrm{L}, \quad 0.25 \mathrm{mmol})$ with 1,2-bis(4bromophenyl $)$ diselane $6 \mathbf{c}(0.176 \mathrm{~g}, 0.375 \mathrm{mmol})$ in the presence of $\mathrm{KF}(0.058$

$4 s \mathrm{~g}, 1.0 \mathrm{mmol})$ and 18 -crown-6 (0.264 g, $1.0 \mathrm{mmol})$ in THF $(1.0 \mathrm{~mL})$ at $25^{\circ} \mathrm{C}$ for $12 \mathrm{~h}$ followed by flash column chromatography (Pet.ether/DCM =95/05) of the crude reaction mixture using silica gel afforded 2-((4bromophenyl)selanyl)- $N$-methyl- $N$-phenylaniline $4 \mathrm{~s}$ as a light yellow solid (0.058g, 56\% yield). $\boldsymbol{R}_{\mathbf{f}}$ (Pet. ether /DCM = 95/05): 0.50; ${ }^{1} \mathbf{H}$ NMR (400 MHz, $\left.\mathbf{C D C l}_{3}\right) \delta$ 7.50-7.45 (m, 4H), 7.26-7.20 (m, 3H), 7.17-7.15 (m, 1H), 7.12-7.08 (m, 1H), 7.03-7.01 (m, 1H), $6.80(\mathrm{t}, J=7.3 \mathrm{~Hz}, 1 \mathrm{H}), 6.64$ (d, $J=7.9 \mathrm{~Hz}, 2 \mathrm{H}), 3.24$ (s, 3H). ${ }^{13} \mathbf{C}$ NMR (100 MHz, CDCl$) \delta 149.1,147.0,138.0,135.7$, 
$132.8,130.4,129.1,128.7,128.0,127.7,127.4,123.2,118.2,114.1,39.3$. HRMS (ESI) calculated $[\mathrm{M}+\mathrm{H}]{ }^{+}$for $\mathrm{C}_{19} \mathrm{H}_{17} \mathrm{BrNSe}: 417.9704$, found: 417.9713. FTIR (cm $\left.{ }^{-1}\right)$ 3056, 2921, 1600, 1497, 1465, 1006.

\section{2-((4-Chlorophenyl)selanyl)- $N$-methyl- $N$-phenylaniline (4t)}

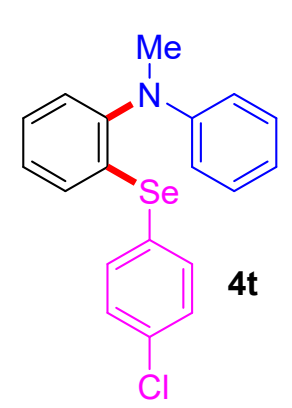

Following the general procedure, treatment of 2-(trimethylsilyl)phenyl trifluoromethanesulfonate $1 \mathrm{a}(0.149 \mathrm{~g}, 121 \mu \mathrm{L}, 0.5 \mathrm{mmol})$ and $N, N$ dimethylaniline $\mathbf{2 a}(0.030 \mathrm{~g}, 32 \mu \mathrm{L}, 0.25 \mathrm{mmol})$ with 1,2-bis(4-chlorophenyl) diselane $6 \mathbf{d}(0.143 \mathrm{~g}, 0.375 \mathrm{mmol})$ in the presence of $\mathrm{KF}(0.058 \mathrm{~g}, 1.0 \mathrm{mmol})$ and 18-crown-6 (0.264 g, $1.0 \mathrm{mmol})$ in THF $(1.0 \mathrm{~mL})$ at $25{ }^{\circ} \mathrm{C}$ for $12 \mathrm{~h}$ followed by flash column chromatography (Pet.ether/DCM $=95 / 05)$ of the crude reaction mixture using silica gel afforded 2-((4-chlorophenyl)selanyl)- $N$-methyl- $N$ phenylaniline $4 \mathrm{t}$ as a light yellow solid $(0.059 \mathrm{~g}, 63 \%$ yield $)$.

$\boldsymbol{R}_{\mathbf{f}}\left(\right.$ Pet. ether /DCM = 95/05): 0.53; ${ }^{1} \mathbf{H}$ NMR (400 MHz, $\left.\mathbf{C D C l}_{3}\right) \delta$ 7.57-7.54 (m, 2H), 7.34-7.31 (m, 2H), 7.26-7.20 (m, 3H), 7.17-7.15 (m, 1H), 7.12-7.08 (m, 1H), $7.01\left(\mathrm{dd}, J_{l}=7.9 \mathrm{~Hz}, J_{2}=1.5\right.$ $\mathrm{Hz}, 1 \mathrm{H}), 6.83-6.79(\mathrm{~m}, 1 \mathrm{H}), 6.66-6.64(\mathrm{~m}, 2 \mathrm{H}), 3.25$ (s, 3H) ${ }^{13} \mathbf{C}$ NMR (100 MHz, CDCl 3$) \delta$ 149.1, 147.0, 137.8, 135.8, 135.1, 130.3, 129.9, 129.1, 128.7, 128.0, 127.7, 126.7, 118.2, 114.1, 39.3. HRMS (ESI) calculated $[\mathrm{M}+\mathrm{H}]^{+}$for $\mathrm{C}_{19} \mathrm{H}_{17} \mathrm{ClNSe}: 374.0209$, found: 374.0211 . FTIR (cm 1) $3057,2363,1600,1498,1338,1087$.

\section{2-((4-Fluorophenyl)selanyl)- $N$-methyl- $N$-phenylaniline (4u)}<smiles>CN(c1ccccc1)c1ccccc1[Se]c1ccc(F)cc1</smiles>

Following the general procedure, treatment of 2-(trimethylsilyl)phenyl trifluoromethanesulfonate $1 \mathrm{a}(0.149 \mathrm{~g}, 121 \mu \mathrm{L}, 0.5 \mathrm{mmol})$ and $N, N-$ dimethylaniline $2 \mathrm{a}(0.030 \mathrm{~g}, \quad 32 \mu \mathrm{L}, \quad 0.25 \mathrm{mmol})$ with 1,2-bis(4fluorophenyl)diselane $6 \mathbf{e}(0.131 \mathrm{~g}, 0.375 \mathrm{mmol})$ in the presence of $\mathrm{KF}(0.058$ $\mathrm{g}, 1.0 \mathrm{mmol})$ and 18 -crown-6 $(0.264 \mathrm{~g}, 1.0 \mathrm{mmol})$ in THF $(1.0 \mathrm{~mL})$ at $25^{\circ} \mathrm{C}$ for $12 \mathrm{~h}$ followed by flash column chromatography (Pet.ether/DCM =95/05) of the crude reaction mixture using silica gel afforded 2-((4-fluorophenyl)selanyl)- $N$-methyl- $N$ phenylaniline $4 \mathbf{u}$ as a yellow solid $(0.063 \mathrm{~g}, 71 \%$ yield $)$.

$\boldsymbol{R}_{\mathbf{f}}$ (Pet. ether /DCM = 95/05): 0.50; ${ }^{1} \mathbf{H}$ NMR (400 MHz, $\left.\mathbf{C D C l}_{3}\right) \delta$ 7.68-7.63 (m, 2H), 7.29-7.23 (m, 3H), $7.18\left(\mathrm{dd}, J_{1}=7.8 \mathrm{~Hz}, J_{2}=1.5 \mathrm{~Hz}, 1 \mathrm{H}\right), 7.14-7.08(\mathrm{~m}, 3 \mathrm{H}), 6.98\left(\mathrm{~d}, J=7.8 \mathrm{~Hz}, J_{2}=1.5\right.$ 
Hz, 1H), 6.84 (t, $J=7.3 \mathrm{~Hz}, 1 \mathrm{H}), 6.71-6.68(\mathrm{~m}, 2 \mathrm{H}), 3.29$ (s, 3H). ${ }^{13} \mathbf{C}$ NMR (100 MHz, CDCl 3$)$ $\delta 163.4(\mathrm{~d}, J=248.6 \mathrm{~Hz}), 149.1,146.7,138.9$ (d, $J=8.0 \mathrm{~Hz}), 137.4(\mathrm{~d}, J=8.1 \mathrm{~Hz}), 136.4,129.8$, 129.1, 128.6, 127.7 (d, $J=9.4 \mathrm{~Hz}), 122.8$ (d, $J=3.4 \mathrm{~Hz}), 118.2,116.9$ (d, $J=21.4 \mathrm{~Hz}), 114.1$, 39.2. HRMS (ESI) calculated $[\mathrm{M}+\mathrm{H}]^{+}$for $\mathrm{C}_{19} \mathrm{H}_{17} \mathrm{FNSe}$ : 358.0505, found: 358.0512 . FTIR (cm 1) $3060,2363,1641,1582,1489,1226$.

\section{2-((3-Chlorophenyl)selanyl)- $N$-methyl- $N$-phenylaniline (4v)}<smiles>CN(c1ccccc1)c1ccccc1[Se]c1cccc(Cl)c1</smiles>

Following the general procedure, treatment of 2-(trimethylsilyl)phenyl trifluoromethanesulfonate $1 \mathrm{a}(0.149 \mathrm{~g}, 121 \mu \mathrm{L}, 0.5 \mathrm{mmol})$ and $N, N$ dimethylaniline 2a $(0.030 \mathrm{~g}, 32 \mu \mathrm{L}, \quad 0.25 \mathrm{mmol})$ with 1,2-bis(3chlorophenyl $)$ diselane $\mathbf{6 f}(0.143 \mathrm{~g}, 0.375 \mathrm{mmol})$ in the presence of KF $(0.058$ $\mathrm{g}, 1.0 \mathrm{mmol})$ and 18 -crown-6 $(0.264 \mathrm{~g}, 1.0 \mathrm{mmol})$ in THF $(1.0 \mathrm{~mL})$ at $25^{\circ} \mathrm{C}$ for $12 \mathrm{~h}$ followed by flash column chromatography (Pet.ether/DCM =95/05) of the crude reaction mixture using silica gel afforded 2-((3-chlorophenyl)selanyl)- $N$-methyl- $N$ phenylaniline $4 \mathbf{v}$ as a white solid ( $0.035 \mathrm{~g}, 38 \%$ yield).

$\boldsymbol{R}_{\mathbf{f}}$ (Pet. ether /DCM = 95/05): 0.53; ${ }^{1} \mathbf{H}$ NMR (400 MHz, $\left.\mathbf{C D C l}_{3}\right) \delta$ 7.61-7.57 (m, 1H), 7.49-7.47 (m, 1H), 7.35-7.32 (m, 1H), 7.28-7.09 (m, 6H), 7.06-7.04 (m, 1H), $6.79(\mathrm{t}, J=7.3 \mathrm{~Hz}, 1 \mathrm{H}), 6.62$ $(\mathrm{d}, J=7.9 \mathrm{~Hz}, 2 \mathrm{H}), 3.23$ (s, 3H). ${ }^{13} \mathbf{C}$ NMR (100 MHz, $\left.\mathbf{C D C l}_{3}\right) \delta 149.0,147.1,135.7,135.3$, $135.0,134.1,130.8,130.6,130.2,129.0,128.68,128.67,128.2,127.7,118.2,114.0,39.3$. HRMS (ESI) calculated $[\mathrm{M}+\mathrm{H}]{ }^{+}$for $\mathrm{C}_{19} \mathrm{H}_{17} \mathrm{ClNSe}: 374.0209$, found: 374.0214 . FTIR (cm 1) $3057,2921,1600,1567,1497,1461,1027$.

\section{2-((2-Chlorophenyl)selanyl)- $N$-methyl- $N$-phenylaniline (4w)}<smiles>CN(c1ccccc1)c1ccccc1[Se]c1ccccc1Cl</smiles>

Following the general procedure, treatment of 2-(trimethylsilyl)phenyl trifluoromethanesulfonate $1 \mathrm{a}(0.149 \mathrm{~g}, 121 \mu \mathrm{L}, 0.5 \mathrm{mmol})$ and $N, N$ dimethylaniline $\mathbf{2 a}(0.030 \mathrm{~g}, \quad 32 \mu \mathrm{L}, \quad 0.25 \mathrm{mmol})$ with 1,2-bis(2chlorophenyl)diselane $\mathbf{6 g}(0.143 \mathrm{~g}, 0.375 \mathrm{mmol})$ in the presence of $\mathrm{KF}$ $(0.058 \mathrm{~g}, 1.0 \mathrm{mmol})$ and 18 -crown-6 $(0.264 \mathrm{~g}, 1.0 \mathrm{mmol})$ in THF $(1.0 \mathrm{~mL})$ at $25{ }^{\circ} \mathrm{C}$ for $12 \mathrm{~h}$ followed by flash column chromatography (Pet.ether/DCM $=95 / 05$ ) of the crude reaction mixture using silica gel afforded 2-((2-chlorophenyl)selanyl)- $N$-methyl- $N$-phenylaniline 4w as a pale yellow oil ( $0.046 \mathrm{~g}, 49 \%$ yield). 
$\boldsymbol{R}_{\mathbf{f}}\left(\right.$ Pet. ether $/ \mathrm{DCM}=$ 95/05): 0.51; ${ }^{1} \mathbf{H}$ NMR (400 MHz, CDCl $) \delta 7.55\left(\mathrm{dd}, J_{1}=7.7 \mathrm{~Hz}, J_{2}=\right.$ $1.3 \mathrm{~Hz}, 1 \mathrm{H}), 7.47\left(\mathrm{dd}, J_{1}=8.0 \mathrm{~Hz}, J_{2}=1.1 \mathrm{~Hz}, 1 \mathrm{H}\right), 7.30-7.25(\mathrm{~m}, 2 \mathrm{H}), 7.24-7.17(\mathrm{~m}, 4 \mathrm{H}), 7.16-$ $7.09(\mathrm{~m}, 2 \mathrm{H}), 6.79(\mathrm{t}, J=7.3 \mathrm{~Hz}, 1 \mathrm{H}), 6.67-6.65(\mathrm{~m}, 2 \mathrm{H}), 3.25(\mathrm{~s}, 3 \mathrm{H}) .{ }^{13} \mathbf{C}$ NMR (100 MHz, CDCl$\left._{3}\right) \delta 149.2,148.1,138.5,136.9,133.9,132.1,130.1,129.7,129.1,128.9,128.7,127.7$, 127.5, 118.2, 114.1, 39.4. HRMS (ESI) calculated $[\mathrm{M}+\mathrm{H}]^{+}$for $\mathrm{C}_{19} \mathrm{H}_{17} \mathrm{ClNSe}: 374.0209$, found: 374.0210. FTIR (cm-1) 3057, 2922, 1598, 1496, 1445, 1022.

\section{$N, 4,5-T r i m e t h y l-N$-phenyl-2-(phenylselanyl)aniline (4x)}<smiles>COc1cc([Se]c2ccccc2)c(N(C)c2ccccc2)cc1C</smiles>

Following the general procedure, treatment of 4,5-dimethyl-2(trimethylsilyl)phenyl trifluoromethanesulfonate $\mathbf{1 b}(0.326 \mathrm{~g}, 1.0 \mathrm{mmol})$ and $N, N$-dimethylaniline 2a $(0.061 \mathrm{~g}, 63 \mu \mathrm{L}, 0.5 \mathrm{mmol})$ with Phenylselenyl bromide 3a $(0.177 \mathrm{~g}, 0.75 \mathrm{mmol})$ in the presence of $\mathrm{KF}$ $(0.116 \mathrm{~g}, 2.0 \mathrm{mmol})$ and 18-crown-6 (0.528 g, $2.0 \mathrm{mmol})$ in THF (2.0 $\mathrm{mL}$ ) at $25^{\circ} \mathrm{C}$ for $12 \mathrm{~h}$ followed by flash column chromatography (Pet.ether/DCM $=95 / 05$ ) of the crude reaction mixture using silica gel afforded $N, 4,5$-trimethyl- $N$-phenyl-2(phenylselanyl)aniline $4 \mathbf{x}$ as a white solid ( $0.124 \mathrm{~g}, 68 \%$ yield).

$\boldsymbol{R}_{\mathbf{f}}\left(\right.$ Pet. ether $/ \mathrm{DCM}=$ 95/05): 0.45; ${ }^{1} \mathbf{H}$ NMR (400 MHz, CDCl3) $\delta$ 7.61-7.59(m, 2H), 7.367.31(m, 3H), 7.24-7.20 (m, 2H), $6.97(\mathrm{~s}, 1 \mathrm{H}), 6.91(\mathrm{~s}, 1 \mathrm{H}), 6.78(\mathrm{t}, J=7.3 \mathrm{~Hz}, 1 \mathrm{H}), 6.64(\mathrm{~d}, J=$ 7.9 Hz, 2H),3.22 (s, 3H), 2.20 (s, 3H), 2.16 (s, 3H). ${ }^{13} \mathbf{C}$ NMR (125 MHz, CDCl $) \delta$ 149.4, $145.1,136.9,136.3,135.6,132.4,131.6,129.7,129.4,129.0,128.1,117.6,113.7,39.3,19.5$, 19.4. HRMS (ESI) calculated $[\mathrm{M}+\mathrm{H}]{ }^{+}$for $\mathrm{C}_{21} \mathrm{H}_{22} \mathrm{NSe}$ : 368.0912, found: 368.0919 . FTIR (cm1) $3059,2919,2363,1597,1495,1380,1328,1025$.

\section{$N$-Methyl- $N$-phenyl-6-(phenylselanyl)-2,3-dihydro-1 $H$-inden-5-amine (4y)}<smiles>CN(c1ccccc1)c1cc2c(cc1[Se]c1ccccc1)CCC2</smiles>

Following the general procedure, treatment of 6-(trimethylsilyl)-2,3dihydro- $1 H$-inden-5-yl trifluoromethanesulfonate $1 \mathrm{c}(0.338 \mathrm{~g}, 1.0 \mathrm{mmol})$ and $N, N$-dimethylaniline $2 \mathrm{a}(0.061 \mathrm{~g}, 63 \mu \mathrm{L}, \quad 0.5 \mathrm{mmol})$ with Phenylselenyl bromide 3a $(0.177 \mathrm{~g}, 0.75 \mathrm{mmol})$ in the presence of KF

4y (0.116 g, $2.0 \mathrm{mmol})$ and 18-crown-6 (0.528 g, $2.0 \mathrm{mmol})$ in THF (2.0 $\mathrm{mL}$ ) at $25{ }^{\circ} \mathrm{C}$ for $12 \mathrm{~h}$ followed by flash column chromatography (Pet.ether/DCM $=95 / 05$ ) of the crude reaction mixture using silica gel afforded $N$-methyl- $N$ - 
phenyl-6-(phenylselanyl)-2,3-dihydro- $1 H$-inden-5-amine $4 \mathbf{y}$ as a white solid $(0.150 \mathrm{~g}, 79 \%$ yield).

$\boldsymbol{R}_{\mathbf{f}}$ (Pet. ether /DCM = 95/05): 0.40; ${ }^{1} \mathbf{H}$ NMR (400 MHz, $\left.\mathbf{C D C l}_{3}\right) \delta$ 7.68-7.65 (m, 2H), 7.42-7.35 $(\mathrm{m}, 3 \mathrm{H}), 7.28-7.23(\mathrm{~m}, 2 \mathrm{H}), 7.06(\mathrm{~s}, 1 \mathrm{H}), 6.97(\mathrm{~s}, 1 \mathrm{H}), 6.81\left(\mathrm{tt}, J_{l}=7.4 \mathrm{~Hz}, J_{2}=1.0 \mathrm{~Hz}, 1 \mathrm{H}\right)$, 6.71-6.68 (m. 2H), 3.26 (s, 3H), 2.88-2.80 (m, 4H), 2.09 (p, $J=7.4 \mathrm{~Hz}, 2 \mathrm{H}) .{ }^{13} \mathbf{C}$ NMR (100 MHz, CDCl3) $\delta 149.5,145.3,144.7,144.1,136.0,132.9,129.5,129.2,129.0,128.2,126.5$, 124.4, 117.6, 113.8, 39.3, 32.7, 32.6, 25.8. HRMS (ESI)calculated $[\mathrm{M}+\mathrm{H}]^{+}$for $\mathrm{C}_{22} \mathrm{H}_{22} \mathrm{NSe}$ : 380.0912, found: 380.0915. FTIR (cm $\left.{ }^{-1}\right)$ 3588, 3062, 3951, 2843, 1598, 1499.

\section{4,5-Difluoro- $N$-methyl- $N$-phenyl-2-(phenylselanyl)aniline (4z)}<smiles>CN(c1ccccc1)c1cc(F)c(F)cc1[Se]c1ccccc1</smiles>

Following the general procedure, treatment of 4,5-difluoro-2(trimethylsilyl)phenyl trifluoromethanesulfonate $1 \mathbf{d}(0.334 \mathrm{~g}, 1.0 \mathrm{mmol})$ and $N, N$-dimethylaniline $2 \mathrm{a}(0.061 \mathrm{~g}, \quad 63 \mu \mathrm{L}, \quad 0.5 \mathrm{mmol})$ with Phenylselenyl bromide 3a $(0.177 \mathrm{~g}, 0.75 \mathrm{mmol})$ in the presence of $\mathrm{KF}$

$(0.116 \mathrm{~g}, 2.0 \mathrm{mmol})$ and 18 -crown-6 $(0.528 \mathrm{~g}, 2.0 \mathrm{mmol})$ in THF $(2.0 \mathrm{~mL})$ at $25{ }^{\circ} \mathrm{C}$ for $12 \mathrm{~h}$ followed by flash column chromatography (Pet.ether/DCM $=95 / 05$ ) of the crude reaction mixture using silica gel afforded4,5-difluoro- $N$-methyl- $N$-phenyl-2(phenylselanyl)aniline $\mathbf{4 z}$ as a pale yellow oil ( $0.102 \mathrm{~g}, 55 \%$ yield).

$\boldsymbol{R}_{\mathbf{f}}($ Pet. ether $/ \mathrm{DCM}=95 / 05)$ : 0.55; ${ }^{1} \mathbf{H}$ NMR (400 MHz, $\left.\mathbf{C D C l}_{3}\right) \delta$ 7.65-7.63(m, 2H), 7.46$7.38(\mathrm{~m}, 3 \mathrm{H}), 7.28-7.24(\mathrm{~m}, 2 \mathrm{H}), 6.99\left(\mathrm{dd}, J_{1}=10.7 \mathrm{~Hz}, J_{2}=7.4 \mathrm{~Hz}, 1 \mathrm{H}\right), 6.86(\mathrm{t}, J=7.3 \mathrm{~Hz}, 1 \mathrm{H})$, $6.76\left(\mathrm{dd}, J_{1}=10.5 \mathrm{~Hz}, J_{2}=8.6 \mathrm{~Hz}, 1 \mathrm{H}\right), 6.70-6.67(\mathrm{~m}, 2 \mathrm{H}), 3.23(\mathrm{~s}, 3 \mathrm{H}) .{ }^{13} \mathbf{C}$ NMR (100 MHz, $\left.\mathbf{C D C l}_{3}\right) \delta 149.7\left(\mathrm{dd}, J_{l}=254.1 \mathrm{~Hz}, J_{2}=16.6 \mathrm{~Hz}\right), 149.4\left(\mathrm{dd}, J_{I}=242.9 \mathrm{~Hz}, J_{2}=7.0 \mathrm{~Hz}\right), 148.7$, $142.6\left(\mathrm{dd}, J_{1}=6.1 \mathrm{~Hz}, J_{2}=3.7 \mathrm{~Hz}\right), 136.7,132.3\left(\mathrm{dd}, J_{1}=4.5 \mathrm{~Hz}, J_{2}=3.5 \mathrm{~Hz}\right), 130.0,129.3$, 129.2, 127.5, 118.9, $118.0(\mathrm{~d}, J=19.7 \mathrm{~Hz}), 117.1\left(\mathrm{dd}, J_{1}=16.3 \mathrm{~Hz}, J_{2}=1.2 \mathrm{~Hz}\right), 114.4,39.3$. HRMS (ESI) calculated $[\mathrm{M}+\mathrm{H}]{ }^{+}$for $\mathrm{C}_{19} \mathrm{H}_{16} \mathrm{~F}_{2} \mathrm{NSe}$ : 376.0411, found: 376.0417. FTIR (cm1) $3061,2877,1597,1488,1392,1286,1170$.

\section{$N$-Methyl- $N$-phenyl-1-(phenylselanyl)naphthalen-2-amine (4aa)}<smiles>CN(c1ccccc1)c1ccc2ccccc2c1[Se]c1ccccc1</smiles>

Following the general procedure, treatment of 3-(trimethylsilyl) naphthalen-2-yl trifluoromethanesulfonate 1e $(0.174 \mathrm{~g}, 0.5 \mathrm{mmol})$ and $N, N$-dimethylaniline $2 \mathbf{a}(0.030 \mathrm{~g}, 32 \mu \mathrm{L}, 0.25 \mathrm{mmol})$ with Phenylselenyl 
bromide 3a $(0.088 \mathrm{~g}, 0.375 \mathrm{mmol})$ in the presence of KF $(0.058 \mathrm{~g}, 1.0 \mathrm{mmol})$ and 18 -crown-6 $(0.264 \mathrm{~g}, 1.0 \mathrm{mmol})$ in THF $(1.0 \mathrm{~mL})$ at $25{ }^{\circ} \mathrm{C}$ for $12 \mathrm{~h}$ followed by flash column chromatography (Pet.ether/DCM $=95 / 05$ ) of the crude reaction mixture using silica gel afforded $N$-methyl- $N$-phenyl-1-(phenylselanyl)naphthalen-2-amine 4aa as a yellow oil $(0.047 \mathrm{~g}, 48 \%$ yield).

$\boldsymbol{R}_{\mathbf{f}}\left(\right.$ Pet. ether $/ \mathrm{DCM}=$ 95/05): 0.3; ${ }^{1} \mathbf{H}$ NMR (400 MHz, CDCl $)$ ) $\delta$ 8.47-8.45(m, 1H), 7.96 (d, $J=$ 8.6 Hz, 1H), 7.88-7.86 (m, 1H), 7.52-7.46 (m, 2H), 7.42 (d, $J=8.6 \mathrm{~Hz}, 1 \mathrm{H}), 7.17-7.06(\mathrm{~m}, 7 \mathrm{H})$, $7.73(\mathrm{t}, J=7.3 \mathrm{~Hz}, 1 \mathrm{H}), 6.55(\mathrm{~d}, J=7.9 \mathrm{~Hz}, 2 \mathrm{H}), 3.24$ (s, 3H). ${ }^{13} \mathbf{C}$ NMR (100 MHz, CDCl $) \delta$ $150.2,149.2,136.4,133.4,132.9,132.0,130.1,129.4,129.1,129.0,128.5,127.5,127.4,126.2$, 126.0, 117.5, 113.6, 39.9. HRMS (ESI) calculated $[\mathrm{M}+\mathrm{H}]^{+}$for $\mathrm{C}_{23} \mathrm{H}_{20} \mathrm{NSe}$ : 390.0755, found: 390.0764. FTIR (cm-1) 3056, 2923, 2364, 1589, 1497, 1362, 1131, 1023.

\section{$N$-Methyl- $N$-phenyl-1-(phenylselanyl)-5,6,7,8-tetrahydronaphthalen-2-amine (4ab)}

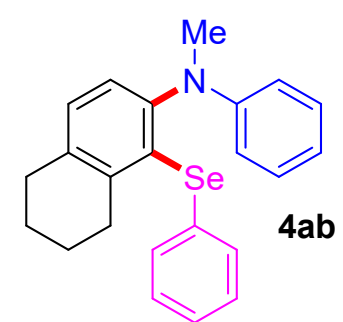

Following the general procedure, treatment of 1-(trimethylsilyl)-5,6,7,8tetrahydronaphthalen-2-yl trifluoromethanesulfonate $\mathbf{1 f}(0.352 \mathrm{~g}, 1.0$ mmol) and $N, N$-dimethylaniline $2 \mathrm{a}(0.061 \mathrm{~g}, 63 \mu \mathrm{L}, 0.5 \mathrm{mmol})$ with Phenylselenyl bromide 3a $(0.177 \mathrm{~g}, 0.75 \mathrm{mmol})$ in the presence of KF $(0.116 \mathrm{~g}, 2.0 \mathrm{mmol})$ and 18 -crown-6 $(0.528 \mathrm{~g}, 2.0 \mathrm{mmol})$ in THF $(2.0 \mathrm{~mL})$

at $25{ }^{\circ} \mathrm{C}$ for $12 \mathrm{~h}$ followed by flash column chromatography (Pet.ether/DCM $=95 / 05$ ) of the crude reaction mixture using silica gel afforded $N$-methyl- $N$-phenyl-1-(phenylselanyl)-5,6,7,8tetrahydronaphthalen-2-amine $\mathbf{4 a b}$ as a pale yellow oil ( $0.134 \mathrm{~g}, 68 \%$ yield).

$\boldsymbol{R}_{\mathbf{f}}\left(\right.$ Pet. ether $/ \mathrm{DCM}=$ 95/05): 0.4; ${ }^{1} \mathbf{H}$ NMR (400 MHz, $\left.\mathbf{C D C l}_{3}\right) \delta 7.22(\mathrm{~d}, J=8.1 \mathrm{~Hz}, 1 \mathrm{H})$, 7.18-7.10(m, 8H), 6.70 (t, $J=7.2 \mathrm{~Hz}, 1 \mathrm{H}), 6.50(\mathrm{~d}, J=8.1 \mathrm{~Hz}, 2 \mathrm{H}), 3.13(\mathrm{~s}, 3 \mathrm{H}), 2.84$ (d, $J=6.7$ Hz, 4H), 1.76 (t, $J=2.8 \mathrm{~Hz}, 4 \mathrm{H}) .{ }^{13} \mathbf{C}$ NMR (125 MHz, CDCl3) $\delta$ 149.7, 149.3, 143.5, 137.2, 133.4, 132.5, 132.0, 129.8, 129.1, 128.9, 126.7, 125.8, 116.7, 112.9, 39.7, 31.1, 30.2, 23.6, 22.8. HRMS (ESI) calculated $[\mathrm{M}+\mathrm{H}]{ }^{+}$for $\mathrm{C}_{23} \mathrm{H}_{24} \mathrm{NSe}$ : 394.1068, found: 394.1075. FTIR (cm ${ }^{-}$ 1) 3059, 2934, 2861, 1602, 1501, 1347.

\section{$N, 4-d i m e t h y l-N$-phenyl-2-(phenylselanyl)aniline (4ac) and $N, 5$-dimethyl- $N$-phenyl-2- (phenylselanyl)aniline (4ac')}


<smiles>Cc1ccc(N(C)c2ccccc2)c([Se]c2ccccc2)c1</smiles>

mmol $)$ and 18-crown-6 $(0.264 \mathrm{~g}, 1.0 \mathrm{mmol})$ in THF $(1.0 \mathrm{~mL})$ at $25^{\circ} \mathrm{C}$ for $12 \mathrm{~h}$ followed by flash column chromatography (Pet.ether/DCM $=95 / 05$ ) of the crude reaction mixture using silica gel afforded $N, 4$-dimethyl- $N$-phenyl-2-(phenylselanyl)aniline (4ac) and $N, 5$-dimethyl- $N$-phenyl-2(phenylselanyl)aniline (4ac') as a mixture of regioisomers in 1.4:1 ratio as a pale yellow oil (0.065 g,74\% yield).

$\boldsymbol{R}_{\mathbf{f}}\left(\right.$ Pet. ether /DCM = 95/05): 0.49; ${ }^{1} \mathbf{H}$ NMR (400 MHz, $\left.\mathbf{C D C l}_{\mathbf{3}}\right)$ of Major isomer; $\delta$ 7.69-7.64 (m, 2H), 7.45-7.35 (m, 3H), 7.28-7.24 (m, 2H), 7.09-7.00 (m, 2H), 6.96-6.94 (m, 1H), 6.85-6.80 (m, 1H), 6.71-6.68 (m. 2H), 3.27 (s, 3H), 2.32 (s, 3H). ${ }^{1} \mathbf{H}$ NMR (400 MHz, CDCl $)$ of Minor isomer; $\delta$ 7.69-7.64 $(\mathrm{m}, 2 \mathrm{H}), 7.45-7.35(\mathrm{~m}, 3 \mathrm{H}), 7.28-7.24(\mathrm{~m}, 2 \mathrm{H}), 7.09-7.00(\mathrm{~m}, 2 \mathrm{H}), 6.89(\mathrm{~s}$, 1H), 6.85-6.80 (m, 1H), 6.71-6.68 (m. 2H), 3.27 (s, 3H), 2.26 (s, 3H). ${ }^{13}$ C NMR (125 MHz, $\mathbf{C D C l}_{3}$ ) of Major isomer $\delta$ 149.2, 147.1, 138.1, 135.9, 131.9, 131.0, 129.5, 129.2, 129.1, 129.0, 128.5, 128.3, 117.9, 113.9, 39.3, 20.9. ${ }^{\mathbf{1 3}} \mathbf{C}$ NMR (125 MHz, CDCl $)$ of Major isomer $\delta$ 149.1, $144.5,137.5,136.3,135.7,130.9,129.6,129.0,128.7,128.4,128.5,128.3,117.8,113.8,39.2$, 21.2. HRMS (ESI)calculated $[\mathrm{M}+\mathrm{H}]{ }^{+}$for $\mathrm{C}_{20} \mathrm{H}_{20} \mathrm{NSe}: 354.0755$, found:354.0757. FTIR (cm 1) $3058,2919,1599,1495,1335,1032$.

\section{4-Chloro- $N$-methyl- $N$-phenyl-2-(phenylselanyl)aniline (4ad) and 5-Chloro- $N$-methyl- $N$ - phenyl-2-(phenylselanyl)aniline(4ad')}

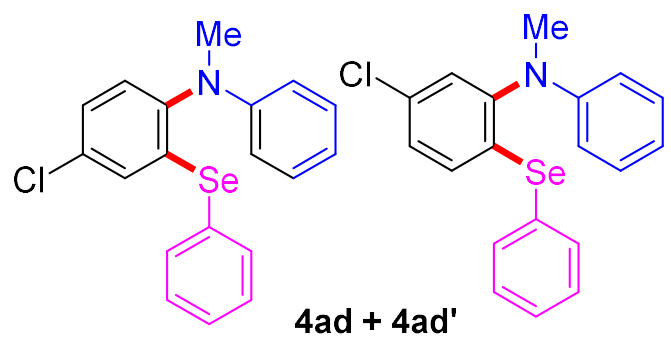

Following the general procedure, treatment of 4chloro-2-(trimethylsilyl)phenyl trifluoro methanesulfonate $\mathbf{1 h}(0.333 \mathrm{~g}, 1.0 \mathrm{mmol}) \mathrm{N}, N-$ dimethylaniline 2a $(0.061 \mathrm{~g}, 63 \mu \mathrm{L}, 0.5 \mathrm{mmol})$ and with Phenylselenyl bromide 3a $(0.177 \mathrm{~g}, 0.75 \mathrm{mmol})$ in the presence of KF $(0.116 \mathrm{~g}, 2.0 \mathrm{mmol})$ and 18 -crown-6 $(0.528 \mathrm{~g}, 2.0 \mathrm{mmol})$ in THF $(2.0 \mathrm{~mL})$ at25 ${ }^{\circ} \mathrm{C}$ for $12 \mathrm{~h}$ followed by flash column chromatography (Pet.ether/DCM $=95 / 05$ ) of the crude reaction mixture using silica gel afforded 4-chloro- $N$-methyl- $N$-phenyl-2- 
(phenylselanyl)aniline (4ad) and 5-chloro- $N$-methyl- $N$-phenyl-2-(phenylselanyl)aniline (4ad') as as a mixture of regioisomers in 1.7:1 ratio $(0.118 \mathrm{~g}, 68 \%$ yield, pale yellow oil, regioisomer ratio determined by ${ }^{1} \mathrm{H}-\mathrm{NMR}$ analysis of crude reaction mixture).

$\boldsymbol{R}_{\mathbf{f}}$ (Pet. ether $/ \mathrm{DCM}=$ 95/05): 0.53; ${ }^{1} \mathbf{H}$ NMR (400 MHz, CDCl $) \delta$ 7.71-7.66(m, 3H), 7.507.38(m, 4H), 7.31-7.27 (m, 3H), 6.75-6.72(m, 3H), $3.27(\mathrm{~s}, 3 \mathrm{H})$. Representative peaks of other isomer 1H NMR (400 MHz, CDCl 3$) \delta 3.28$ (s). ${ }^{13} \mathbf{C}$ NMR (100 MHz, CDCl $) \delta 148.8,145.2$, 138.6, 136.8, 133.1, 131.4, 129.9, 129.4, 129.14, 129.10, 128.8, 127.6, 118.5, 114.2, 39.2. Representative peaks of other isomer ${ }^{13} \mathbf{C}$ NMR (125 MHz, $\left.\mathbf{C D C l}_{3}\right) \delta 148.6,147.9,136.4$, $132.8,132.7,129.8,129.3,129.2,128.5,128.0,127.5,127.3,118.8,114.6,39.2$. HRMS (ESI) calculated $[\mathrm{M}+\mathrm{H}]^{+}$for $\mathrm{C}_{19} \mathrm{H}_{17} \mathrm{ClNSe}: 374.0209$, found: 374.0210. FTIR (cm $\left.{ }^{-1}\right)$ 3061, 2950, 2871, 1601, 1499, 1332.

4-Fluoro- $N$-methyl- $N$-phenyl-2-(phenylselanyl)aniline(4ae) and 5-fluoro- $N$-methyl- $N$ phenyl-2-(phenylselanyl)aniline (4ae')<smiles>CN(c1ccccc1)c1cc(F)ccc1[Se]c1ccccc1</smiles>

Following the general procedure, treatment of 5 fluoro-2-(trimethylsilyl)phenyl trifluoro methanesulfonate $1 \mathbf{i}(0.316 \mathrm{~g}, 1.0 \mathrm{mmol})$ and $N, N$ dimethylaniline $2 \mathrm{a}(0.061 \mathrm{~g}, 63 \mu \mathrm{L}, 0.5 \mathrm{mmol})$ with Phenylselenyl bromide 3a $(0.177 \mathrm{~g}, 0.75 \mathrm{mmol})$ in the presence of KF (0.116 g, $2.0 \mathrm{mmol})$ and 18-crown-6 $(0.528 \mathrm{~g}, 2.0 \mathrm{mmol})$ in THF $(2.0 \mathrm{~mL})$ at $25{ }^{\circ} \mathrm{C}$ for $12 \mathrm{~h}$ followed by flash column chromatography (Pet.ether/DCM $=95 / 05$ ) of the crude reaction mixture using silica gel afforded 4-fluoro- $N$-methyl- $N$-phenyl-2-(phenylselanyl)aniline (4ae) and 5-fluoro- $N$-methyl- $N$-phenyl-2(phenylselanyl)aniline (4ae') as a mixture of regioisomers in 2.3:1 ratio as a sticky liquid (0.100 g, $56 \%$ yield).

$\boldsymbol{R}_{\mathbf{f}}\left(\right.$ Pet. ether $/ \mathrm{DCM}=$ 95/05): 0.50; ${ }^{1} \mathbf{H}$ NMR (400 MHz, $\left.\mathbf{C D C l}_{3}\right) \delta$ 7.70-7.68 (m, 2H), 7.49-7.40 (m, 3H), 7.28-7.24 (m, 2H), 7.11-7.07 (m, 1H), 6.87-6.82 (m, 2H), 6.72-6.68 (m, 2H), 6.64 (dd, $\left.J_{1}=9.0 \mathrm{~Hz}, J_{2}=2.9 \mathrm{~Hz}, 1 \mathrm{H}\right), 3.25(\mathrm{~s}, 3 \mathrm{H})$. Representative peaks of other isomer ${ }^{1} \mathbf{H}$ NMR (400 MHz, CDCl 3$) \delta 3.26$ (s). ${ }^{13}$ C NMR (125 MHz, CDCl $) \delta 161.7$ (d, $\left.J=248.1 \mathrm{~Hz}\right), 149.1,142.4$ (d, $J=2.9 \mathrm{~Hz}), 139.3$ (d, $J=7.6 \mathrm{~Hz}), 137.0,132.3$ (d, $J=8.9 \mathrm{~Hz}), 129.9,129.2,129.1,127.3$, $118.3,116.1(\mathrm{~d}, J=24.9 \mathrm{~Hz}), 114.7(\mathrm{~d}, J=21.4 \mathrm{~Hz}), 113.9,39.2$. Representative peaks of other isomer ${ }^{13} \mathbf{C}$ NMR (125 MHz, $\left.\mathbf{C D C l}_{3}\right) \delta 162.7(\mathrm{~d}, J=247.7 \mathrm{~Hz}), 148.8,148.6(\mathrm{~d}, J=8.4$ 
Hz), 135.9, 129.7, 129.6, 129.1, 128.9, 128.5, $118.8,115.4$ (d, $J=21.0 \mathrm{~Hz}), 114.2$ (d, $J=23.0$ $\mathrm{Hz}), 114.6,39.4$. HRMS (ESI) calculated $[\mathrm{M}+\mathrm{H}]^{+}$for $\mathrm{C}_{19} \mathrm{H}_{17} \mathrm{FNSe}: 358.0505$, found: 358.0508 . FTIR (cm $\left.{ }^{-1}\right)$ 3061, 2871, 1596, 1477, 1252, 1194.

\section{$N, N$-Dimethyl-2-(phenylselanyl)aniline (4af)}

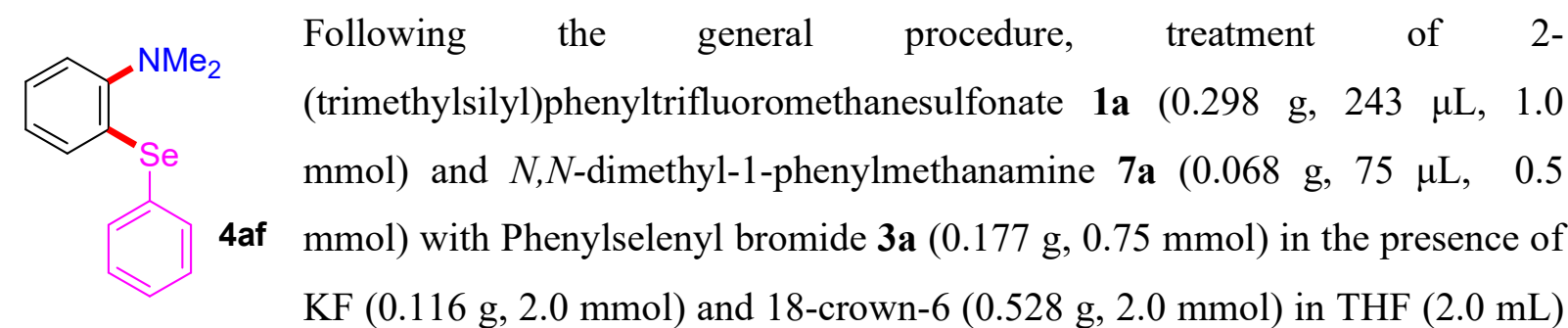
at $25{ }^{\circ} \mathrm{C}$ for $12 \mathrm{~h}$ followed by flash column chromatography (Pet.ether/DCM $=95 / 05$ ) of the crude reaction mixture using silica gel afforded $N, N$-dimethyl-2-(phenylselanyl)aniline 4af as a white solid ( $0.064 \mathrm{~g}, 46 \%$ yield).

$\boldsymbol{R}_{\mathbf{f}}\left(\right.$ Pet. ether /DCM = 95/05): 0.31; ${ }^{1} \mathbf{H}$ NMR (400 MHz, CDCl $) \delta$ 7.69-7.67 (m, 2H), 7.43-7.36 (m, 3H), 7.18-7.14 (m, 2H), 6.91-6.84 (m, 2H), 2.80 (s, 6H). ${ }^{13} \mathbf{C}$ NMR (100 MHz, CDCl$) \delta$ $151.9,136.7,132.3,129.6,129.3,128.8,128.5,126.6,125.0,120.2,45.1$. HRMS (ESI) calculated $[\mathrm{M}+\mathrm{H}]{ }^{+}$for $\mathrm{C}_{14} \mathrm{H}_{16} \mathrm{NSe}$ : 278.0442, found: 278.0446. FTIR ( $\left.\mathbf{c m}^{-1}\right)$ 3049, 2828, 2784 , $2363,1573,1470,1438,941$.

\section{1-(2-(Phenylselanyl)phenyl)-1,2,3,4-tetrahydroquinoline (4ag)}<smiles>c1ccc([Se]c2ccccc2N2CCCc3ccccc32)cc1</smiles>

Following the general procedure, treatment of 2-(trimethylsilyl) phenyltrifluoromethanesulfonate $1 \mathbf{a}(0.298 \mathrm{~g}, 243 \mu \mathrm{L}, 1.0 \mathrm{mmol})$ and 1methyl-1,2,3,4-tetrahydroquinoline $7 \mathrm{c} \quad(0.074 \mathrm{~g}, 0.5 \mathrm{mmol})$ with Phenylselenyl bromide $3 \mathbf{a}(0.177 \mathrm{~g}, 0.75 \mathrm{mmol})$ in the presence of KF $(0.116$

4ag g, $2.0 \mathrm{mmol})$ and 18-crown-6 $(0.528 \mathrm{~g}, 2.0 \mathrm{mmol})$ in THF $(2.0 \mathrm{~mL})$ at $25^{\circ} \mathrm{C}$ for $12 \mathrm{~h}$ followed by flash column chromatography (Pet.ether/DCM =95/05) of the crude reaction mixture using silica gel afforded 1-(2-(phenylselanyl)phenyl)-1,2,3,4tetrahydroquinoline $4 \mathbf{a g}$ as a colorless oil ( $0.081 \mathrm{~g}, 44 \%$ yield $)$.

$\boldsymbol{R}_{\mathbf{f}}($ Pet. ether $/ \mathrm{DCM}=95 / 05)$ : 0.40; ${ }^{1} \mathbf{H}$ NMR (400 MHz, $\left.\mathbf{C D C l}_{3}\right) \delta$ 7.66-7.63(m, 2H), 7.397.36(m, 3H), 7.23-7.21 (m, 2H), 7.09-7.03(m, 2H), $6.99(\mathrm{~d}, J=7.7 \mathrm{~Hz}, 1 \mathrm{H}), 6.91(\mathrm{t}, J=7.3 \mathrm{~Hz}$, $1 \mathrm{H}), 6.66(\mathrm{t}, J=7.4 \mathrm{~Hz}, 1 \mathrm{H}), 6.20(\mathrm{~d}, J=8.1 \mathrm{~Hz}, 1 \mathrm{H}), 3.61-3.50(\mathrm{~m}, 2 \mathrm{H}), 2.91(\mathrm{~s}, 2 \mathrm{H}), 2.18-2.13$ 
(m, 2H). ${ }^{13} \mathbf{C}$ NMR (100 MHz, CDCl 3$) \delta 145.8,145.1,136.8,136.7,120.0,129.7,129.5,128.9$, 128.7, 128.3 127.7, 127.6, 126.8, 122.7, 117.7, 114.2, 50.8, 28.0, 22.7. HRMS (ESI) calculated $[\mathrm{M}+\mathrm{H}]{ }^{+}$for $\mathrm{C}_{21} \mathrm{H}_{20} \mathrm{NSe}: 366.0755$, found: 366.0760. FTIR (cm-1) 3450, 3060, 2924, 1573, $1495,1304$.

\section{$N$-Methyl- $N$-phenyl-2-(phenylthio)aniline (9a)}

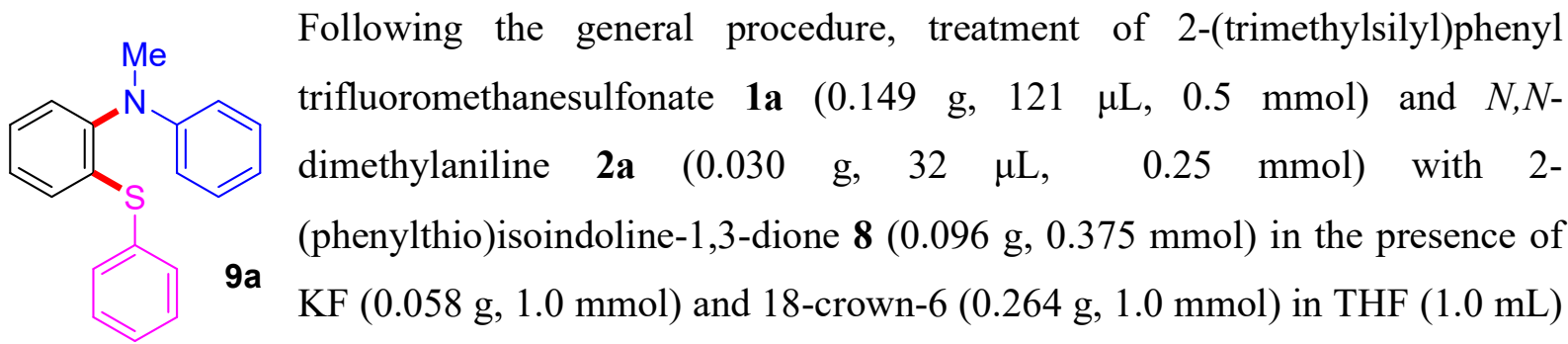
at $25{ }^{\circ} \mathrm{C}$ for $12 \mathrm{~h}$ followed by flash column chromatography (Pet.ether/DCM = 95/05) of the crude reaction mixture using silica gel afforded $N$-methyl- $N$-phenyl-2-(phenylthio)aniline 9a as a white solid (0.029 g, 40\% yield).

$\boldsymbol{R} \mathbf{f}\left(\right.$ Pet. ether $\left./ \mathrm{CH}_{2} \mathrm{Cl}_{2}=95 / 05\right)$ : 0.46; ${ }^{1} \mathbf{H}$ NMR (400 MHz, $\left.\mathbf{C D C l}_{3}\right) \delta$ 7.48-7.44 (m, 2H), 7.39$7.31(\mathrm{~m}, 3 \mathrm{H}), 7.24-7.19(\mathrm{~m}, 4 \mathrm{H}), 7.14-7.11(\mathrm{~m}, 1 \mathrm{H}), 7.03-7.01(\mathrm{~m}, 1 \mathrm{H}), 6.78(\mathrm{t}, J=7.3 \mathrm{~Hz}, 1 \mathrm{H})$, 6.65-6.63 (m, 2H), 3.25 (s, 3H). ${ }^{13}$ C NMR (100 MHz, CDCl $) \delta 149.0,146.0,138.8,134.1$, 133.4, 129.5, 129.9, 128.9, 128.2, 127.3, 127.2, 117.7, 113.6, 39.1. HRMS (ESI) calculated $[\mathrm{M}+\mathrm{H}]^{+}$for $\mathrm{C}_{19} \mathrm{H}_{18} \mathrm{NS}: 292.1154$, found: 292.1160. FTIR $\left(\mathbf{c m}^{-1}\right)$ 3058, 2924, 2874, 2808, 2333, 1919, 1729, 1600, 1577, 1496, 1442, 1340, 1131, 1027, 867.

\section{$N$-(4-Bromobutyl)- $N$-phenyl-2-(phenylselanyl)aniline (13)}

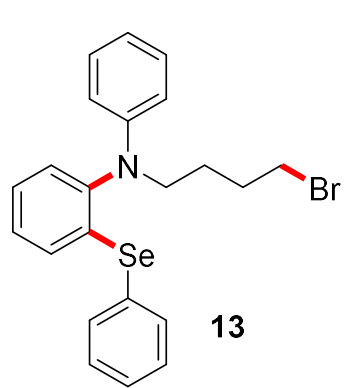

Following the general procedure, treatment of 2(trimethylsilyl)phenyltrifluoromethanesulfonate 1a $(0.298 \mathrm{~g}, 243 \mu \mathrm{L}, 1.0$ mmol) and 1-phenylpyrrolidine $7 \mathbf{d}(0.074 \mathrm{~g}, \quad 0.5 \mathrm{mmol})$ with Phenylselenyl bromide 3a $(0.177 \mathrm{~g}, 0.75 \mathrm{mmol})$ in the presence of KF $(0.116 \mathrm{~g}, 2.0 \mathrm{mmol})$ and 18-crown-6 (0.528 g, $2.0 \mathrm{mmol})$ in THF (2.0 $\mathrm{mL}$ ) at $25{ }^{\circ} \mathrm{C}$ for $12 \mathrm{~h}$ followed by flash column chromatography (Pet.ether/DCM $=95 / 05)$ of the crude reaction mixture using silica gel afforded $\mathrm{N}$-(4bromobutyl)- $N$-phenyl-2-(phenylselanyl)aniline $\mathbf{1 3}$ as a white solid ( $0.080 \mathrm{~g}, 35 \%$ yield). 
$\boldsymbol{R}_{\mathbf{f}}$ (Pet. ether $/ \mathrm{DCM}=$ 95/05): 0.48; ${ }^{1} \mathbf{H}$ NMR (400 MHz, CDCl $) \delta$ 7.69-7.64 (m, 2H), 7.45-7.38 $(\mathrm{m}, 3 \mathrm{H}), 7.30-7.21(\mathrm{~m}, 4 \mathrm{H}), 7.16-7.12(\mathrm{~m}, 1 \mathrm{H}), 7.07\left(\mathrm{dd}, J_{l}=7.9 \mathrm{~Hz}, J_{2}=1.2 \mathrm{~Hz}, 1 \mathrm{H}\right), 6.83(\mathrm{t}, J$ $=7.3 \mathrm{~Hz}, 1 \mathrm{H}), 6.68-6.66(\mathrm{~m}, 2 \mathrm{H}), 3.75-3.71(\mathrm{~m}, 2 \mathrm{H}), 3.49(\mathrm{t}, J=6.5 \mathrm{~Hz}, 2 \mathrm{H}), 2.09-1.92(\mathrm{~m}, 4 \mathrm{H})$. ${ }^{13}$ C NMR (100 MHz, CDCl $) \delta 148.1,144.7,136.9,136.5,130.6,129.9,129.7,129.2,128.7$, 128.3, 127.6, 127.5, 118.0, 114.0, 50.8, 33.5, 30.6, 26.4. HRMS (ESI) calculated $[\mathrm{M}+\mathrm{H}]{ }^{+}$for $\mathrm{C}_{22} \mathrm{H}_{23}$ BrNSe: 460.0174, found: 460.0178. FTIR (cm-1) 3057, 2922, 1599, 1497, 1465, 1027.

\section{$N$-Phenyl-2-(phenylselanyl)- $N$-(4-(phenylselanyl)butyl)aniline (14)}

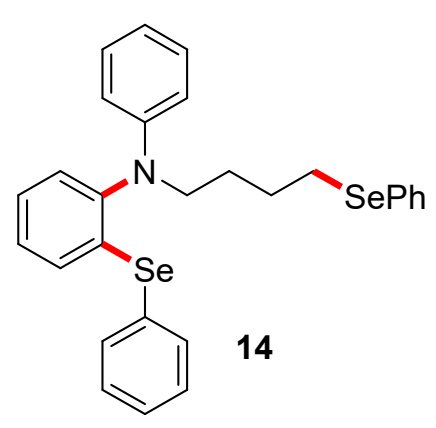

Following the general procedure, treatment of 2(trimethylsilyl)phenyl trifluoromethanesulfonate 1a (0.298 g, 243 $\mu \mathrm{L}, 1.0 \mathrm{mmol})$ and 1-phenylpyrrolidine $7 \mathbf{d}(0.074 \mathrm{~g}, 0.5 \mathrm{mmol})$ with 1,2-diphenyldiselane $6(0.234 \mathrm{~g}, 0.75 \mathrm{mmol})$ in the presence of $\mathrm{KF}$ $(0.116 \mathrm{~g}, 2.0 \mathrm{mmol})$ and 18 -crown-6 $(0.528 \mathrm{~g}, 2.0 \mathrm{mmol})$ in THF $(2.0 \mathrm{~mL})$ at $25{ }^{\circ} \mathrm{C}$ for $12 \mathrm{~h}$ followed by flash column chromatography (Pet.ether/DCM $=95 / 05$ ) of the crude reaction mixture using silica gel afforded $N$-phenyl-2-(phenylselanyl)- $N$-(4-(phenylselanyl)butyl)aniline 14 as a pale yellow oil ( $0.093 \mathrm{~g}, 34 \%$ yield $)$.

$\boldsymbol{R}_{\mathbf{f}}\left(\right.$ Pet. ether /DCM = 95/05): 0.33; ${ }^{1} \mathbf{H}$ NMR (400 MHz, $\left.\mathbf{C D C l}_{3}\right) \delta$ 7.62-7.60 (m, 2H), 7.51-7.49 (m, 2H), 7.39-7.33 (m, 3H), 7.29-7.18 (m, 6H), 7.14-7.12 (m, 1H), 7.10-7.06 (m, 1H), 7.02-7.00 (m, 1H), $6.77(\mathrm{t}, J=7.3 \mathrm{~Hz}, 1 \mathrm{H}), 6.60(\mathrm{~d}, J=7.9 \mathrm{~Hz}, 2 \mathrm{H}), 3.65-3.61(\mathrm{~m}, 2 \mathrm{H}), 2.98-2.92(\mathrm{~m}$, 2H), 1.93-1.85 (m, 2H), 1.83-1.76 (m, 2H). ${ }^{13}$ C NMR (100 MHz, CDCl $) \delta$ 148.1, 144.8, 137.0, 136.6, 132.9, 130.5, 130.4, 130.0, 129.7, 129.2, 128.7, 128.4, 127.6, 127.4, 126.9, 117.8, 114.0, 51.0, 28.0, 27.8. HRMS (ESI) calculated $[\mathrm{M}+\mathrm{H}]^{+}$for $\mathrm{C}_{28} \mathrm{H}_{28} \mathrm{NSe}_{2}$ : 538.0547, found: 538.0555. FTIR (cm-1) 3056, 2922, 1599, 1572, 1496, 1022.

\section{Synthesis and Characterization of 2-Selanyl aniline Derivatives}

$N$-Methyl- $N$-phenyl-2-(phenylselanyl)aniline (4a) 

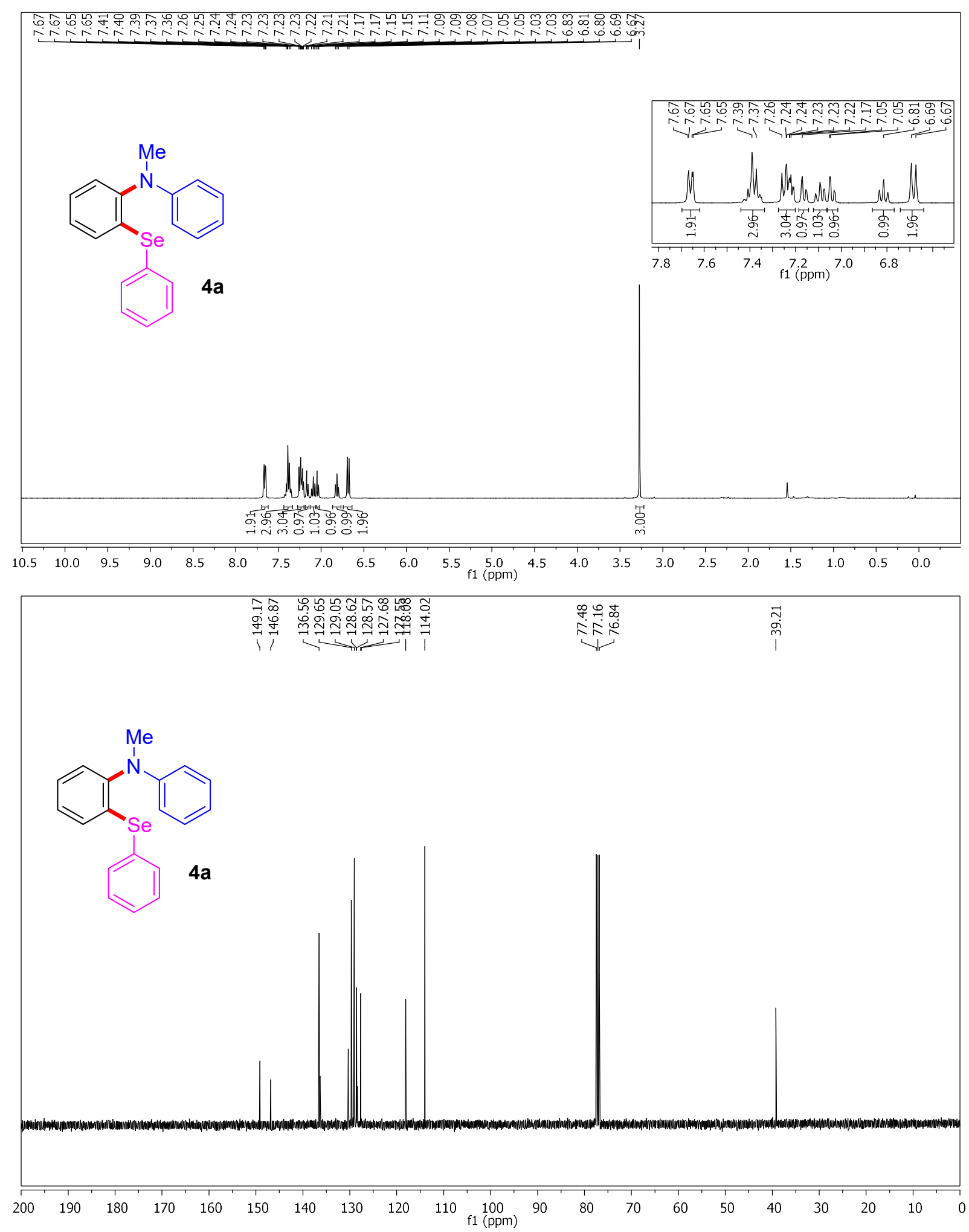

$\mathrm{N}$-(4-Methoxyphenyl)- $\mathrm{N}$-methyl-2-(phenylselanyl)aniline (4b) 

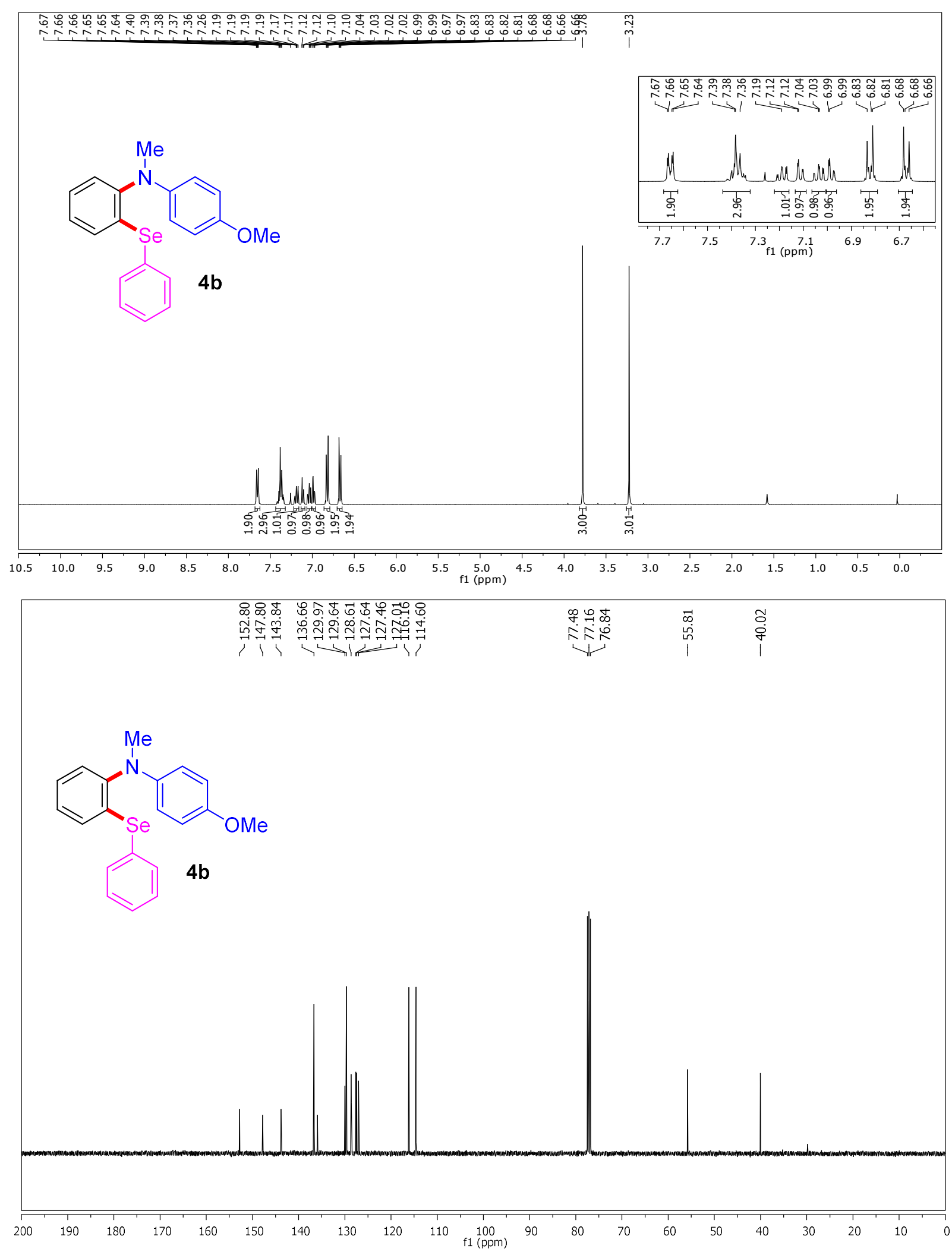

$\mathrm{N}$-Methyl-2-(phenylselanyl)- $\mathrm{N}$-(p-tolyl)aniline (4c) 

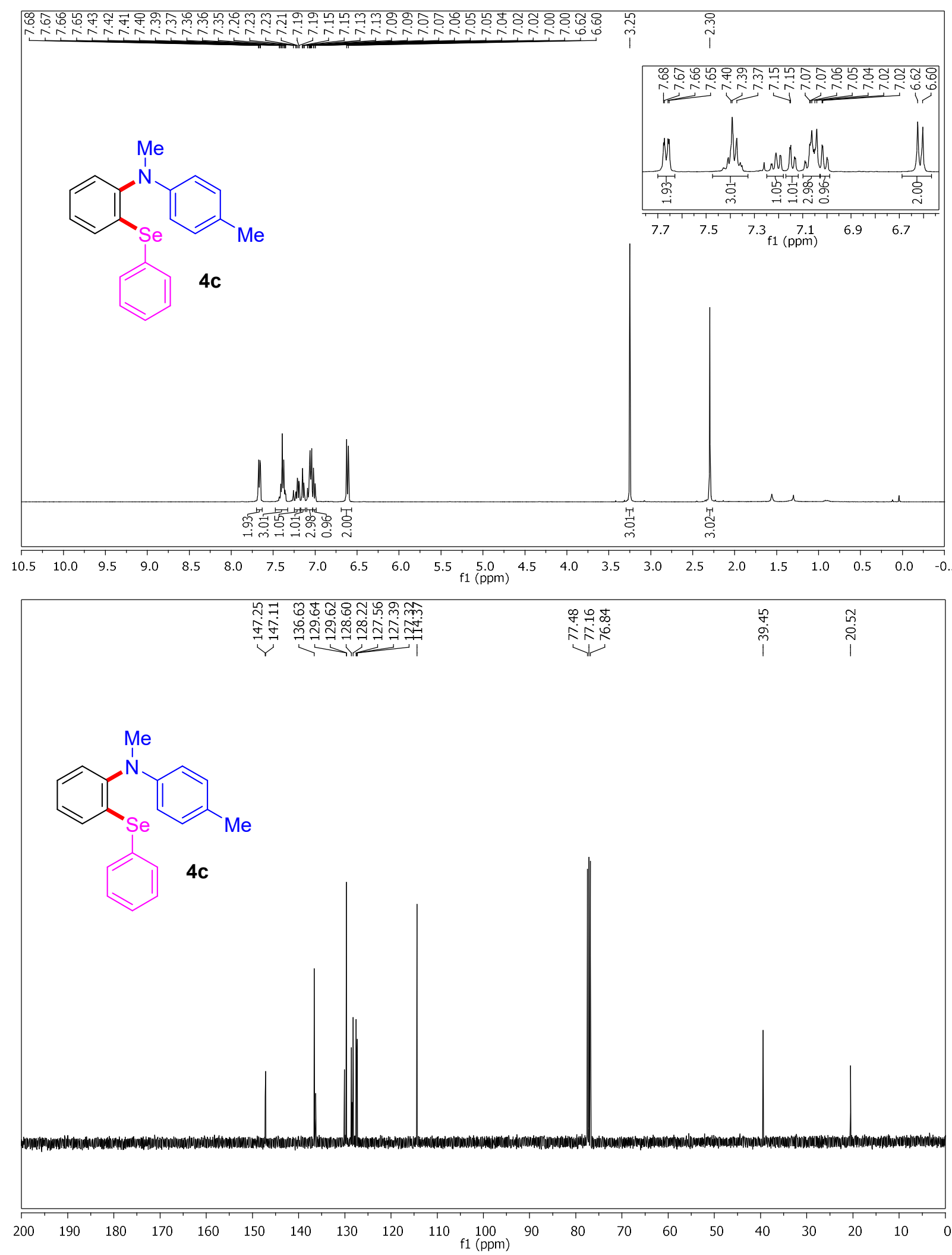

$N$-(4-Ethylphenyl)- $N$-methyl-2-(phenylselanyl)aniline (4d) 

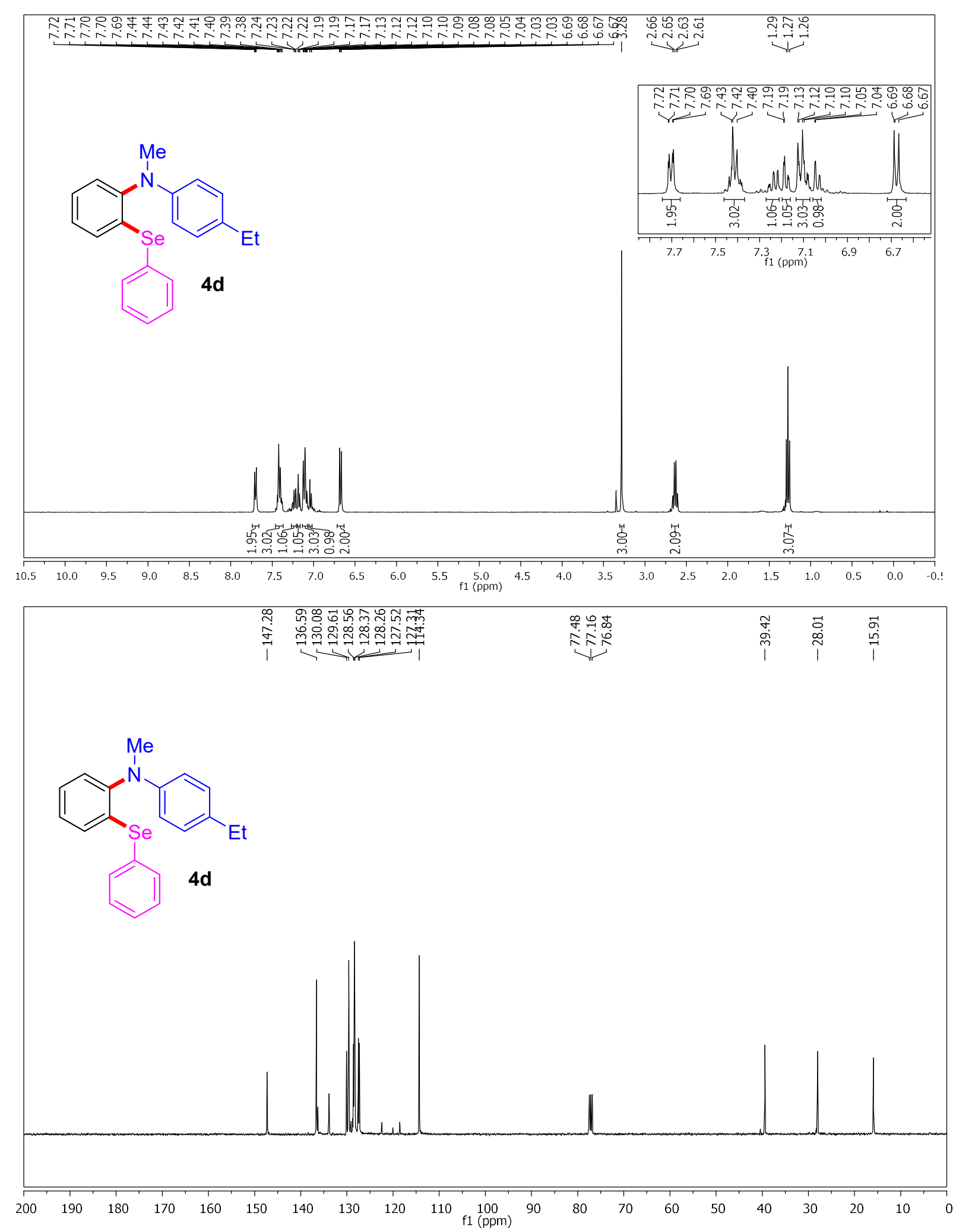

$N$-(4-Iodophenyl)- $N$-methyl-2-(phenylselanyl)aniline (4e) 

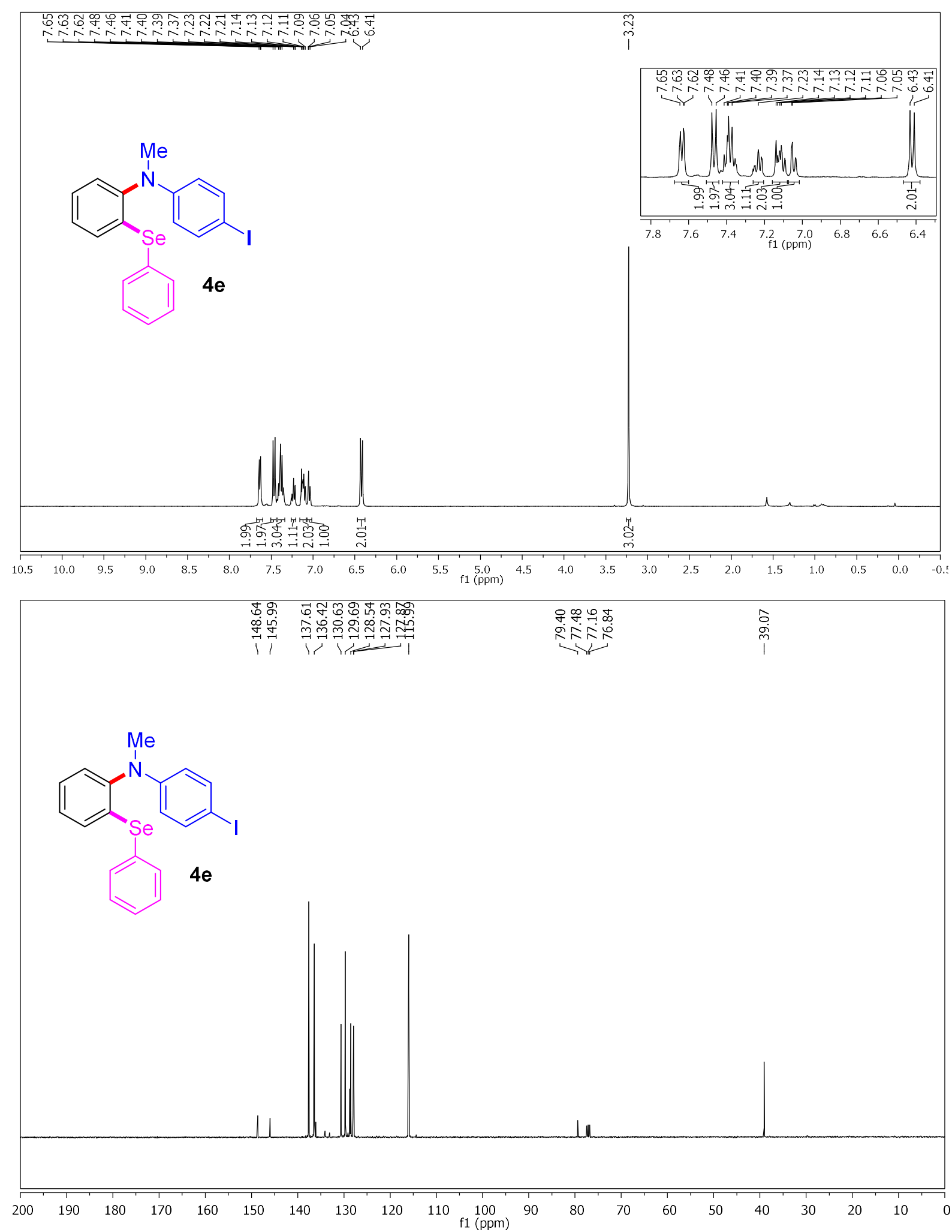

$N$-(4-Bromophenyl)- $N$-methyl-2-(phenylselanyl)aniline (4f) 

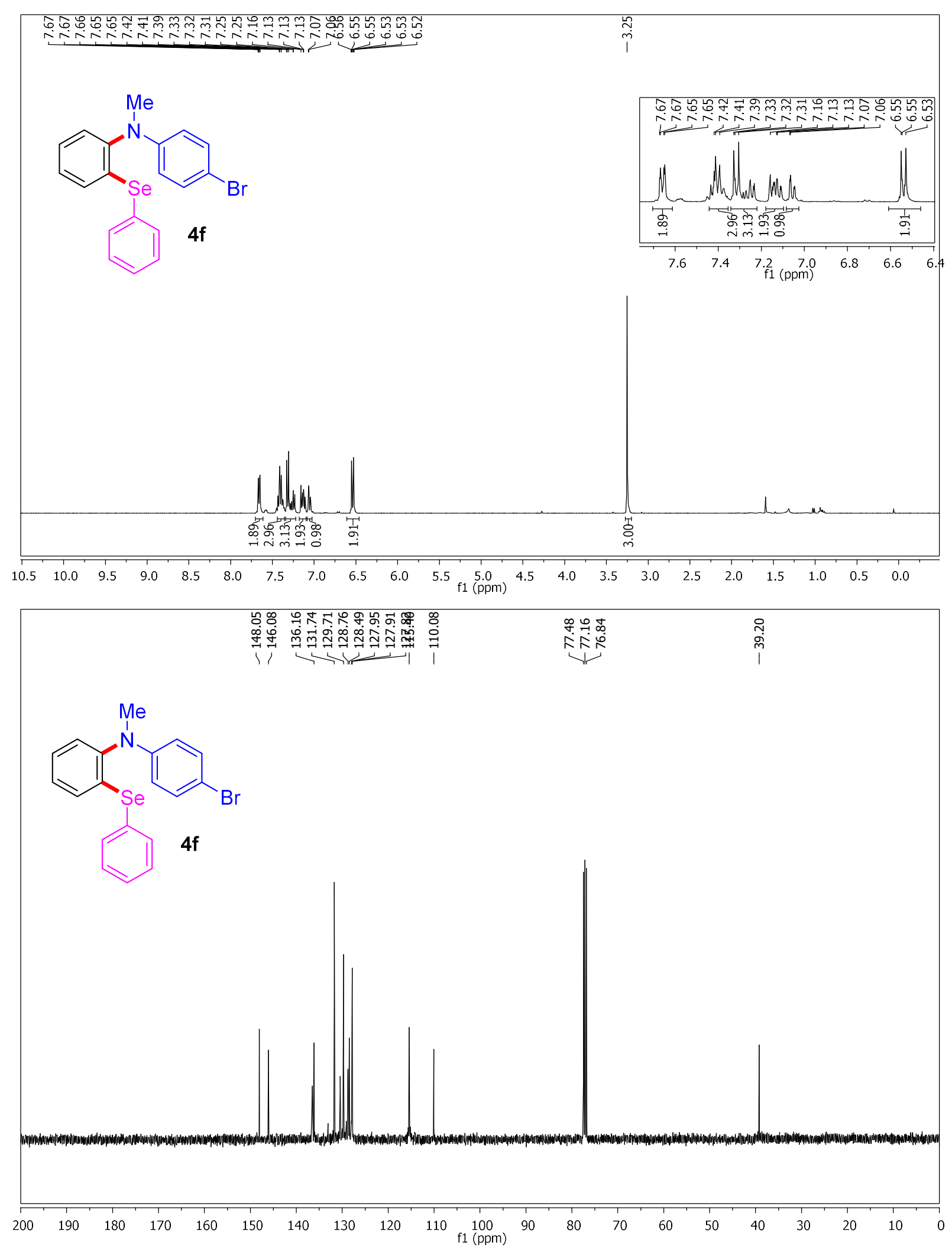

$\mathrm{N}$-(4-Chlorophenyl)- $\mathrm{N}$-methyl-2-(phenylselanyl)aniline (4g) 

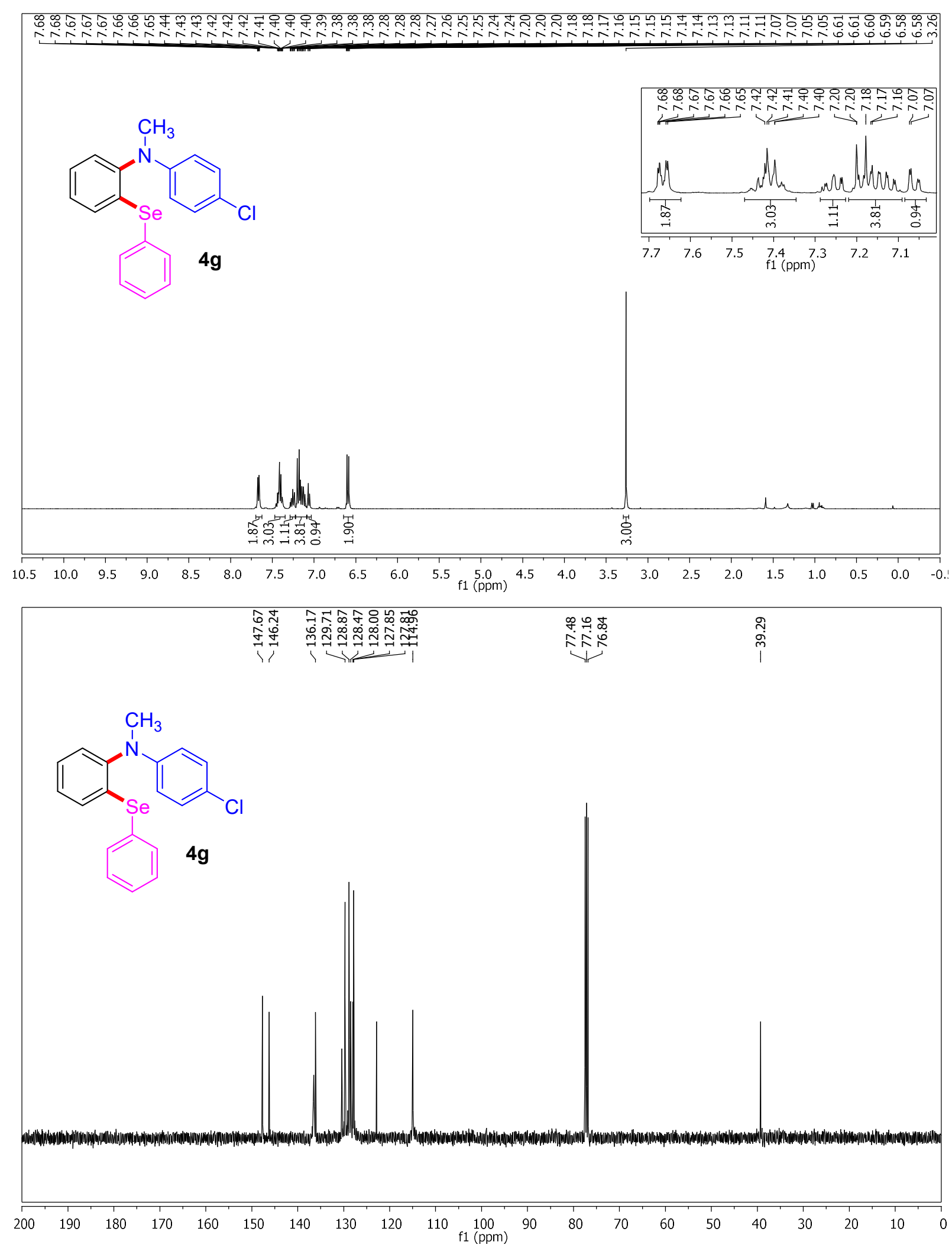

$\mathrm{N}$-(4-Fluorophenyl)- $\mathrm{N}$-methyl-2-(phenylselanyl)aniline (4h) 

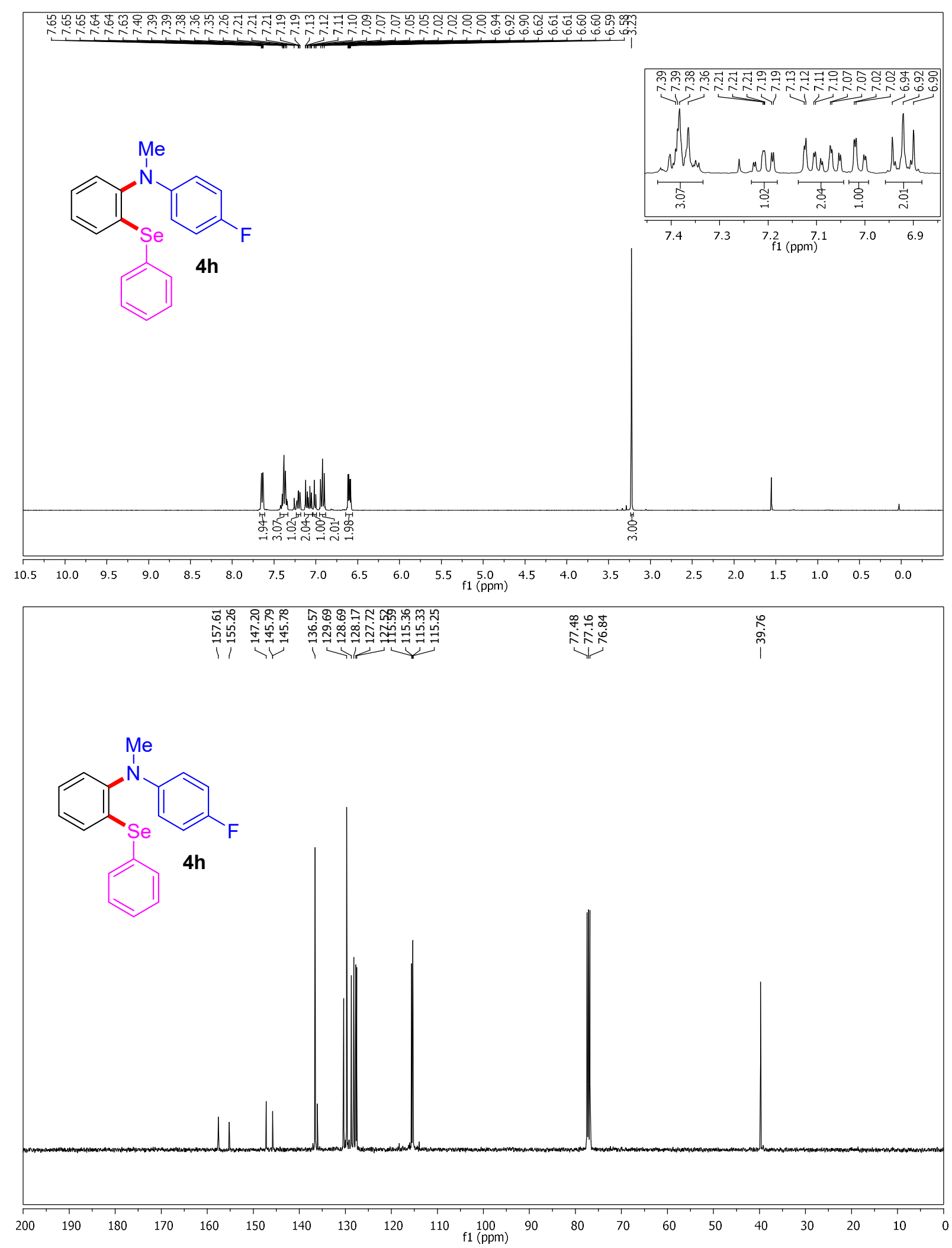

$\mathrm{N}$-Methyl-2-(phenylselanyl)- $\mathrm{N}$-(4-(trifluoromethyl)phenyl)aniline (4i) 

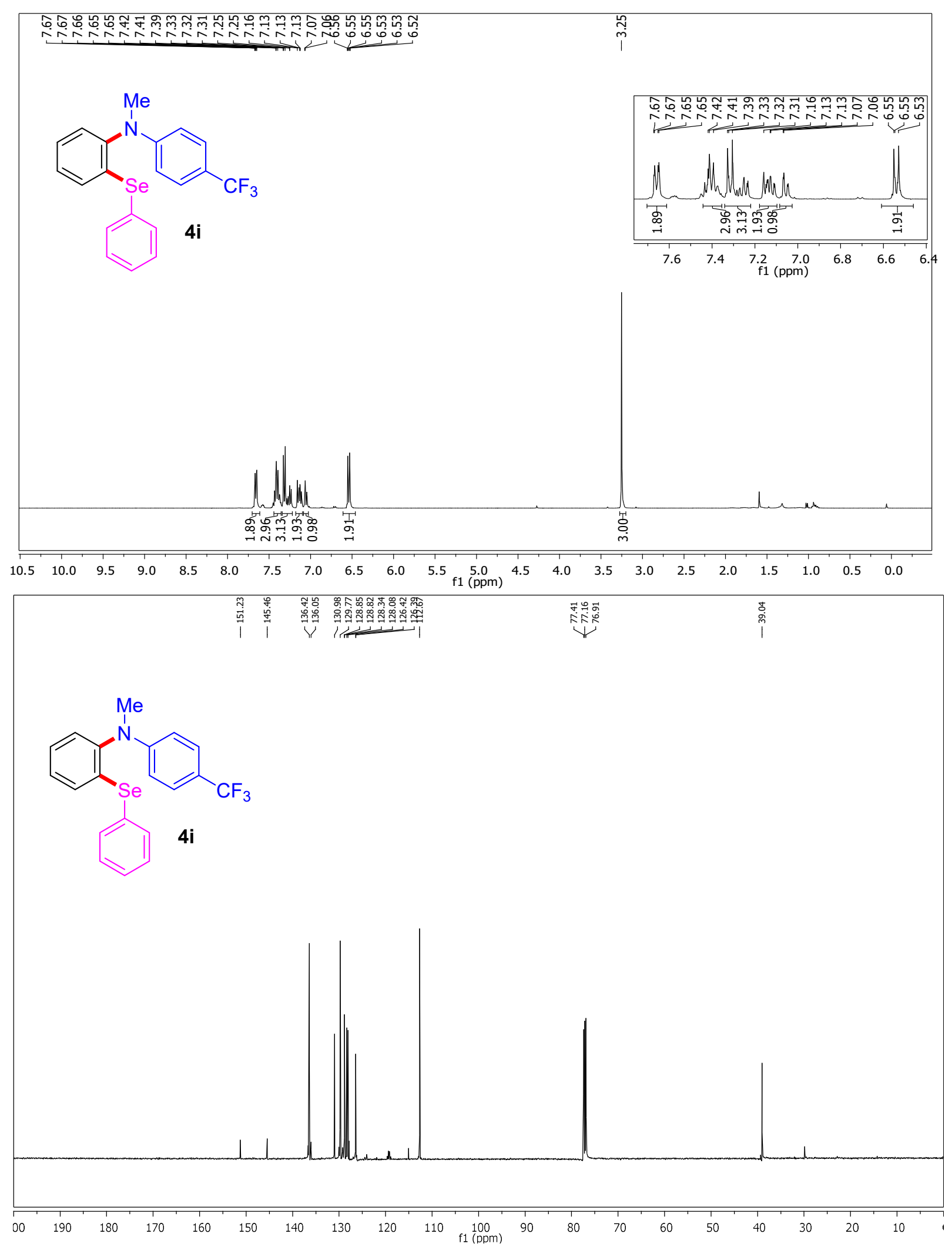

$\mathrm{N}$-Methyl-2-(phenylselanyl)- $\mathrm{N}$-(m-tolyl)aniline $(\mathbf{4 j})$ 

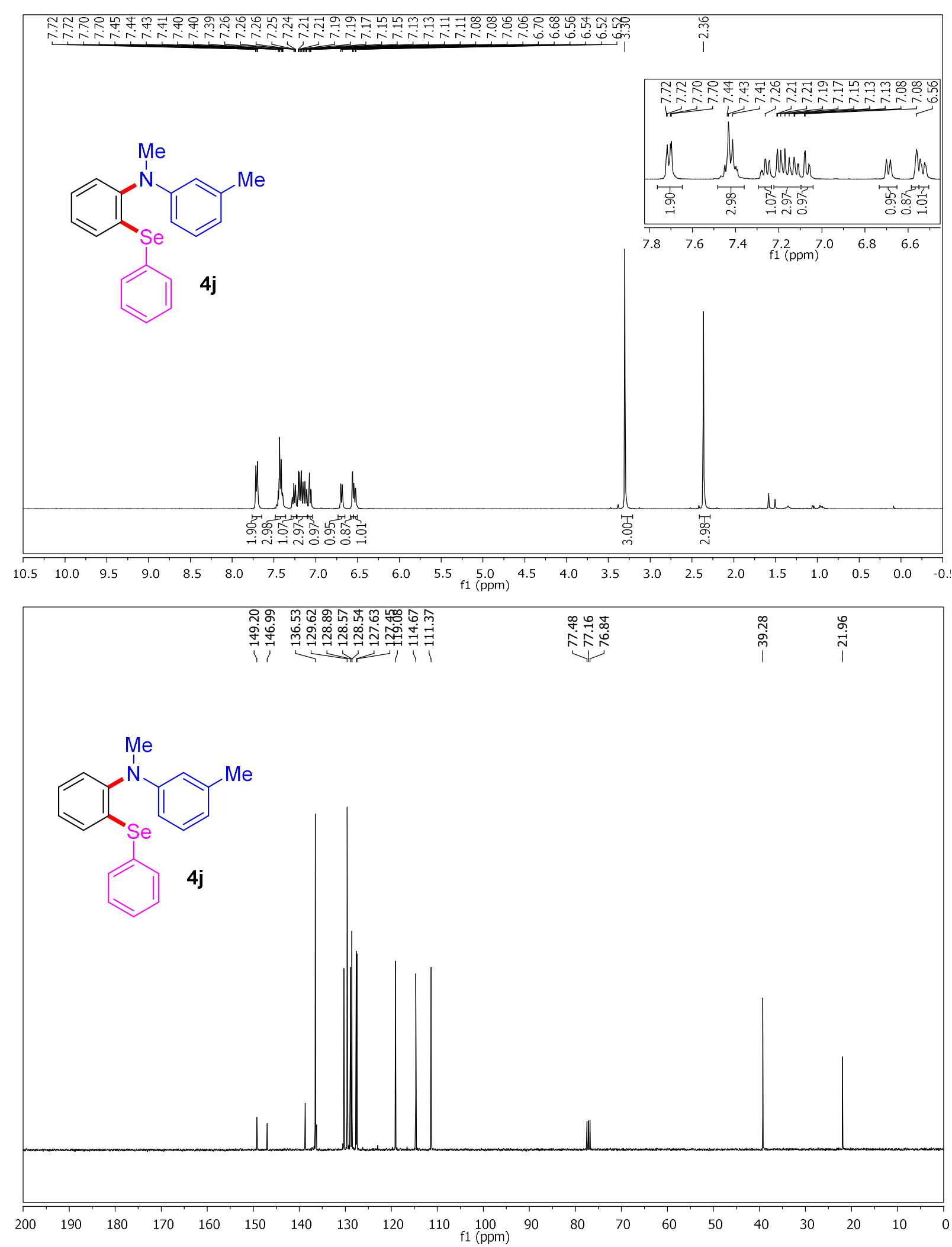
$\mathrm{N}$-(3-Chlorophenyl)- $\mathrm{N}$-methyl-2-(phenylselanyl)aniline (4k)

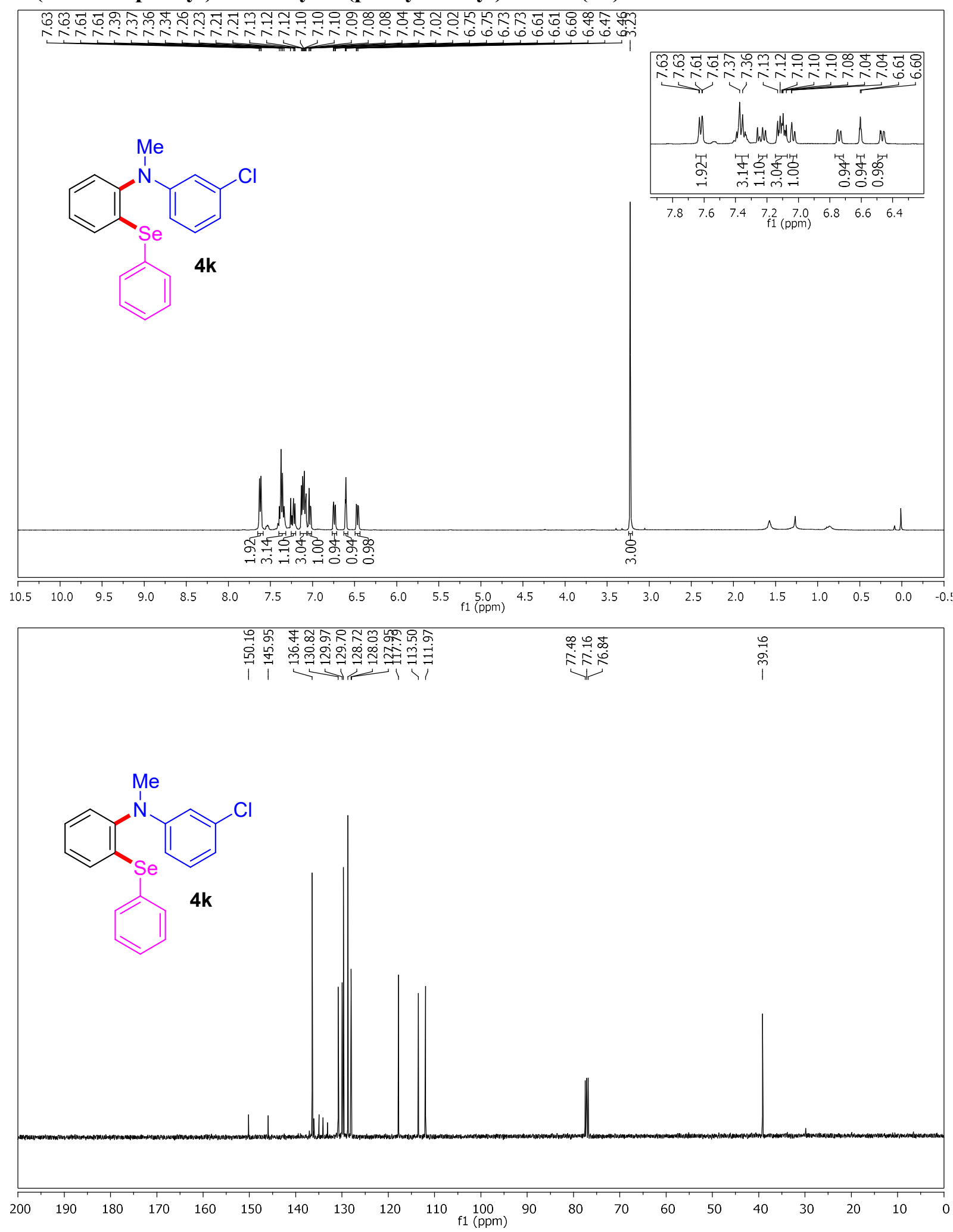

$N$-(3-Bromophenyl)- $N$-methyl-2-(phenylselanyl)aniline (4l) 

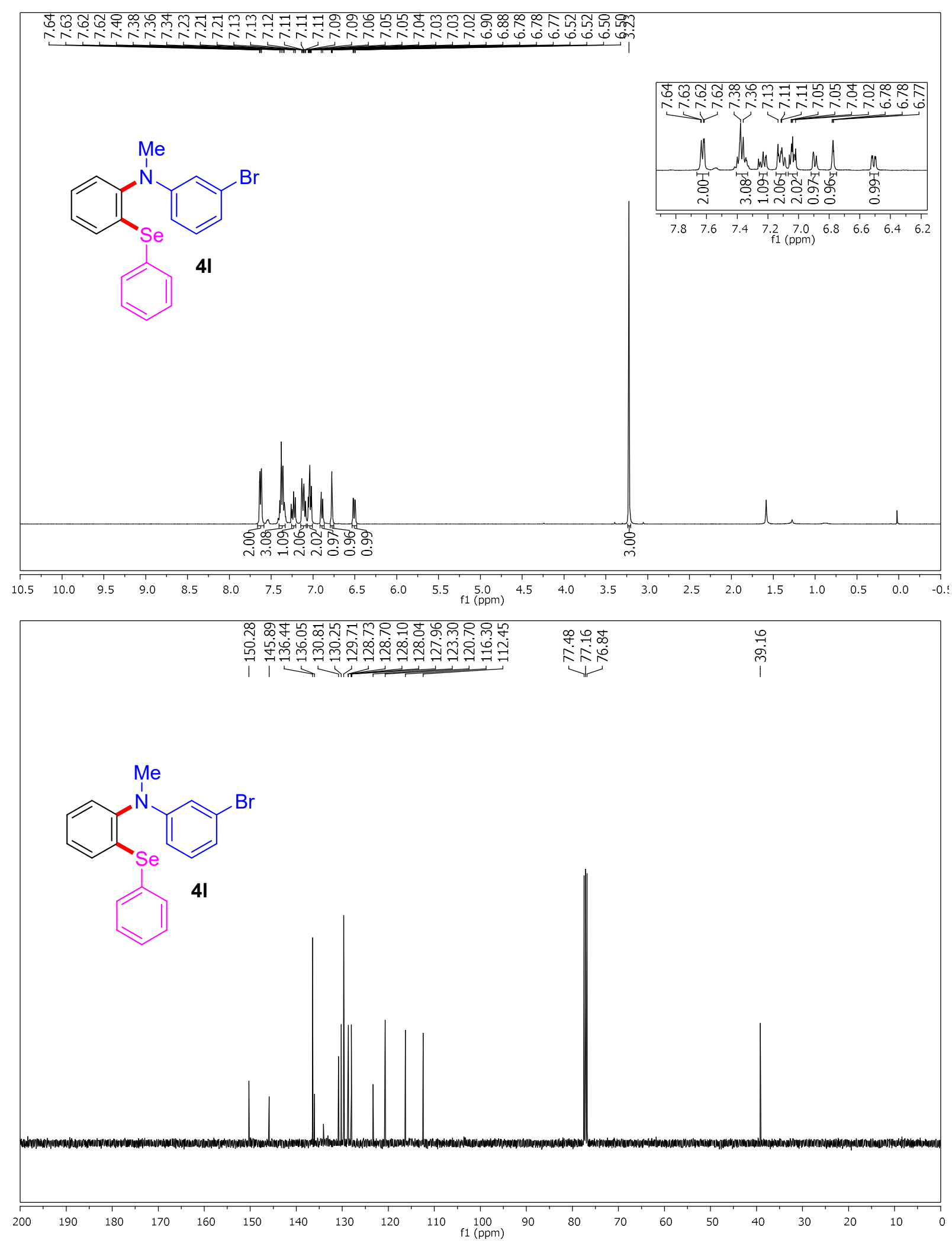
$\mathrm{N}$-Methyl-2-(phenylselanyl)- $\mathrm{N}$-(3-(trifluoromethyl)phenyl)aniline (4m)
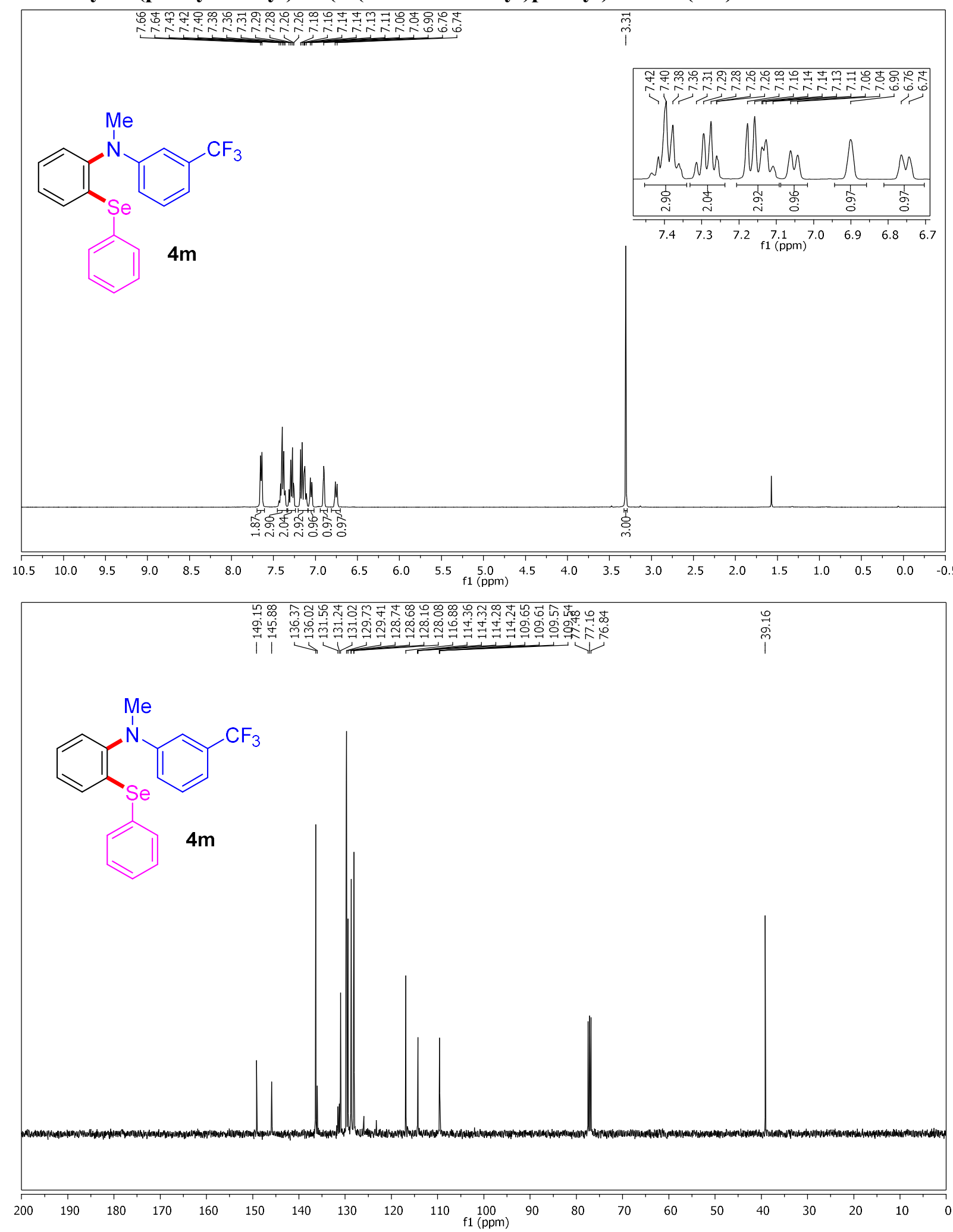

$N, 2-D i m e t h y l-N-(2-(p h e n y l s e l a n y l) p h e n y l) a n i l i n e(4 n)$ 

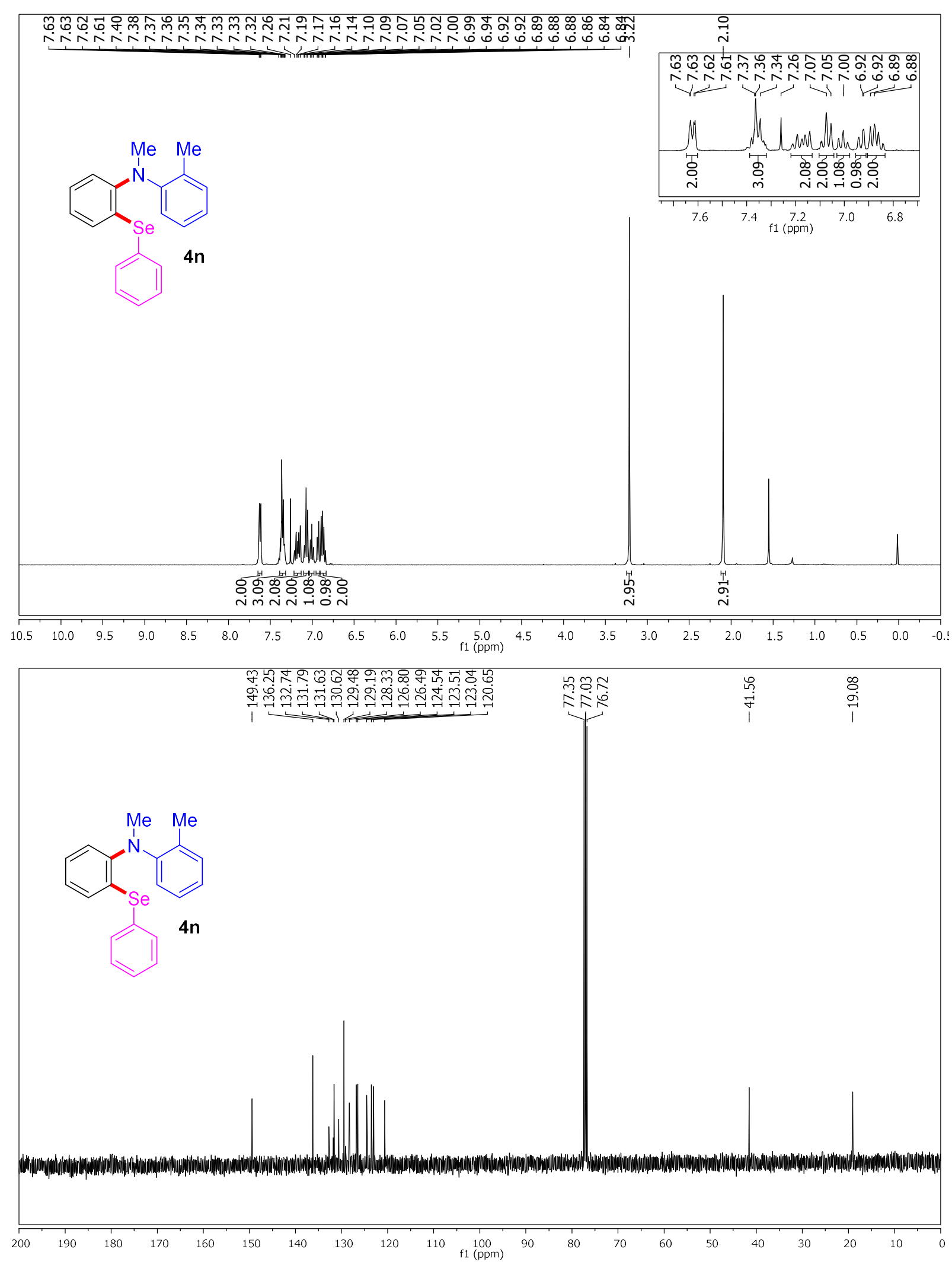

3,4-Difluoro- $N$-methyl- $N$-(2-(phenylselanyl)phenyl)aniline (4o) 

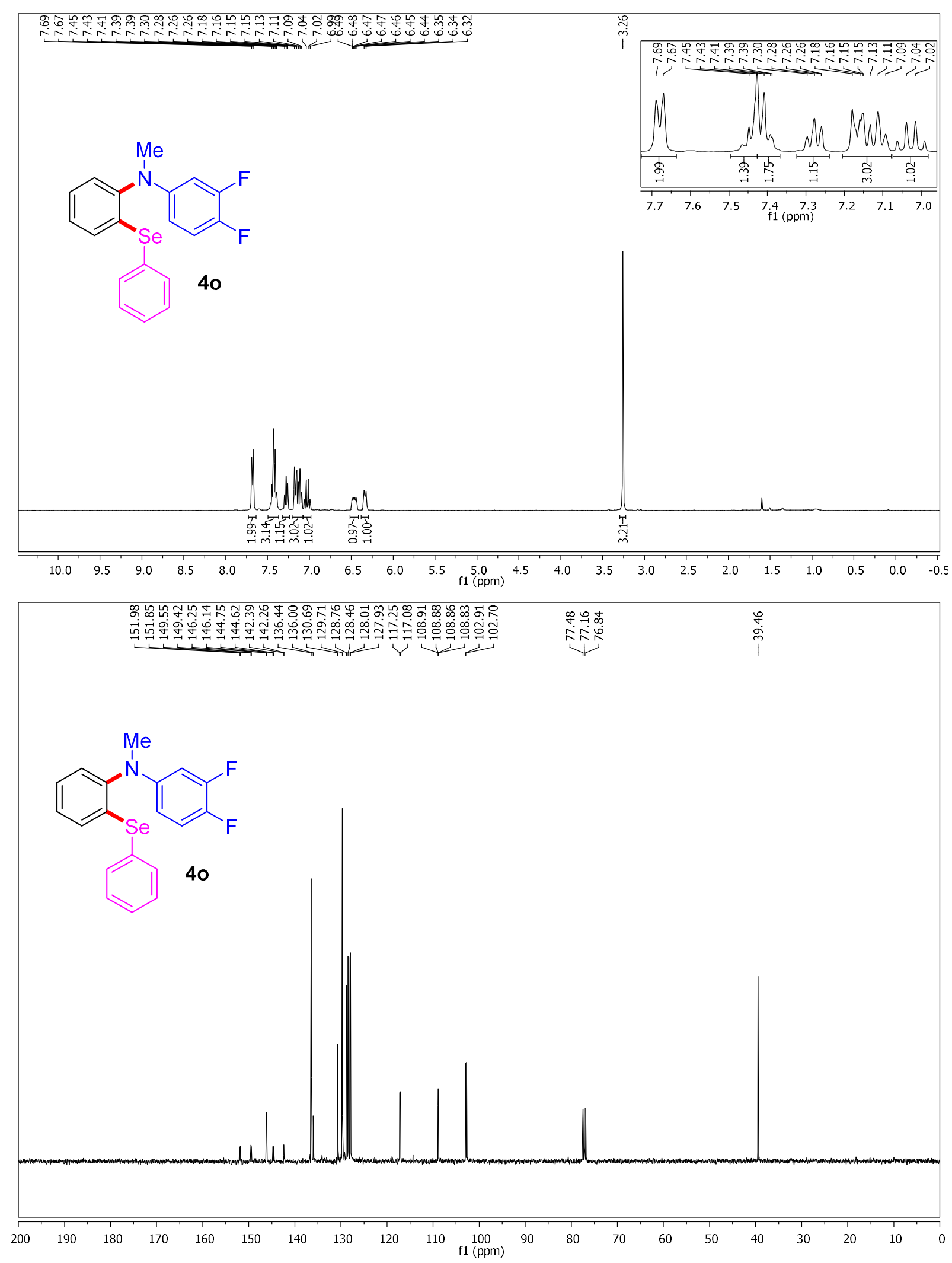

$\mathrm{N}$-Methyl- $\mathrm{N}$-(2-(phenylselanyl)phenyl)naphthalen-1-amine (4p) 

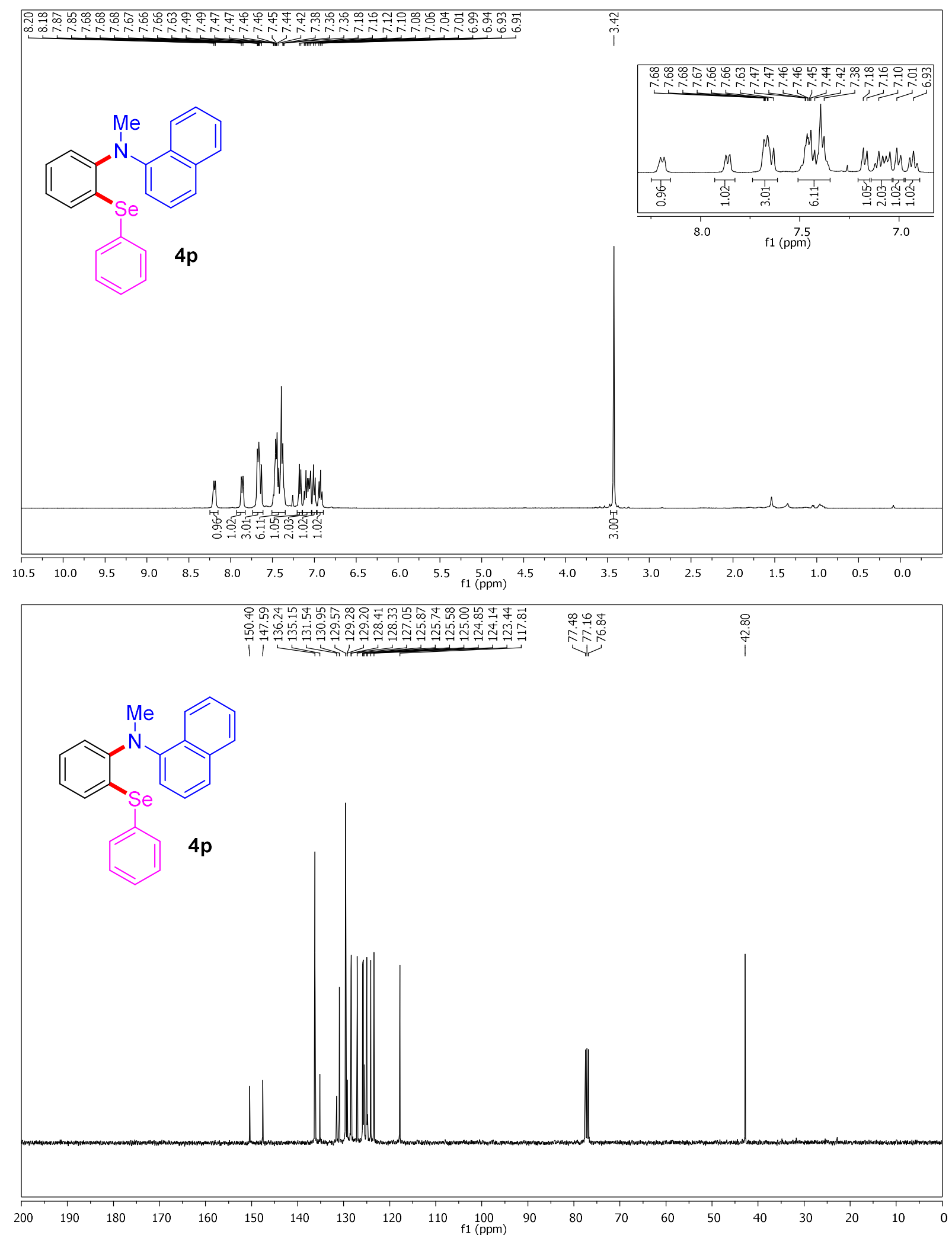

Ethyl (E)-3-(4-(methyl(2-(phenylselanyl)phenyl)amino)phenyl)acrylate (4q) 

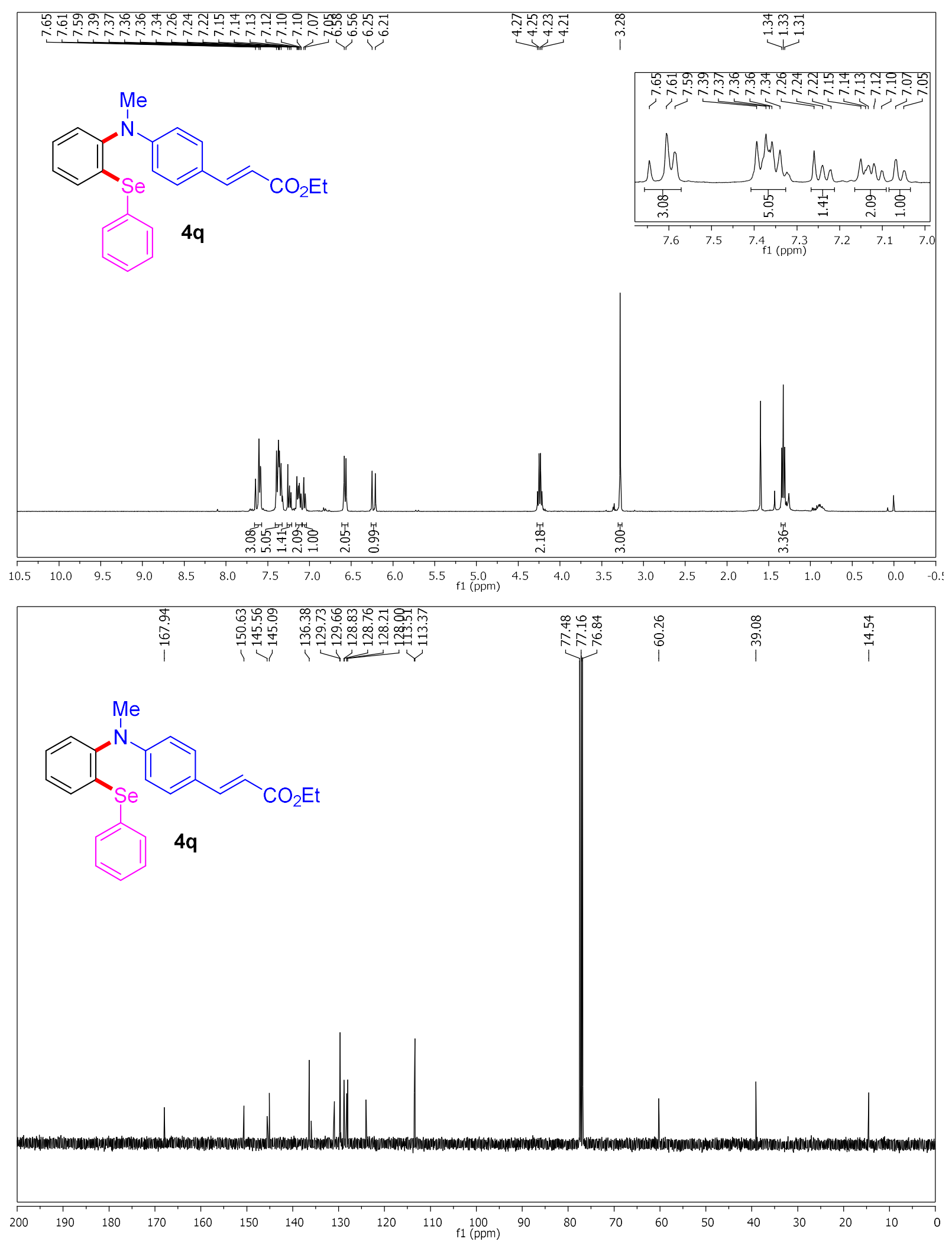

$N$-Methyl- $N$-phenyl-2-(p-tolylselanyl)aniline (4r)

S47 

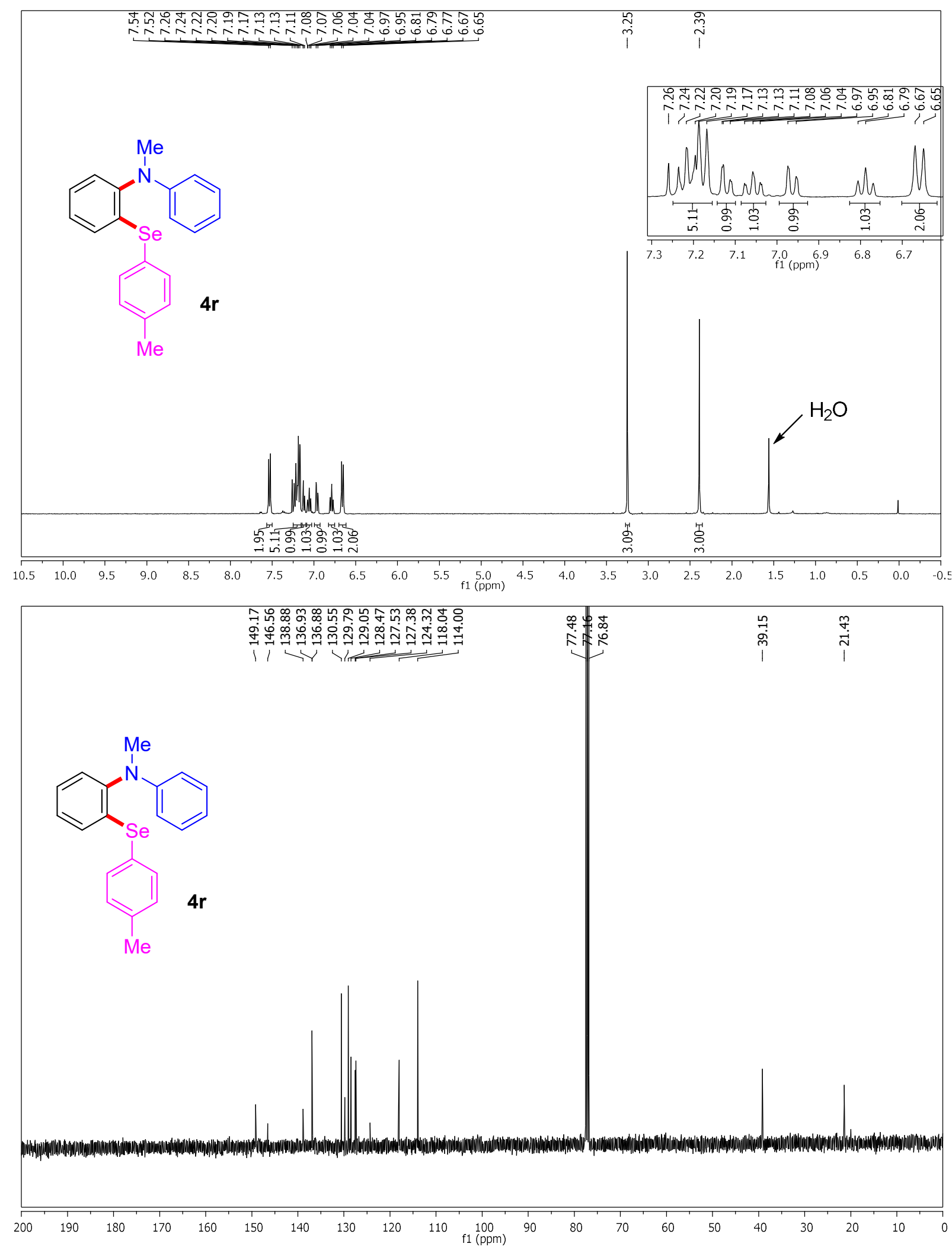

2-((4-Bromophenyl)selanyl)- $N$-methyl- $N$-phenylaniline (4s) 

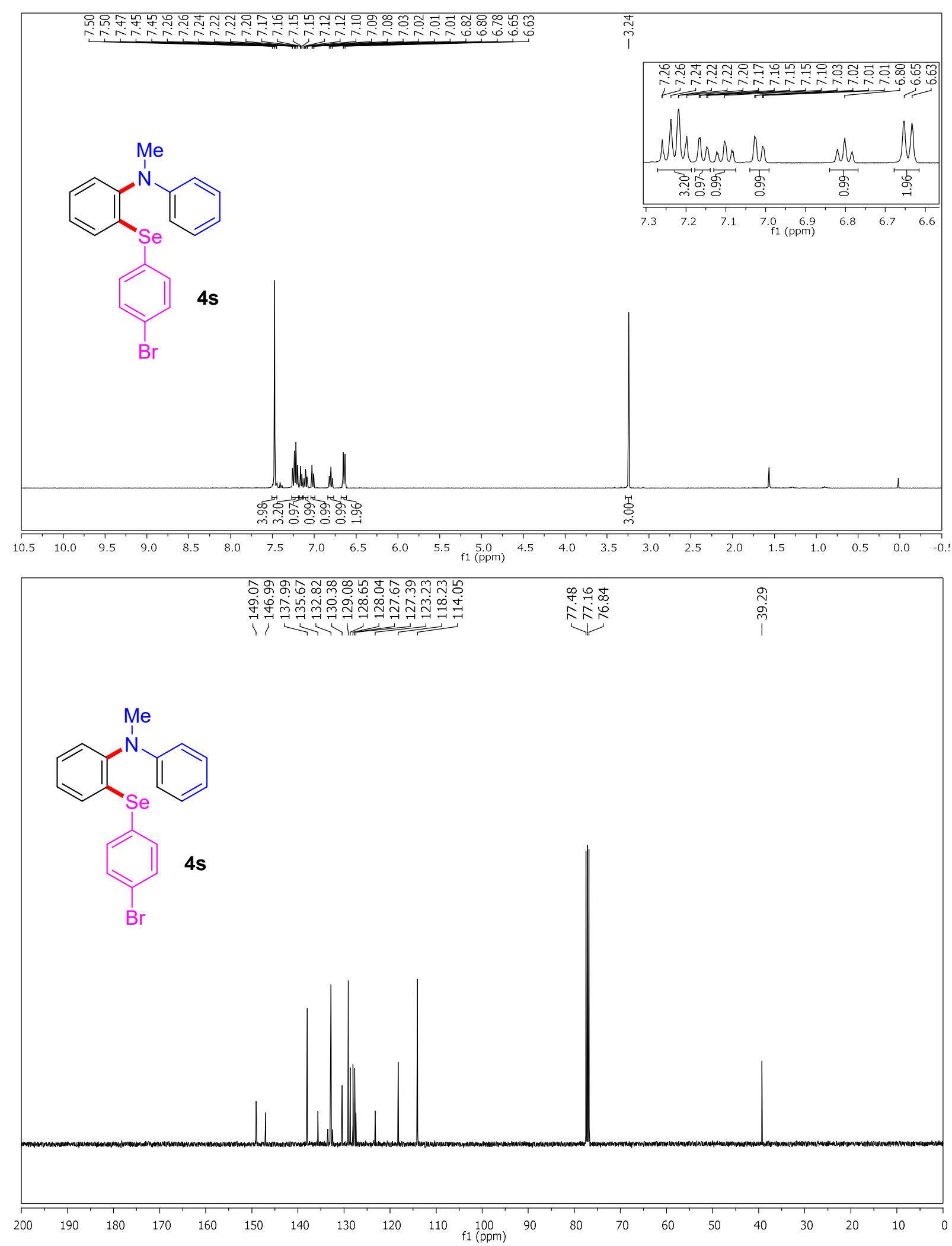

2-((4-Chlorophenyl)selanyl)- $N$-methyl- $N$-phenylaniline (4t) 

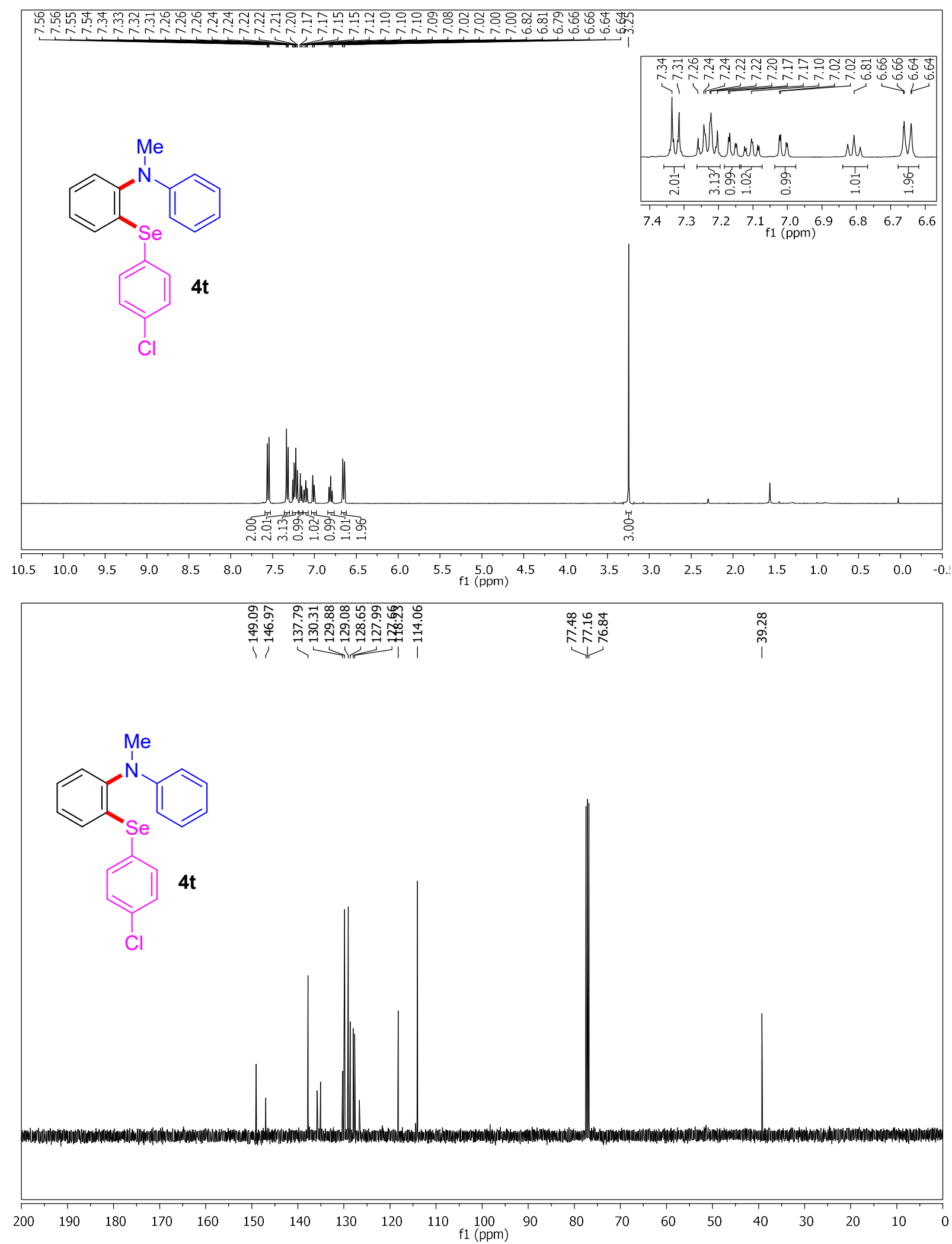

2-((4-Fluorophenyl)selanyl)- $N$-methyl- $N$-phenylaniline (4u) 

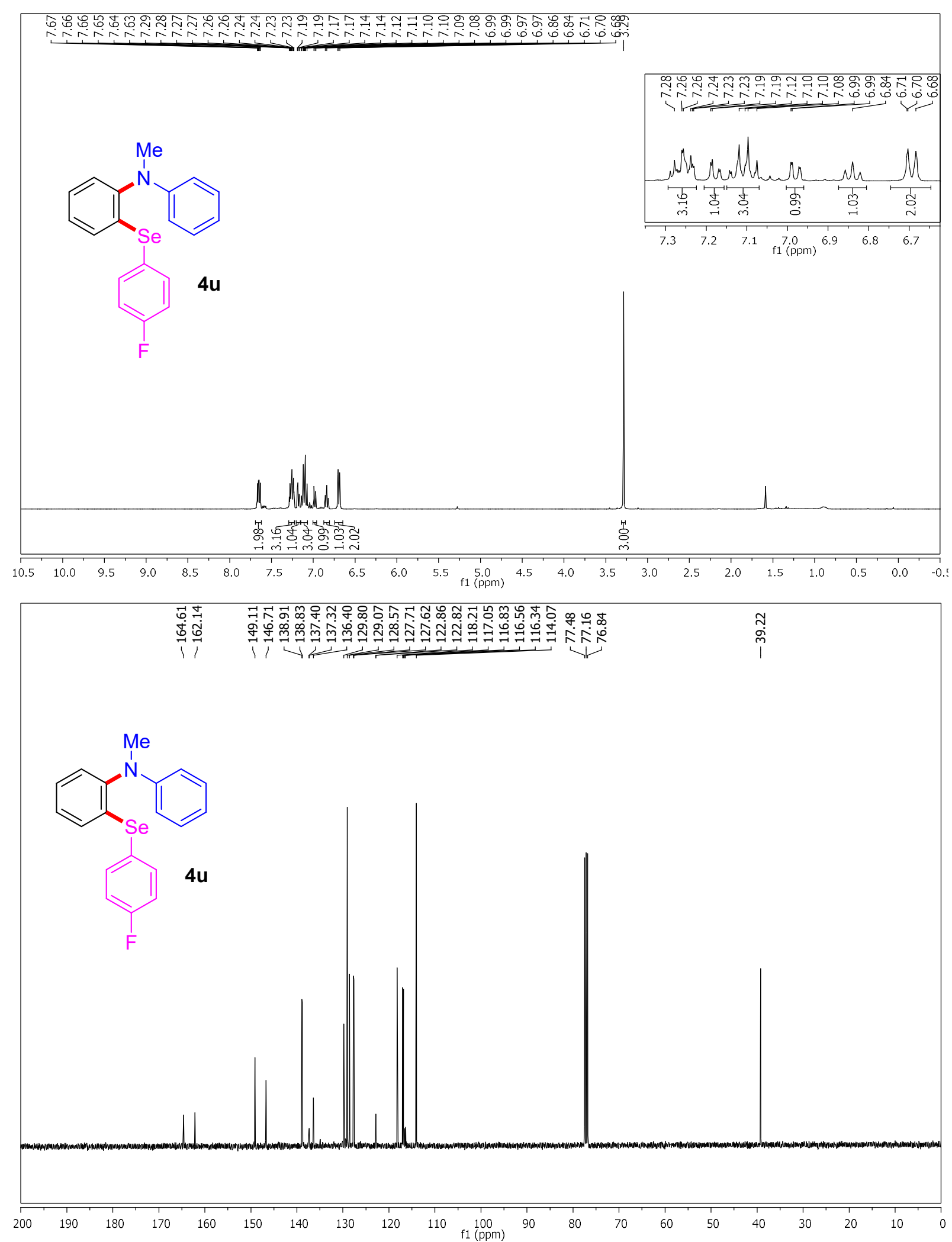

2-((3-Chlorophenyl)selanyl)- $N$-methyl- $N$-phenylaniline (4v) 

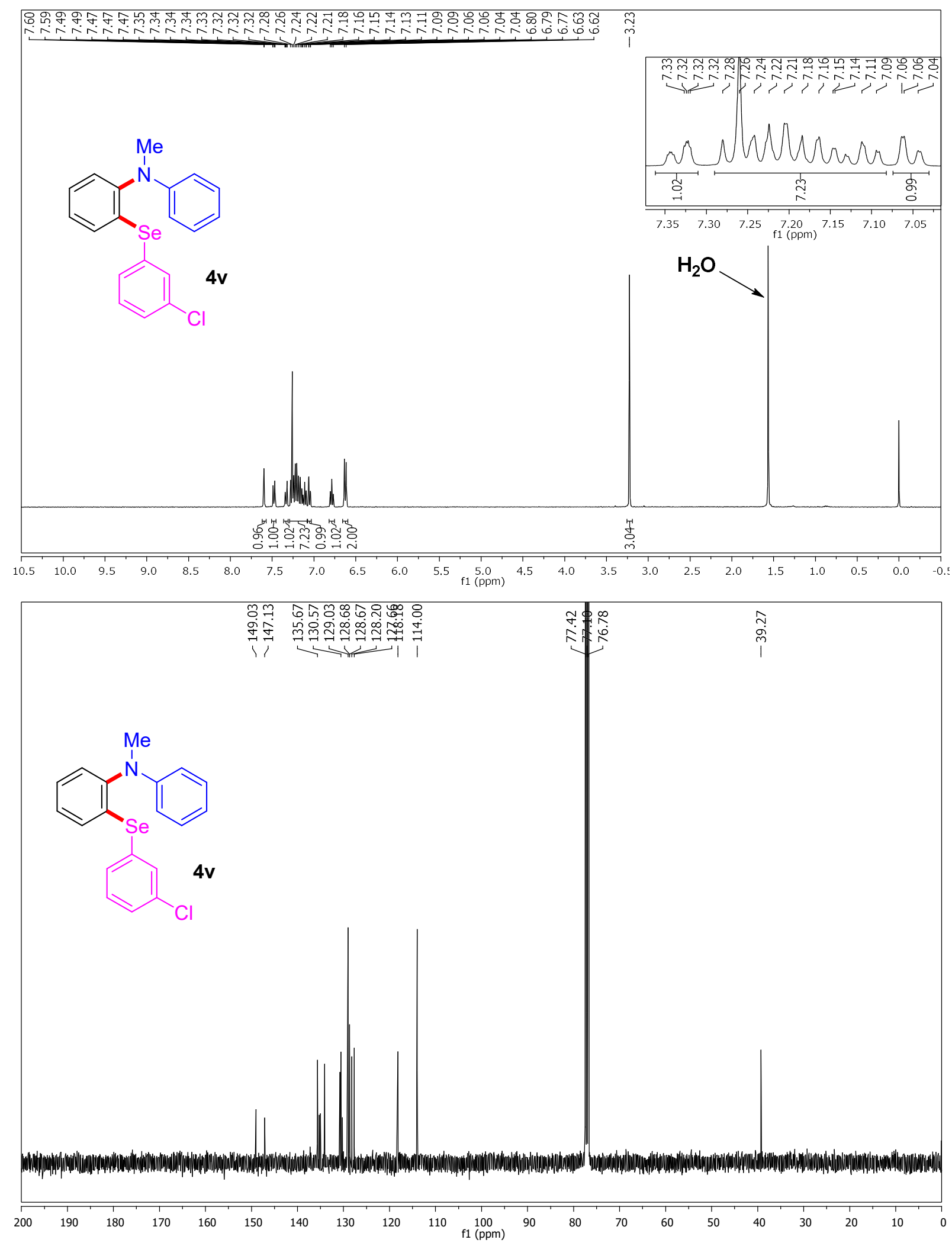

2-((2-Chlorophenyl)selanyl)- $N$-methyl- $N$-phenylaniline (4w) 

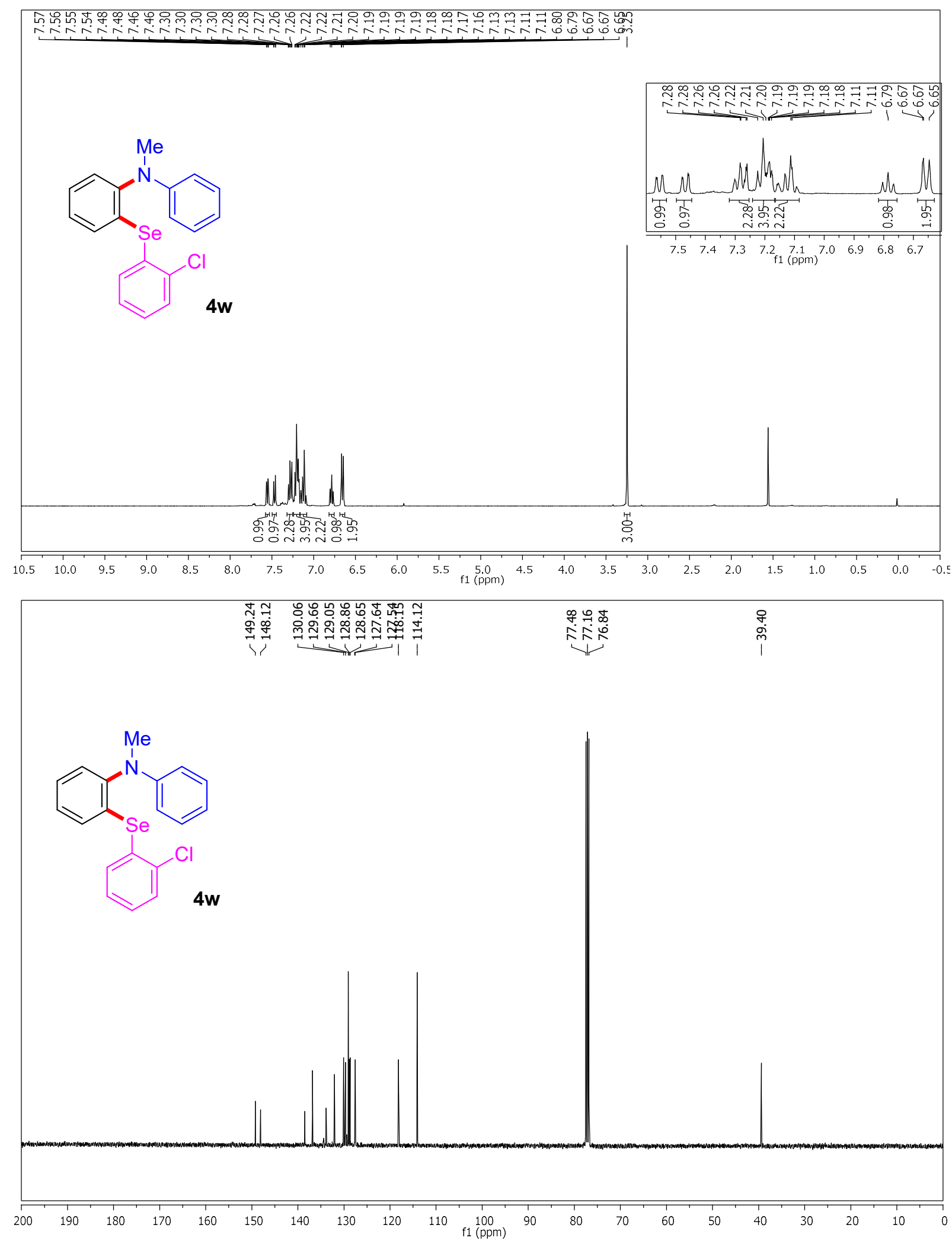

$N, 4,5-T$ rimethyl- $N$-phenyl-2-(phenylselanyl)aniline (4x) 

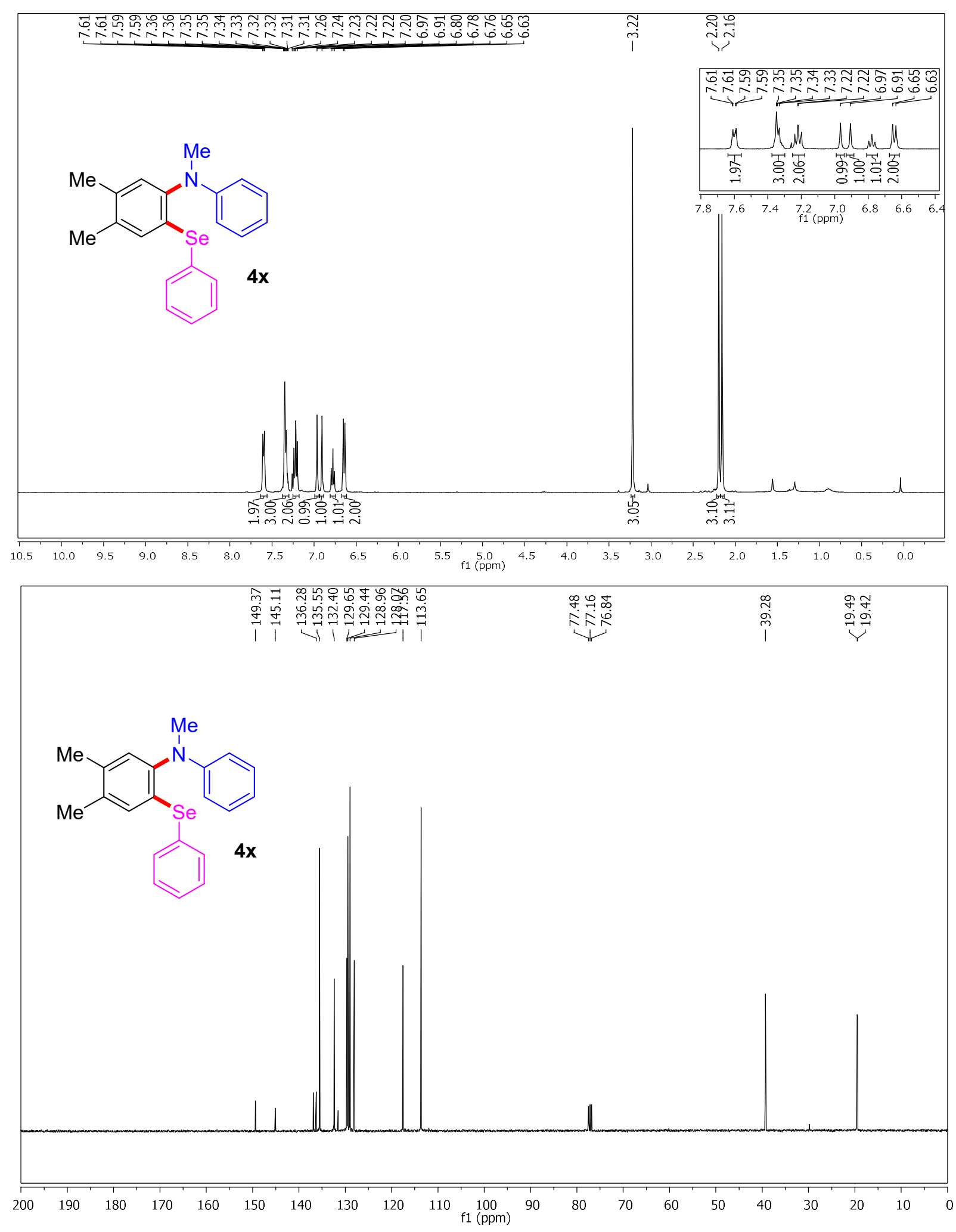

$\mathrm{N}$-Methyl- $\mathrm{N}$-phenyl-6-(phenylselanyl)-2,3-dihydro-1 $\mathrm{H}$-inden-5-amine (4y) 

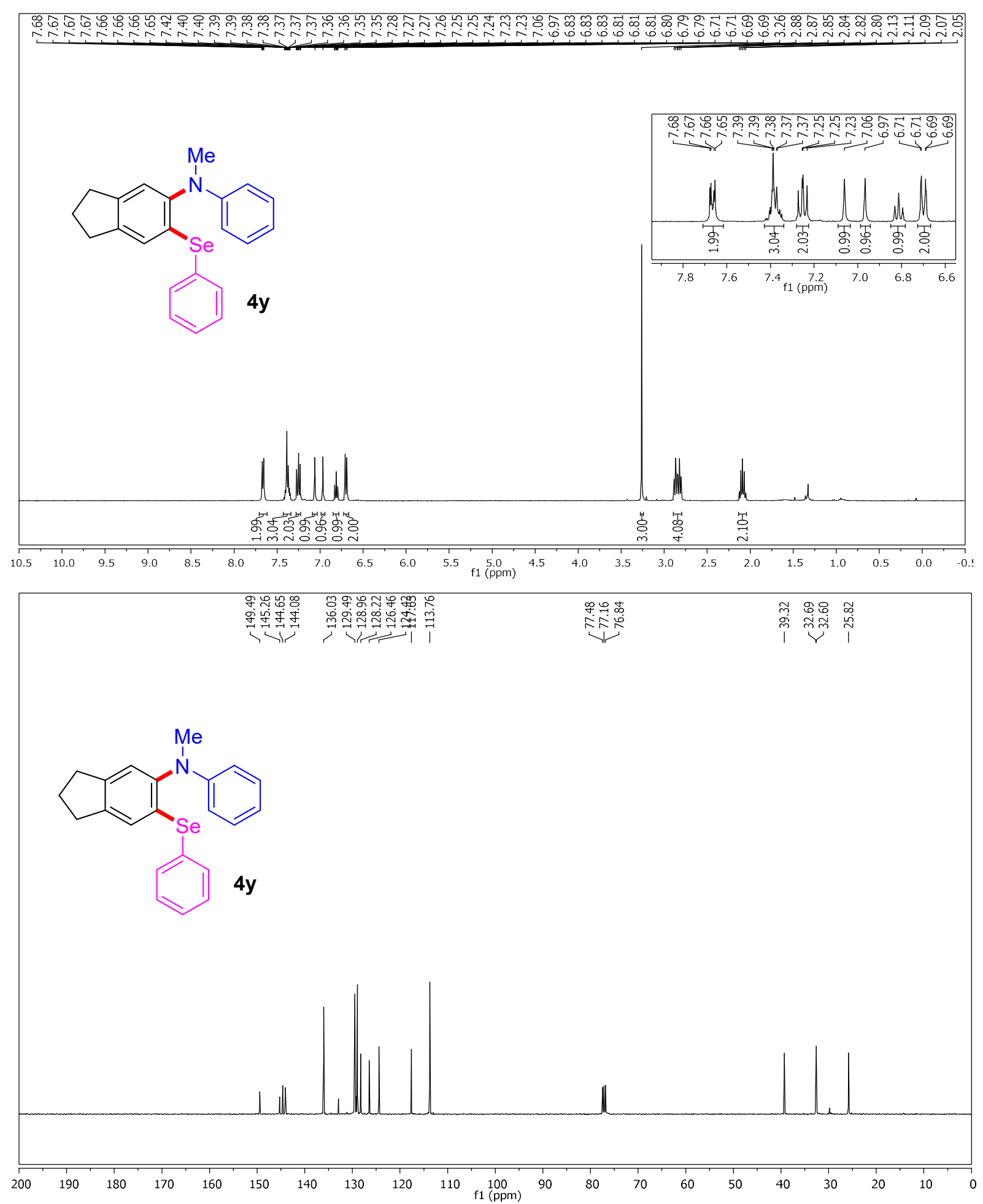

4,5-Difluoro- $N$-methyl- $N$-phenyl-2-(phenylselanyl)aniline (4z) 

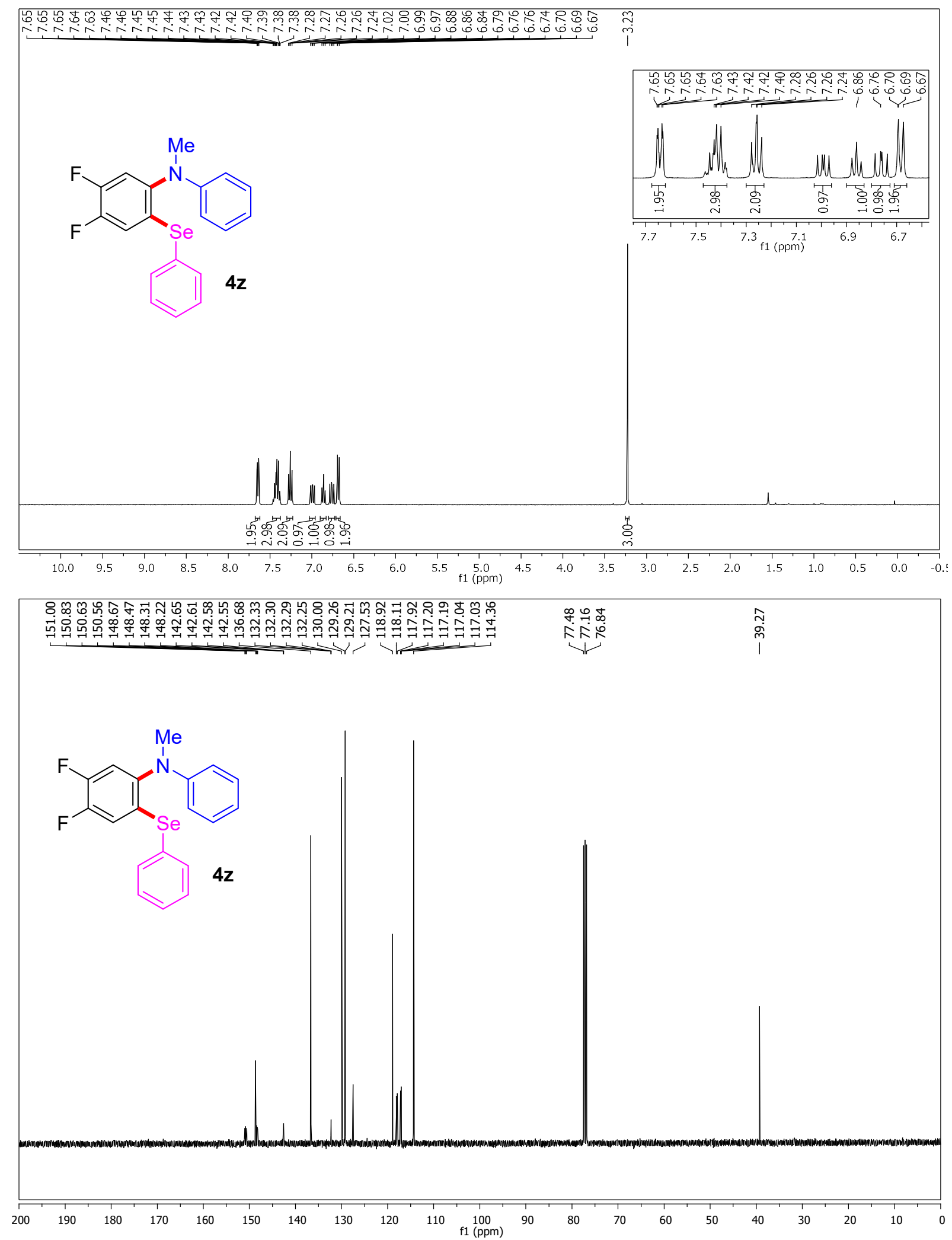

$\mathrm{N}$-Methyl- $N$-phenyl-1-(phenylselanyl)naphthalen-2-amine (4aa) 

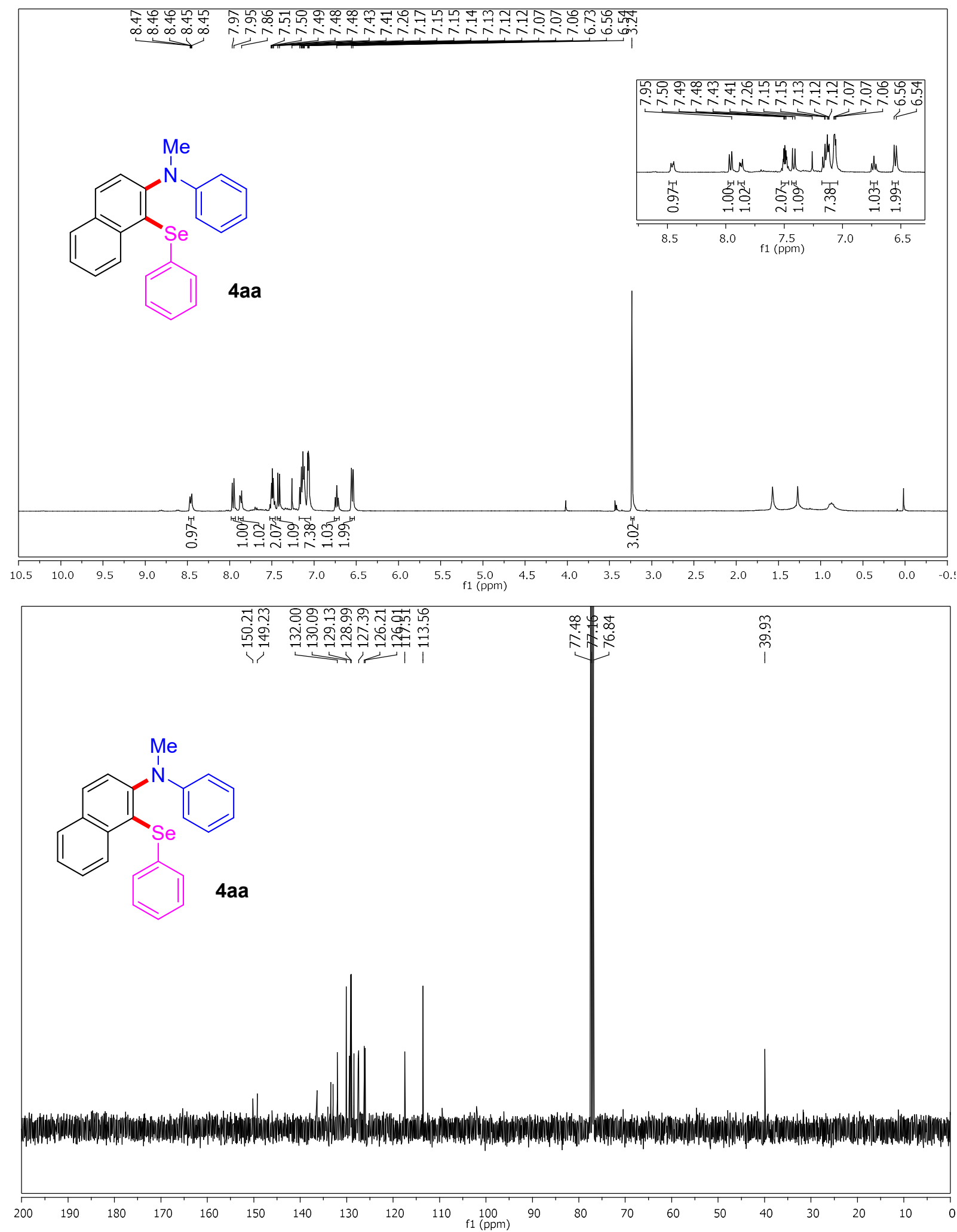

$N$-Methyl- $N$-phenyl-1-(phenylselanyl)-5,6,7,8-tetrahydronaphthalen-2-amine (4ab) 

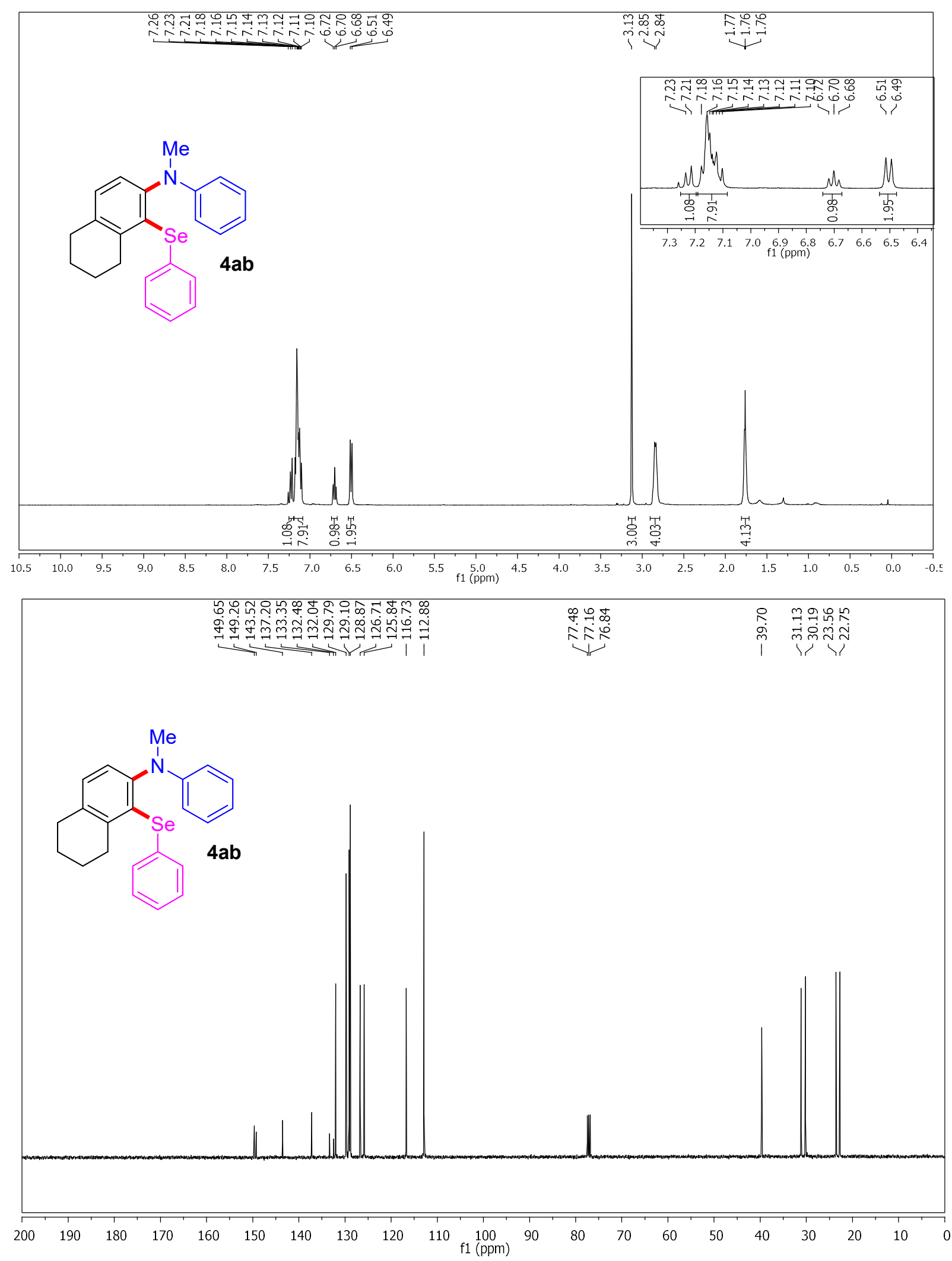

$N, 4-d i m e t h y l-N$-phenyl-2-(phenylselanyl)aniline (4ac) and 
$\boldsymbol{N}, 5$-dimethyl- $N$-phenyl-2-(phenylselanyl)aniline (4ac')
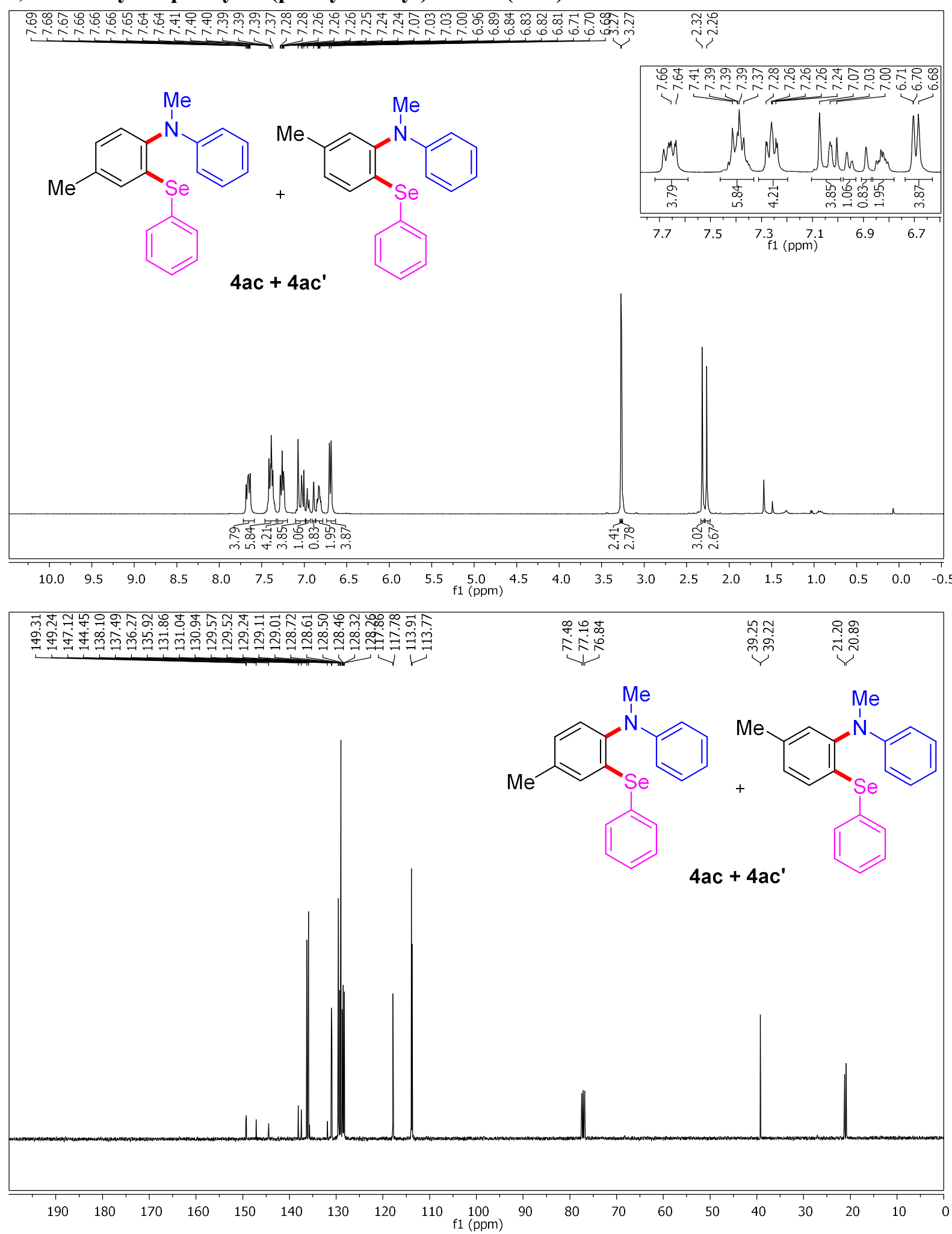

\section{4-Chloro- $N$-methyl- $N$-phenyl-2-(phenylselanyl)aniline (4ad) and}



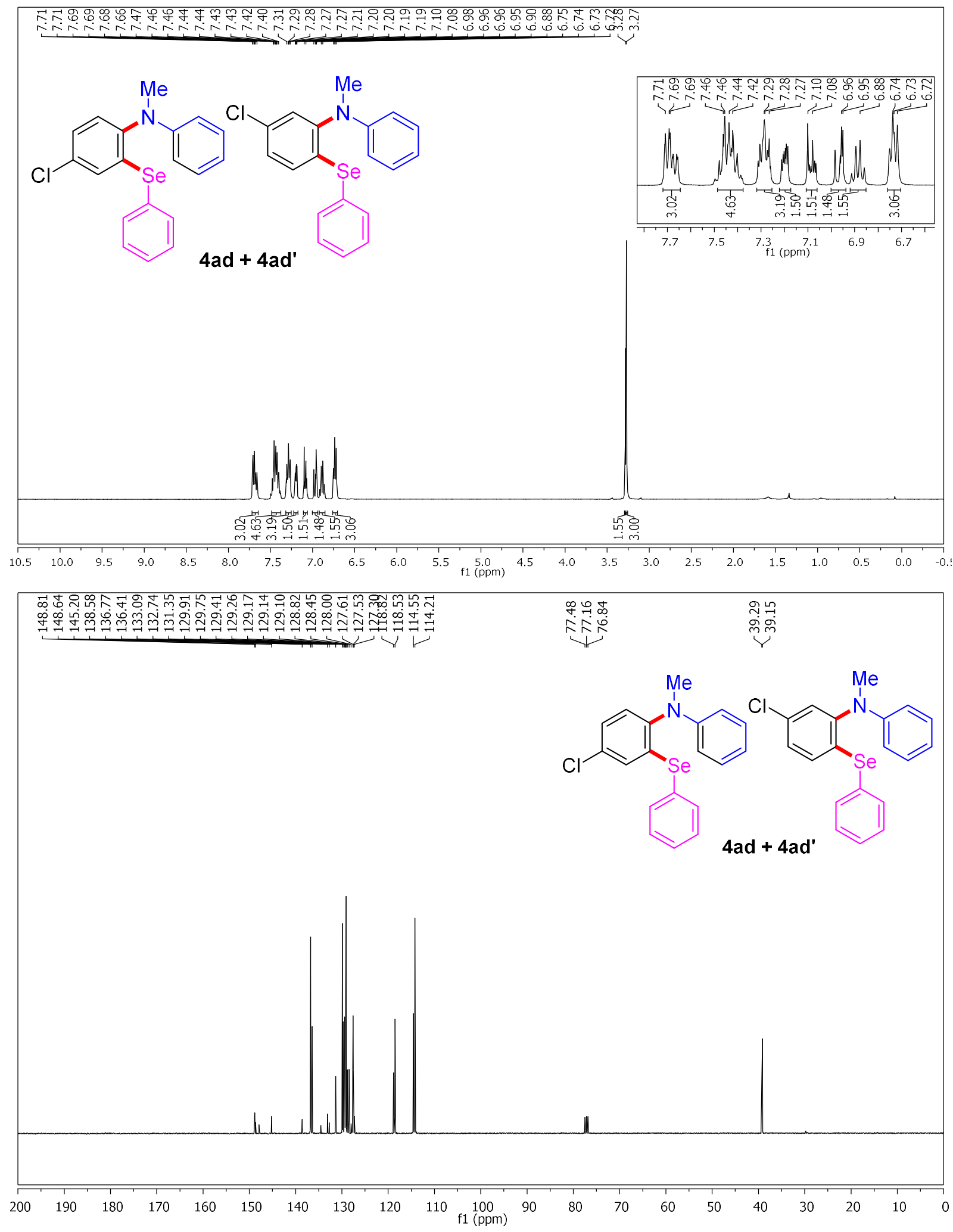

4-Fluoro- $N$-methyl- $N$-phenyl-2-(phenylselanyl)aniline (4ae) and 


\section{5-fluoro- $N$-methyl- $N$-phenyl-2-(phenylselanyl)aniline (4ae')}
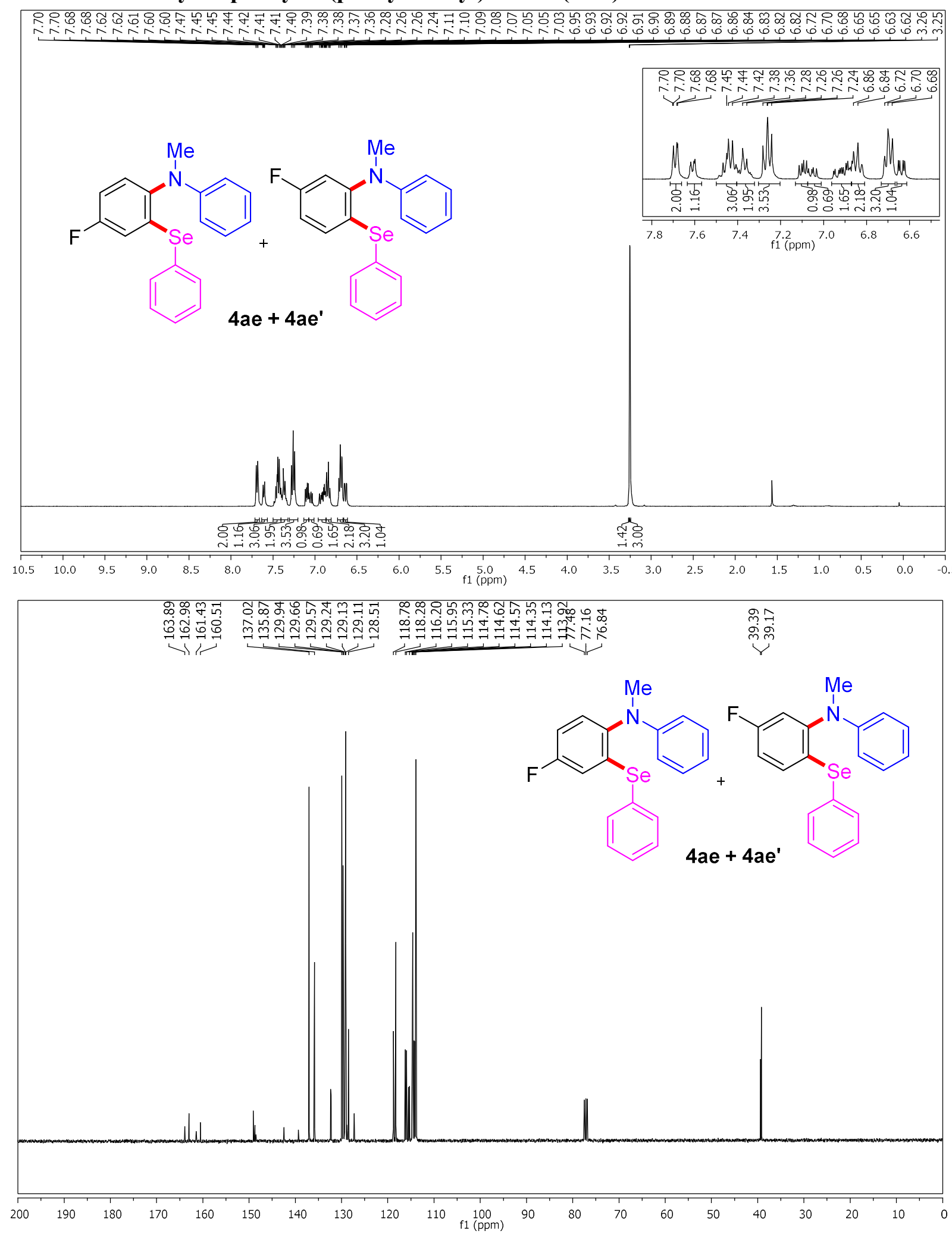

$N, N$-Dimethyl-2-(phenylselanyl)aniline (4af) 

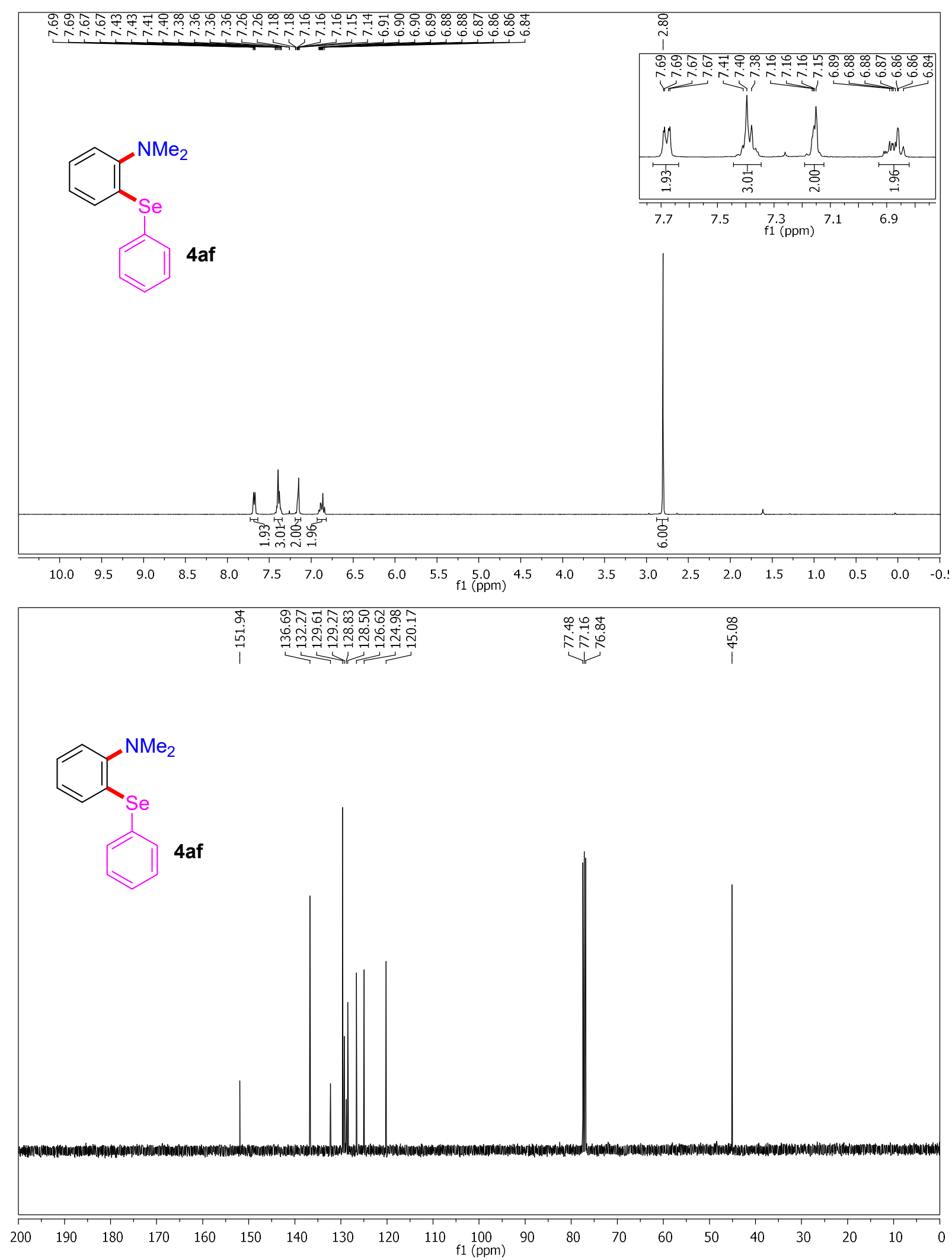

1-(2-(Phenylselanyl)phenyl)-1,2,3,4-tetrahydroquinoline (4ag) 


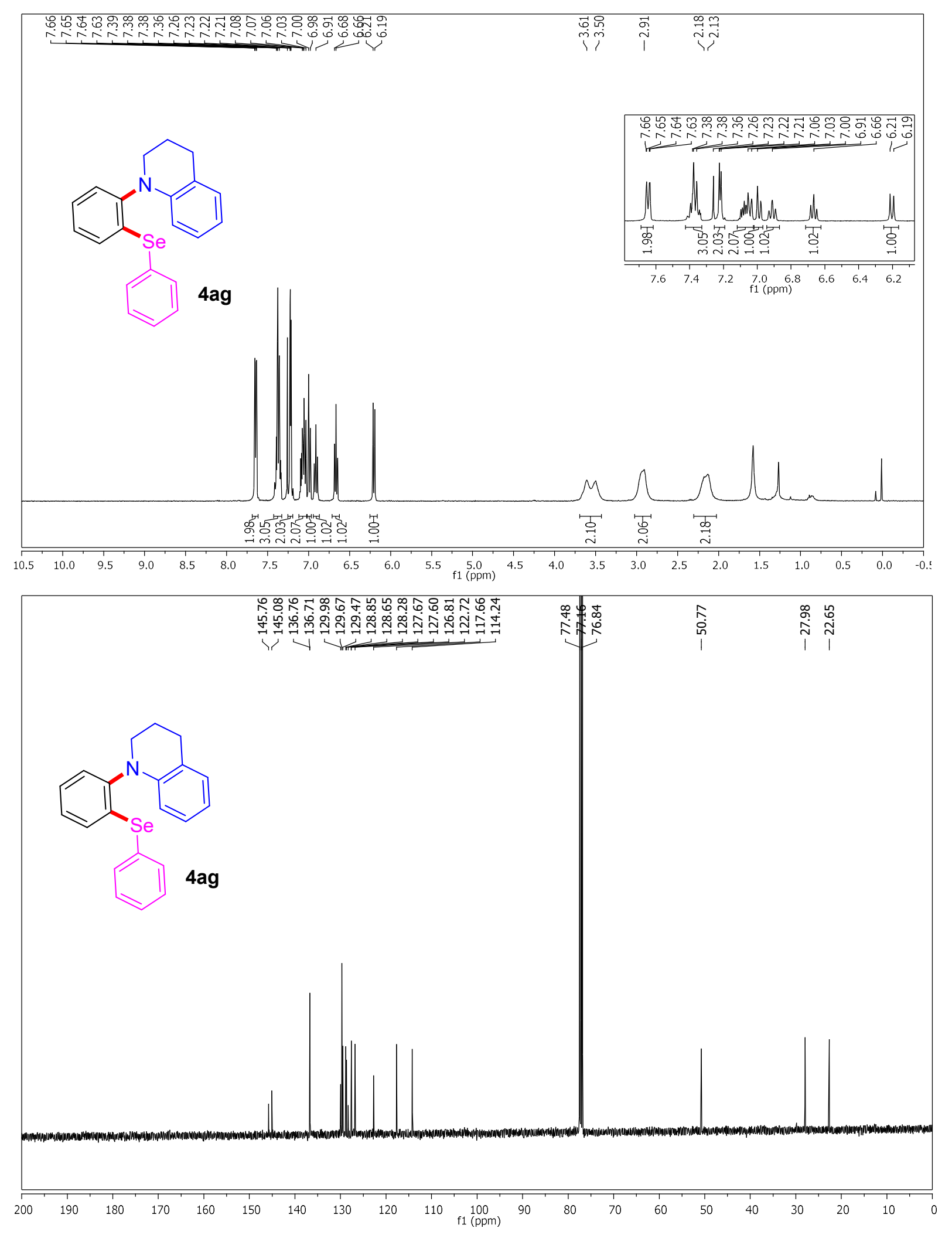


$\boldsymbol{N}$-Methyl- $\boldsymbol{N}$-phenyl-2-(phenylthio)aniline (9a)
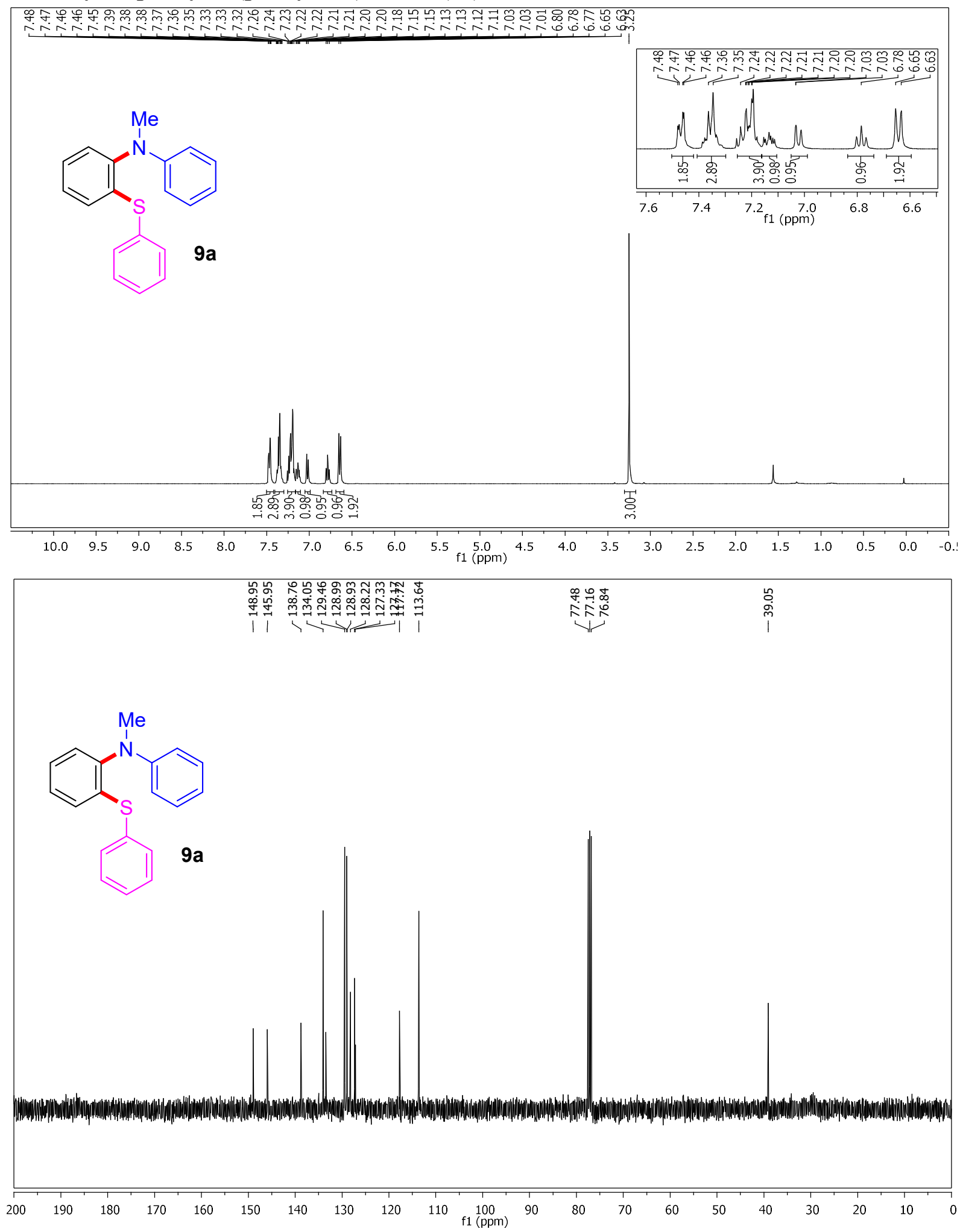


\section{$\mathrm{N}$-(4-Bromobutyl)- $\mathrm{N}$-phenyl-2-(phenylselanyl)aniline (13)}

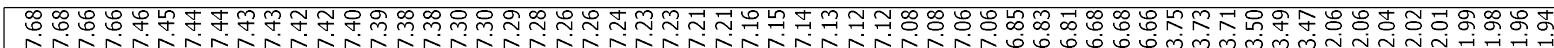
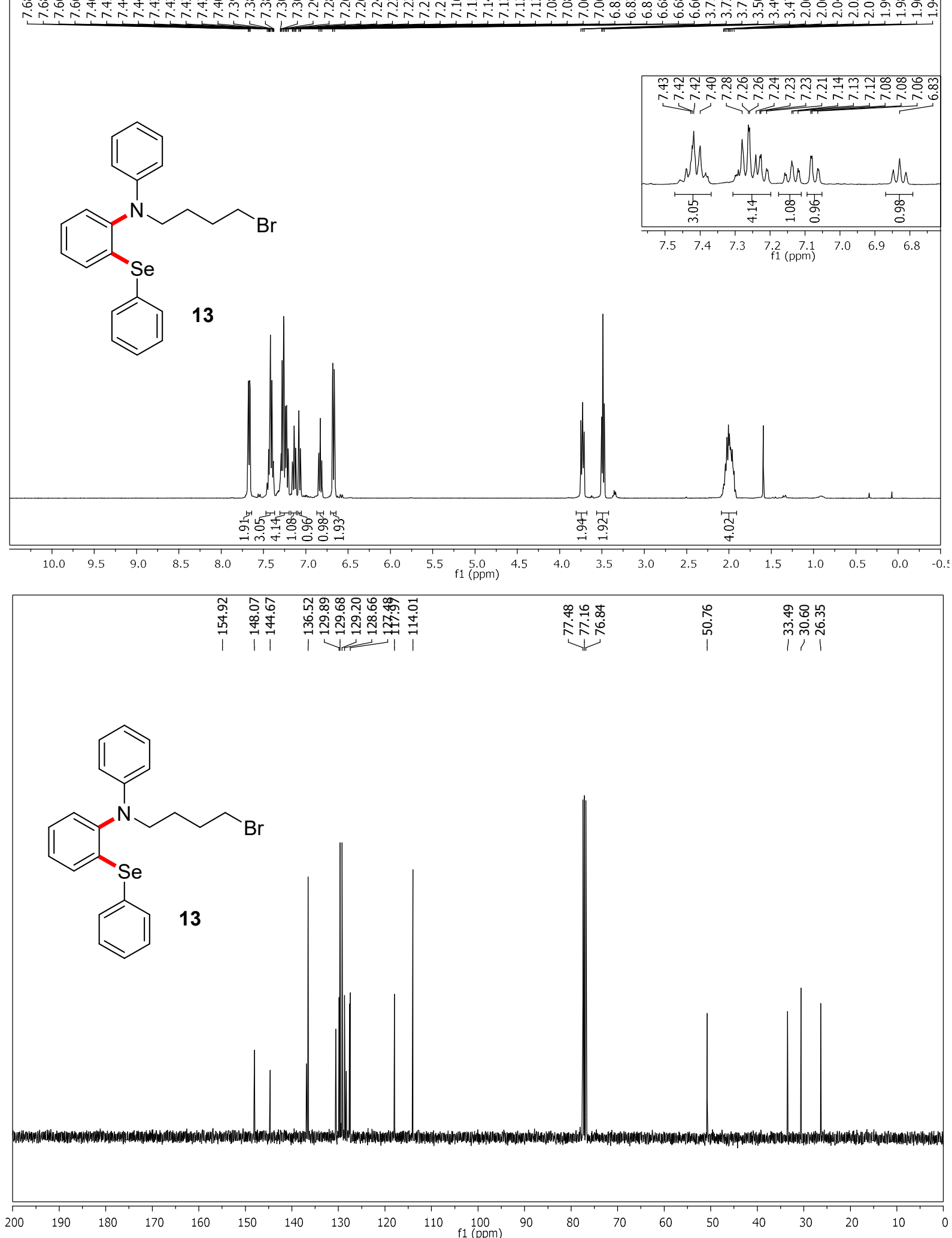


\section{$\mathrm{N}$-Phenyl-2-(phenylselanyl)- $\mathrm{N}$-(4-(phenylselanyl)butyl)aniline (14)}
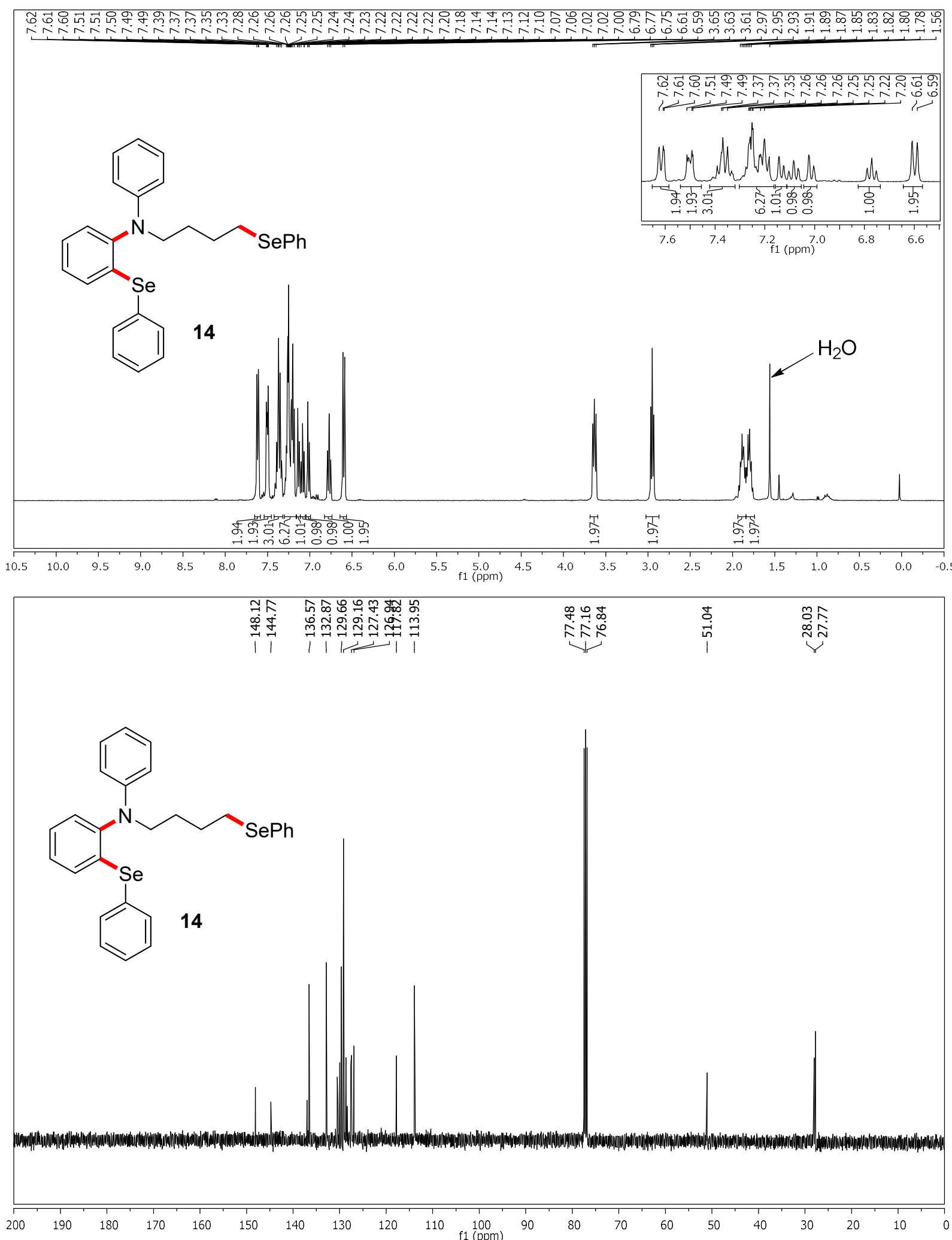